\title{
Blood, bones and brains : peripheral biological endophenotypes and their structural cerebral correlates in psychotic disorder
}

Citation for published version (APA):

van der Leeuw, C. (2015). Blood, bones and brains : peripheral biological endophenotypes and their structural cerebral correlates in psychotic disorder. [Doctoral Thesis, Maastricht University]. Maastricht University. https://doi.org/10.26481/dis.20150623cl

Document status and date:

Published: 01/01/2015

DOI:

10.26481/dis.20150623cl

Document Version:

Publisher's PDF, also known as Version of record

Please check the document version of this publication:

- A submitted manuscript is the version of the article upon submission and before peer-review. There can be important differences between the submitted version and the official published version of record.

People interested in the research are advised to contact the author for the final version of the publication, or visit the DOI to the publisher's website.

- The final author version and the galley proof are versions of the publication after peer review.

- The final published version features the final layout of the paper including the volume, issue and page numbers.

Link to publication

\footnotetext{
General rights rights.

- You may freely distribute the URL identifying the publication in the public portal. please follow below link for the End User Agreement:

www.umlib.nl/taverne-license

Take down policy

If you believe that this document breaches copyright please contact us at:

repository@maastrichtuniversity.nl

providing details and we will investigate your claim.
}

Copyright and moral rights for the publications made accessible in the public portal are retained by the authors and/or other copyright owners and it is a condition of accessing publications that users recognise and abide by the legal requirements associated with these

- Users may download and print one copy of any publication from the public portal for the purpose of private study or research.

- You may not further distribute the material or use it for any profit-making activity or commercial gain

If the publication is distributed under the terms of Article $25 \mathrm{fa}$ of the Dutch Copyright Act, indicated by the "Taverne" license above, 


\section{BLOOD, BONES AND BRAINS}

Peripheral biological endophenotypes and

their structural cerebral correlates in psychotic disorder 
ISBN: $\quad$ 978-94-6299-117-0

Print: $\quad$ Ridderprint BV, the Netherlands

Layout: Nikki Vermeulen, Ridderprint BV, the Netherlands

\section{(c) 2015 Christine van der Leeuw}

All rights reserved. No part of this publication may be reproduced or transmitted in any form of by any means, electronic or mechanical, including photocopy, recording, or any information storage or retrieval system, without permission in writing the author. The copyright of the articles that have been accepted for publication or that have already been published, has been transferred to the respective journals. 


\section{BLOOD, BONES AND BRAINS}

Peripheral biological endophenotypes and

their structural cerebral correlates in psychotic disorder

\section{PROEFSCHRIFT}

Ter verkrijging van de graad van doctor aan de Universiteit Maastricht op gezag van de Rector Magnificus Prof. dr. L.L.G. Soete volgens het besluit van het College van Decanen in het openbaar te verdedigen op dinsdag 23 juni 2015 om 14.00 uur

door

Christine van der Leeuw geboren 25 juli 1982 te Geldrop 


\section{Promotor}

Prof. dr. J. van Os

\section{Copromotor}

Dr. M. Marcelis

\section{Beoordelingscommissie}

Prof. dr.T.A.M.J. van Amelsvoort (voorzitter)

Dr. B. Arts

Dr. R. Bruggeman, Universitair Medisch Centrum Groningen

Prof. dr. F.P.M.L. Peeters

Prof. dr. I.E.C. Sommer, Universitair Medisch Centrum Utrecht 


\section{CONTENTS}

Chapter 1 General introduction 7

Chapter 2 Bone mineral density as a marker of cumulative endogenous estrogen

exposure: relationship to background genetic risk of psychotic disorder

Chapter 3 Testing the estrogen hypothesis of schizophrenia: associations between cumulative estrogen exposure and cerebral structural measures

Chapter 4 Bone mineral density as a marker of cumulative estrogen exposure in psychotic disorder: a 3 year follow-up study

Chapter 5 Replicated evidence of absence of association between serum S100B and (risk of) psychotic disorder

Chapter 6 Serum S100B: a proxy marker for grey and white matter status in the absence and presence of (increased risk of) psychotic disorder?

Chapter 7 General discussion

Chapter 8 Summary

Chapter 9 Samenvatting

Chapter 10 Valorization

Dankwoord 


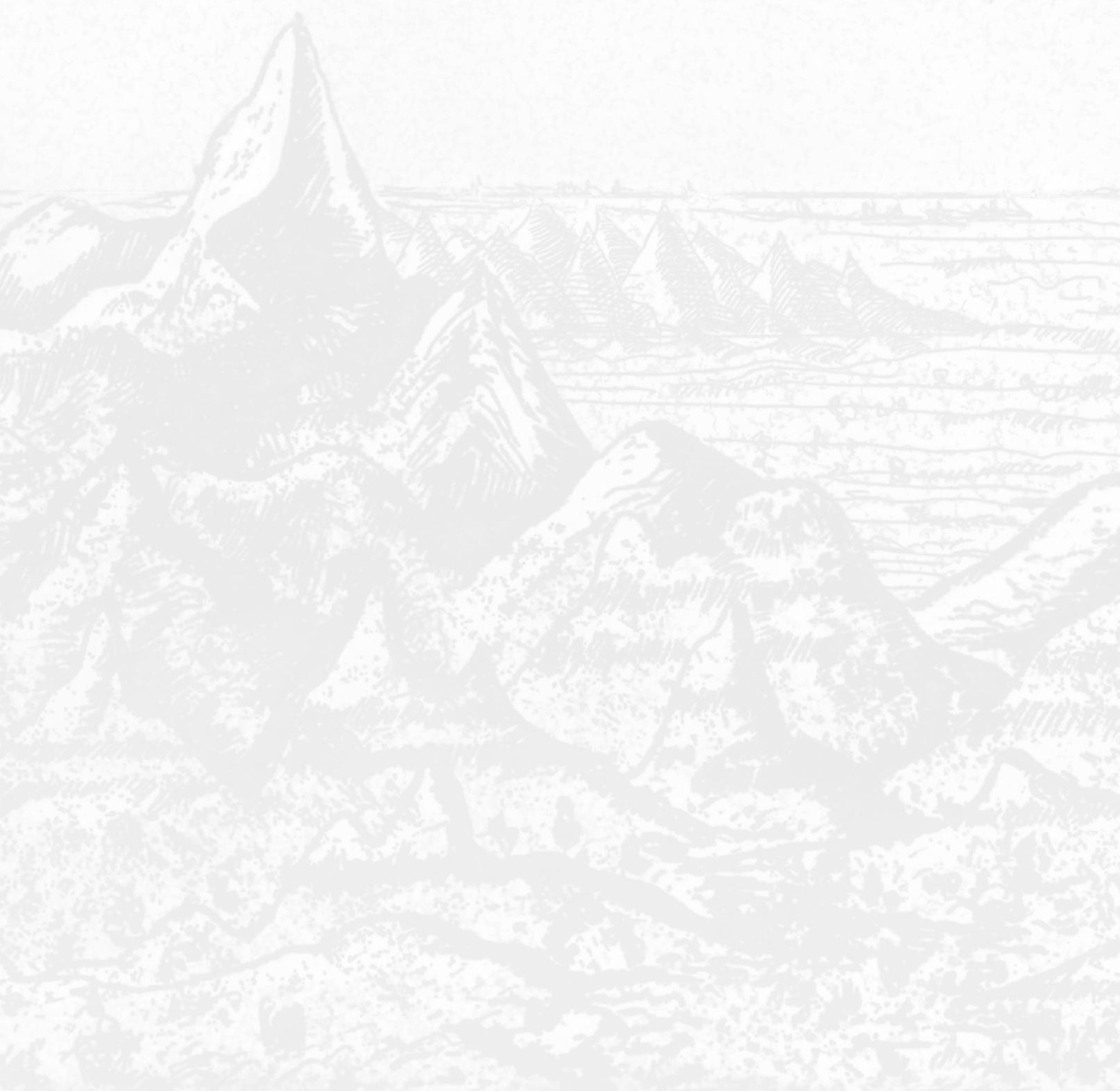




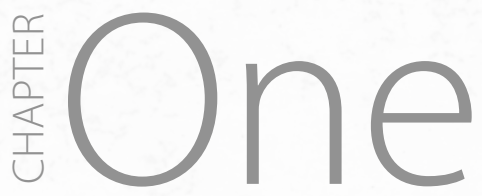

General introduction 



\section{Psychotic disorder}

The term psychotic disorder encompasses multiple syndromes as described in the Diagnostic and Statistical Manual of Mental Disorders (DSM) (APA, 2000), of which schizophrenia is perhaps the foremost diagnosis. Psychotic disorders are characterized by the presence of altered sensory perception (hallucinations) and/or thought content (delusions). Reality testing is inherently compromised. For instance, an individual may perceive a voice that he/she ascribes to being persecuted. Hallucinations and delusions are the so-called positive symptoms of schizophrenia, i.e. phenomena which are present in the context of the disorder. People diagnosed with schizophrenia also experience negative symptoms. Compared to their premorbid condition, certain functions (e.g. initiative and motivation) are diminished. Furthermore, affective and cognitive disturbances are frequently present (van Os and Kapur, 2009).

The classification of psychiatric morbidity according to DSM criteria leads to a categorical diagnosis. You either have it or you don't. However, it is improbable that the DSM's diagnostic categories represent discrete nosological entities with biological substrates (van Os, 2009). The continuum theory states that up to $8 \%$ of all individuals in the general population report psychotic experiences (van Os, 2009; van Os et al., 2009). This implies that subclinical psychosis is a natural phenotype of normality. Whether an individual is diagnosed with a psychotic disorder depends on the duration and intensity of psychotic symptoms and impairment of functioning and daily life.

\section{Epidemiology}

The lifetime prevalence rate of schizophrenia is said to be 1\%, although this figure is based on narrowly defined criteria of the disorder (Castle et al., 1993; van Os and Kapur, 2009). A systematic review of the literature concludes that the overall lifetime prevalence rate is slightly lower at $0.7 \%$ (McGrath et al., 2008). Sex differences in the incidence of schizophrenia are well documented. Overall, men manifest a slightly higher risk of 1.4 (Aleman et al., 2003). In early adulthood, incidence rates are lower in women than in men, and the age at onset of schizophrenia occurs three to four years later in women (Abel et al., 2010; Hafner et al., 1998; Markham, 2012; Riecher-Rossler and Hafner, 1993, 2000). When estrogen levels decline around menopause, women display a second peak in incidence, which is absent in men (Abel et al., 2010; Hafner et al., 1998; Kirkbride et al., 2012; Riecher-Rossler and Hafner, 1993). The epidemiological sex differences in schizophrenia may in part be attributed to the neuroprotective effects of endogenous estrogen in females during their child-bearing years.

\section{Etiology}

Developing a psychotic disorder is the product of heritability and environmental influences, known as gene-environment interaction ( $\mathrm{G} \times \mathrm{E}$ ). Well known environmental risk factors include cannabis use, urbanicity and trauma (van Os et al., 2010). Genetic and environmental risks are thought to converge on a final common pathway to psychotic disorder, on which increased mesolimbic dopamine reactivity 
leads to hallucinations and delusions (van Os et al., 2010), while the role of other neurotranmitters should not be neglected.

Structural magnetic resonance imaging (MRI) studies report progressive changes in brains affected by schizophrenia (Hulshoff Pol and Kahn, 2008; Vita et al., 2012) including an overall decrease in volume, increase in lateral ventricular size and loss of cortical grey matter. Diffusion tensor imaging (DTI) studies in schizophrenia have presented evidence of diminished white matter integrity reflected by decreased fractional anisotropy (FA) (Ellison-Wright and Bullmore, 2009; Kanaan et al., 2005; Kubicki et al., 2007). Consequently, altered structure may cause dysconnectivity which may represent a key pathological mechanism in the disorder (Friston, 1998; Konrad and Winterer, 2008).

It is still largely unknown what causes these changes in the physiology and structure of the brain. Nor are we able to detect the disease processes that lead up to them. The identification of so-called endophenotypes may provide answers.

\section{Endophenotypes}

As complex genetics underlie psychiatric disorders, genotypes do not lead to predictable phenotypes. Endophenotypes, also known as intermediate phenotypes, are traits that lie between genotype and phenotype. They are discrete, genetically determined, variables that present at the subclinical level between genetic risk and the clinical entity of disease (Allen et al., 2009; Bender et al., 2007; Braff et al., 2007). Essentially, endophenotypes can be considered (biological) markers of genetic vulnerability. Theoretically, an endophenotype and clinical disease may share genetic determinants that cause the endophenotype and the clinical disease to coexist independently. This is known as the liability-index or risk indicator model of endophenotypes. Alternatively, the genetic risk for disease is associated with the endophenotype on the causal pathway, which is termed the mediational model of endophenotypes (Kendler and Neale, 2010).

Endophenotypes are "not the signs or symptoms of psychiatry" (Braff et al., 2007). Their identification requires objective quantitative methods (e.g. laboratory-based tests) (Gottesman and Gould, 2003). Endophenotypes may be indicative of underlying pathogenic processes (Allen et al., 2009; Braff et al., 2007). As trait markers they exist independently, irrespective of the manifestation of disease (Gottesman and Gould, 2003).

According to Gottesman and Gold, the following criteria apply to an endophenotype : 1) it is associated with illness in the population, 2) it is heritable, 3) it is state-independent (whether disease is or isn't active), 4) its inheritance is coupled with the disorder within families, and 5) it is found at a higher rate in unaffected relatives than in the general population (Gottesman and Gould, 2003). Establishing endophenotypes offers insight into etiological mechanisms by discerning between primary and secondary disease-effects. This in turn could lead to the development of new treatment perspectives. Furthermore, endophenotypes may provide better grounds for nosology of psychiatric disorders than the currently employed categories of the DSM which do not facilitate the understanding of psychiatry's clinical and biological heterogeneity (Braff et al., 2007; Gottesman and Gould, 2003). 
Endophenotypes may simplify the terminology of complex and heterogeneous syndromes by providing more meaningful and accurate classification based on objective clinical parameters in the individual patient (Allen et al., 2009; Braff et al., 2007).

\section{Candidate endophenotypes in psychotic disorder}

In this thesis, two candidate endophenotypes are examined. In addition, their relation to structural brain measures is tested.

\section{Estrogen exposure}

Deduced from sex differences in the epidemiology and symptomatology of schizophrenia, altered estrogen-mediated neuroprotection may be an endophenotype of psychotic disorder. There are two (complementary) estrogen hypotheses of schizophrenia: 1) the hypoestrogenism or deficiency hypothesis which describes (chronic) gonadal dysfunction in women with schizophrenia, and 2) the protection hypothesis which states that estrogen exerts relative protection against schizophrenia in premenopausal women (Riecher-Rossler, 2002; Riecher-Rossler and Hafner, 1993). In theory, estrogen's neuroprotective effects are also present in men as androgens are aromatized focally, and biologically effective concentrations of estrogen are reached at certain tissue sites including the brain and bone (Boerma et al., 2010).

Bone mineral density (BMD) has been conceptualized as a marker of cumulative estrogen exposure (Clemons and Goss, 2001). Subsequently, BMD may be a peripheral indicator of estrogen-mediated neuroprotection and reduced BMD (reflecting diminished neuroprotection) may be indicative of risk of psychotic disorder. Compatible with this line of reasoning, lumbar BMD was found to be reduced in females presenting with a first episode of psychosis (Maric et al., 2005).

Associations between estrogen exposure and brain structure are well documented in health. Sex steroids have been associated with structural brain development, exerting effects on both white (Asato et al., 2010; Herting et al., 2012; Peper et al., 2011) and grey matter (Neufang et al., 2009; Peper et al., 2009; Peper et al., 2011). Accordingly, gender differences have been reported in healthy brain structure (Goldstein et al., 2001; Kanaan et al., 2012; Kanaan et al., 2014) and normal sexual dimorphism has shown to be disrupted in schizophrenia (Goldstein et al., 2002). Clinical trials provide indirect proof of a neuroprotective impact in schizophrenia. Beneficial effects of estrogen and its receptor modulators as adjunctive therapy for psychosis have been reported in both sexes (Kulkarni et al., 2008; Kulkarni et al., 2011; Kulkarni et al., 2014; Kulkarni et al., 2010), with a quantitative review supporting this effect in women (Begemann et al., 2012).

The investigation of estrogen exposure as an endophenotype will hopefully further the understanding of estrogen-mediated effects in psychotic disorder. It may provide a theoretical and biological basis for the clinical implementation of estrogen augmentation and selective estrogen receptor modulators (SERMs) in addition to antipsychotic treatment. 
A peripheral marker that can provide information about genetic vulnerability for psychosis or that is indicative of altered intracerebral processes or brain status is worth seeking. Serum S100B is a possible candidate. It is a protein that has been described as a potential marker of neurological and psychiatric disease and has been coined the "C-reactive protein (CRP) of the brain" (Sen and Belli, 2007). S100B has dose-dependent effects, at physiologic intracellular levels and nanomolar extracellular concentrations it is neurotrophic (Donato et al., 2013; Donato et al., 2009), at micromolar concentrations it becomes neurotoxic (Sen and Belli, 2007; van Beveren et al., 2006). Whether S100B in psychotic disorder mirrors pathophysiological mechanisms (which elicit exacerbation of disease) or compensatory action is unclear.

Aleksovska and colleagues suggest that S100B is a candidate biomarker of neuroplasticity although there is little literature to illustrate this. One study associated S100B overexpression in transgenic mice with neurogenesis in the hippocampus in stressful conditions (Buschert et al., 2013). In other animal studies, posttraumatic intraventricular infusion of S100B has been shown to benefit hippocampal neurogenesis and cognitive function (Kleindienst et al., 2004; Kleindienst et al., 2005). Human studies are at an earlier stage. Streitburger and colleagues (Streitburger et al., 2012) were the first to test the validity of S100B as a peripheral marker for the human brain. They demonstrated that S100B was negatively correlated with the cerebral white matter integrity marker fractional anisotropy (FA) and positively correlated with radial diffusivity in healthy females, possibly signifying diminished fiber directionality and myelination. These findings have not been replicated thus far. To date, the validity of S100B as a proxy marker for grey and white matter status has not been investigated in patients with a psychotic disorder or other psychiatric morbidity.

Optimistically, the investigation of S100B as an endophenotype of psychotic disorder will prove useful to aid the identification of at-risk individuals and to signal altered intracerebral processes.

\section{Genetic Risk and Outcome of Psychosis (G.R.O.U.P.)}

Data was collected in the context of a naturalistic longitudinal study (Genetic Risk and Outcome of Psychosis, GROUP) in the Netherlands, designed to examine primary (etiological) and secondary factors (consequences of the disease process or its treatment) in the context of psychotic disorder. The GROUP consortium included the academic psychiatric centres of Amsterdam, Groningen, Maastricht and Utrecht, and their affiliations (including several locations in Belgium). Patients with a diagnosis of non-organic non-affective psychosis, their unaffected relatives (parents and siblings) and controls were included. At baseline, 1120 patients, 1057 siblings and 590 controls were included (Korver et al., 2012). The use of non-psychotic relatives in the study design makes it possible to establish the effect of shared familial risk of psychotic disorder, without confounding by disease-related factors. Measurements were conducted at baseline and after 3 years follow-up.

At Maastricht University, an add-on project was performed locally within a subgroup of the GROUP population (i.e., patients with psychotic disorder, siblings of patients with psychotic disorder, and 
healthy controls). Supplementary biomedical data was collected in order to investigate potential endophenotypes. This data included MRI, dual X-ray absorptiometry (DEXA) scans and serum analysis of the protein S100B. This thesis describes studies performed in multiple (Maastricht add-on and multicentre) populations.

\section{Outline and aim of the thesis}

The general aim of this thesis was to examine two potential peripheral biological endophenotypes, i.e. cumulative estrogen exposure (reflected by BMD) and serum levels of the protein S100B, in relation to structural cerebral measures. This was done by comparing both patients with psychotic disorder and their non-psychotic siblings to controls. To validate these proxy markers as indicators of cerebral status, associations between each peripheral marker and indices of brain structure (i.e. grey and white matter represented by cortical thickness (CT) and fractional anisotropy (FA) respectively) were assessed.

\section{Bone mineral density}

In the study described in chapter 2, BMD was used as a marker of lifetime estrogen exposure to examine alterations in estrogen-mediated neuroprotection in relation to varying levels of genetic risk of psychotic disorder. It was hypothesized that BMD would be reduced in both patients and siblings compared to controls, reflecting familial liability and diminished estrogen-mediated neuroprotection. The estrogen hypothesis of schizophrenia was further tested, as outlined in chapter 3. The association between cumulative (lifetime) estrogen exposure and structural brain measures (CT and FA) was explored in female patients. We hypothesized that both CT and FA would be positively associated with $\mathrm{BMD}$, indicative of the neuroprotective effects of estrogen.

After a follow-up period of 3 years, we reassessed BMD in the original sample (chapter 4). We hypothesized that patients would show excessive decline in BMD compared to siblings and controls, reflecting primary disease effects (continuous low estrogen levels). Disproportionate BMD loss in siblings would lend support to hypoestrogenism as an endophenotype of psychotic disorder, uninfluenced by disease-related factors. Potential adverse secondary disease effects were monitored in patients (including AP use), as they may also contribute to reduced BMD.

\section{S100B}

For the first time, S100B was investigated as an endophenotype of psychotic disorder in the study delineated in chapter 5. We inspected whether S100B levels were associated with (familial risk of) psychotic disorder in two large and independent samples. We predicted that S100B would be elevated in both patients and siblings, suggesting that S100B elevation may be considered an endophenotype. To date, the validity of $\mathrm{S100B}$ as a proxy marker of grey and white matter status has not been established in psychotic disorder. The potential association between serum S100B and neuroimaging markers was examined in patients with psychotic disorder, their non-psychotic siblings and controls. Our aim was to assess S100B-associated altered brain structure in the context of increased risk (patients and 
siblings) and disease-effects (patients) of psychotic disorder. In other words, we tested whether a potential association between S100B and brain measures was moderated by psychosis risk (chapter 6). The significance and clinical implications of our findings are critically reviewed in the General discussion (chapter 7). This thesis' studies are summarized in English and Dutch in chapters 8 and 9. A valorization of this work encompassed in this thesis is provided in chapter 10. 


\section{REFERENCES}

Abel, K.M., Drake, R., Goldstein, J.M., 2010. Sex differences in schizophrenia. International review of psychiatry 22, 417-428.

Aleman, A., Kahn, R.S., Selten, J.P., 2003. Sex differences in the risk of schizophrenia: evidence from meta-analysis. Archives of general psychiatry 60, 565-571.

Allen, A.J., Griss, M.E., Folley, B.S., Hawkins, K.A., Pearlson, G.D., 2009. Endophenotypes in schizophrenia: a selective review. Schizophrenia research 109, 24-37.

APA, 2000. Diagnostic and statistical manual of mental disorders, 4 ed. American Psychiatric Association, Washington, DC.

Asato, M.R., Terwilliger, R., Woo, J., Luna, B., 2010. White matter development in adolescence: a DTI study. Cerebral cortex 20, 2122-2131.

Begemann, M.J., Dekker, C.F., van Lunenburg, M., Sommer, I.E., 2012. Estrogen augmentation in schizophrenia: a quantitative review of current evidence. Schizophrenia research 141, 179-184.

Bender, S., Weisbrod, M., Resch, F., 2007. Which perspectives can endophenotypes and biological markers offer in the early recognition of schizophrenia? Journal of neural transmission 114, 1199-1215.

Boerma, M.A., van der Stel, J.C., van Amelsvoort, T., Linszen, D.H., de Haan, L., 2010. [Women, schizophrenia and oestrogen; neurobiological hypotheses and hormonetherapy studies]. Tijdschrift voor psychiatrie 52, 235-244.

Braff, D.L., Freedman, R., Schork, N.J., Gottesman, II, 2007. Deconstructing schizophrenia: an overview of the use of endophenotypes in order to understand a complex disorder. Schizophrenia bulletin 33, 21-32.

Buschert, J., Hohoff, C., Touma, C., Palme, R., Rothermundt, M., Arolt, V., Zhang, W., Ambree, O., 2013. S100B overexpression increases behavioral and neural plasticity in response to the social environment during adolescence. J Psychiatr Res 47, 1791-1799.

Castle, D.J., Wessely, S., Murray, R.M., 1993. Sex and schizophrenia: effects of diagnostic stringency, and associations with and premorbid variables. The British journal of psychiatry : the journal of mental science 162, 658-664.

Clemons, M., Goss, P., 2001. Estrogen and the risk of breast cancer. The New England journal of medicine 344, 276285.

Donato, R., Cannon, B.R., Sorci, G., Riuzzi, F., Hsu, K., Weber, D.J., Geczy, C.L., 2013. Functions of S100 proteins. Curr Mol Med 13, 24-57.

Donato, R., Sorci, G., Riuzzi, F., Arcuri, C., Bianchi, R., Brozzi, F., Tubaro, C., Giambanco, I., 2009. S100B's double life: intracellular regulator and extracellular signal. Biochim Biophys Acta 1793, 1008-1022.

Ellison-Wright, I., Bullmore, E., 2009. Meta-analysis of diffusion tensor imaging studies in schizophrenia. Schizophrenia research 108, 3-10.

Friston, K.J., 1998. The disconnection hypothesis. Schizophrenia research 30, 115-125.

Goldstein, J.M., Seidman, L.J., Horton, N.J., Makris, N., Kennedy, D.N., Caviness, V.S., Jr., Faraone, S.V., Tsuang, M.T., 2001. Normal sexual dimorphism of the adult human brain assessed by in vivo magnetic resonance imaging. Cerebral cortex 11, 490-497.

Goldstein, J.M., Seidman, L.J., O’Brien, L.M., Horton, N.J., Kennedy, D.N., Makris, N., Caviness, V.S., Jr., Faraone, S.V., Tsuang, M.T., 2002. Impact of normal sexual dimorphisms on sex differences in structural brain abnormalities in schizophrenia assessed by magnetic resonance imaging. Archives of general psychiatry 59, 154-164.

Gottesman, II, Gould, T.D., 2003. The endophenotype concept in psychiatry: etymology and strategic intentions. The American journal of psychiatry 160, 636-645.

Hafner, H., an der Heiden, W., Behrens, S., Gattaz, W.F., Hambrecht, M., Loffler, W., Maurer, K., Munk-Jorgensen, P., Nowotny, B., Riecher-Rossler, A., Stein, A., 1998. Causes and consequences of the gender difference in age at onset of schizophrenia. Schizophrenia bulletin 24, 99-113. 
Herting, M.M., Maxwell, E.C., Irvine, C., Nagel, B.J., 2012. The impact of sex, puberty, and hormones on white matter microstructure in adolescents. Cerebral cortex 22, 1979-1992.

Hulshoff Pol, H.E., Kahn, R.S., 2008. What happens after the first episode? A review of progressive brain changes in chronically ill patients with schizophrenia. Schizophrenia bulletin 34, 354-366.

Kanaan, R.A., Allin, M., Picchioni, M., Barker, G.J., Daly, E., Shergill, S.S., Woolley, J., McGuire, P.K., 2012. Gender differences in white matter microstructure. PLoS One 7, e38272.

Kanaan, R.A., Chaddock, C., Allin, M., Picchioni, M.M., Daly, E., Shergill, S.S., McGuire, P.K., 2014. Gender influence on white matter microstructure: a tract-based spatial statistics analysis. PLoS One 9, e91109.

Kanaan, R.A., Kim, J.S., Kaufmann, W.E., Pearlson, G.D., Barker, G.J., McGuire, P.K., 2005. Diffusion tensor imaging in schizophrenia. Biological psychiatry 58, 921-929.

Kendler, K.S., Neale, M.C., 2010. Endophenotype: a conceptual analysis. Mol Psychiatry 15, 789-797.

Kirkbride, J.B., Errazuriz, A., Croudace, T.J., Morgan, C., Jackson, D., Boydell, J., Murray, R.M., Jones, P.B., 2012. Incidence of schizophrenia and other psychoses in England, 1950-2009: a systematic review and meta-analyses. PloS one 7, e31660.

Kleindienst, A., Harvey, H.B., Rice, A.C., Muller, C., Hamm, R.J., Gaab, M.R., Bullock, M.R., 2004. Intraventricular infusion of the neurotrophic protein S100B improves cognitive recovery after fluid percussion injury in the rat. Journal of neurotrauma 21, 541-547.

Kleindienst, A., McGinn, M.J., Harvey, H.B., Colello, R.J., Hamm, R.J., Bullock, M.R., 2005. Enhanced hippocampa neurogenesis by intraventricular $\mathrm{S100B}$ infusion is associated with improved cognitive recovery after traumatic brain injury. Journal of neurotrauma 22, 645-655.

Konrad, A., Winterer, G., 2008. Disturbed structural connectivity in schizophrenia primary factor in pathology or epiphenomenon? Schizophrenia bulletin 34, 72-92.

Korver, N., Quee, P.J., Boos, H.B., Simons, C.J., de Haan, L., 2012. Genetic Risk and Outcome of Psychosis (GROUP), a multi-site longitudinal cohort study focused on gene-environment interaction: objectives, sample characteristics, recruitment and assessment methods. International journal of methods in psychiatric research 21, 205-221.

Kubicki, M., McCarley, R., Westin, C.F., Park, H.J., Maier, S., Kikinis, R., Jolesz, F.A., Shenton, M.E., 2007. A review of diffusion tensor imaging studies in schizophrenia. Journal of psychiatric research 41, 15-30.

Kulkarni, J., de Castella, A., Fitzgerald, P.B., Gurvich, C.T., Bailey, M., Bartholomeusz, C., Burger, H., 2008. Estrogen in severe mental illness: a potential new treatment approach. Archives of general psychiatry 65, 955-960.

Kulkarni, J., de Castella, A., Headey, B., Marston, N., Sinclair, K., Lee, S., Gurvich, C., Fitzgerald, P.B., Burger, H., 2011. Estrogens and men with schizophrenia: is there a case for adjunctive therapy? Schizophrenia research 125, 278-283.

Kulkarni, J., Gavrilidis, E., Wang, W., Worsley, R., Fitzgerald, P.B., Gurvich, C., Van Rheenen, T., Berk, M., Burger, H., 2014. Estradiol for treatment-resistant schizophrenia: a large-scale randomized-controlled trial in women of childbearing age. Molecular psychiatry.

Kulkarni, J., Gurvich, C., Lee, S.J., Gilbert, H., Gavrilidis, E., de Castella, A., Berk, M., Dodd, S., Fitzgerald, P.B., Davis, S.R., 2010. Piloting the effective therapeutic dose of adjunctive selective estrogen receptor modulator treatment in postmenopausal women with schizophrenia. Psychoneuroendocrinology 35, 1142-1147.

Maric, N., Popovic, V., Jasovic-Gasic, M., Pilipovic, N., van Os, J., 2005. Cumulative exposure to estrogen and psychosis: a peak bone mass, case-control study in first-episode psychosis. Schizophrenia research 73, 351-355.

Markham, J.A., 2012. Sex steroids and schizophrenia. Reviews in endocrine \& metabolic disorders 13, $187-207$.

McGrath, J., Saha, S., Chant, D., Welham, J., 2008. Schizophrenia: a concise overview of incidence, prevalence, and mortality. Epidemiologic reviews 30, 67-76.

Neufang, S., Specht, K., Hausmann, M., Gunturkun, O., Herpertz-Dahlmann, B., Fink, G.R., Konrad, K., 2009. Sex differences and the impact of steroid hormones on the developing human brain. Cerebral cortex 19, 464-473. 
Peper, J.S., Brouwer, R.M., Schnack, H.G., van Baal, G.C., van Leeuwen, M., van den Berg, S.M., Delemarre-Van de Waal, H.A., Boomsma, D.I., Kahn, R.S., Hulshoff Pol, H.E., 2009. Sex steroids and brain structure in pubertal boys and girls. Psychoneuroendocrinology 34, 332-342.

Peper, J.S., Hulshoff Pol, H.E., Crone, E.A., van Honk, J., 2011. Sex steroids and brain structure in pubertal boys and girls: a mini-review of neuroimaging studies. Neuroscience 191, 28-37.

Riecher-Rossler, A., 2002. Oestrogen effects in schizophrenia and their potential therapeutic implications--review. Archives of women's mental health 5, 111-118.

Riecher-Rossler, A., Hafner, H., 1993. Schizophrenia and oestrogens--is there an association? European archives of psychiatry and clinical neuroscience 242, 323-328.

Riecher-Rossler, A., Hafner, H., 2000. Gender aspects in schizophrenia: bridging the border between social and biological psychiatry. Acta psychiatrica Scandinavica. Supplementum, 58-62.

Sen, J., Belli, A., 2007. S100B in neuropathologic states: the CRP of the brain? J Neurosci Res 85, 1373-1380.

Streitburger, D.P., Arelin, K., Kratzsch, J., Thiery, J., Steiner, J., Villringer, A., Mueller, K., Schroeter, M.L., 2012. Validating Serum S100B and Neuron-Specific Enolase as Biomarkers for the Human Brain - A Combined Serum, Gene Expression and MRI Study. PLoS One 7, e43284.

van Beveren, N.J., van der Spelt, J.J., de Haan, L., Fekkes, D., 2006. Schizophrenia-associated neural growth factors in peripheral blood. A review. Eur Neuropsychopharmacol 16, 469-480.

van Os, J., 2009. 'Salience syndrome' replaces 'schizophrenia' in DSM-V and ICD-11: psychiatry's evidence-based entry into the 21st century? Acta psychiatrica Scandinavica 120, 363-372.

van Os, J., Kapur, S., 2009. Schizophrenia. Lancet 374, 635-645.

van Os, J., Kenis, G., Rutten, B.P., 2010. The environment and schizophrenia. Nature 468, $203-212$.

van Os, J., Linscott, R.J., Myin-Germeys, I., Delespaul, P., Krabbendam, L., 2009. A systematic review and meta-analysis of the psychosis continuum: evidence for a psychosis proneness-persistence-impairment model of psychotic disorder. Psychological medicine 39, 179-195.

Vita, A., De Peri, L., Deste, G., Sacchetti, E., 2012. Progressive loss of cortical gray matter in schizophrenia: a metaanalysis and meta-regression of longitudinal MRI studies. Translational psychiatry 2, e190. 
a Department of Psychiatry \& Psychology, School for Mental Health and Neuroscience, EURON, Maastricht University Medical Centre, PO Box 616, 6200 MD Maastricht, the Netherlands

b Department of Nuclear Medicine, Maastricht University Medical Centre, Maastricht, the Netherlands

c Visiting Professor of Psychiatric Epidemiology, King's College London, King's Health Partners, Department of Psychosis Studies, Institute of Psychiatry, London, UK 


\section{辜TWO}

Bone mineral density as a marker of cumulative endogenous estrogen exposure: relationship to background genetic risk of psychotic disorder.

C. van der Leeuwa ${ }^{M D}$

P. Habets ${ }^{a}$ PhD

P. Domen ${ }^{\mathrm{a}} \mathrm{MD}$

M. van Kroonenburgh ${ }^{b}$ MD PhD

J. van $\mathrm{Os}^{\mathrm{a}, \mathrm{c}} \mathrm{MD} \mathrm{PhD}$

M. Marcelis ${ }^{\mathrm{a}}$ MD PhD; for G.R.O.U.P. 


\section{ABSTRACT}

Background Alterations in bone mineral density (BMD) in patients with psychotic disorder may reflect the effect of treatment (disease effect observed in patients but not their siblings) or, as an intermediate marker of cumulative endogenous estrogen exposure, alterations in the neuroprotective effect of estrogen in the brain (vulnerability effect observed in patients and siblings).

Methods Dual X-ray absorptiometry (DEXA) scans were acquired in 62 patients with a psychotic disorder, 67 non-psychotic siblings of patients with a psychotic disorder, and 48 controls. BMD (g/ $\left.\mathrm{cm}^{2}\right)$, Z-scores and T-scores were measured in the lumbar spine and proximal femur. Associations between group and BMD were investigated with multilevel random regression analyses. Group $\times$ sex interactions and effects of antipsychotic medication (AP) on BMD were examined.

Results Group was not associated with BMD outcome measures, although patients had consistently lower BMD measures compared to both siblings and controls. There were no significant group $\times$ sex interactions, but stratified analyses showed that BMD measures in female patients were significantly lower in comparison to female controls and siblings (e.g. total femoral $B M D, P$ vs. $C$ : $B=-0.100, p=0.010$; $P$ vs. $S: B=-0.104, p=0.008$ ). After excluding female patients who used prolactin-raising $A P$, the effect was attenuated (e.g. total femoral $B M D, P$ vs. $C: B=-0.073, p=0.072 ; P$ vs. $S: B=-0.085, p=0.051$ ). In men, there were no significant $B M D$ differences between patients and controls.

Conclusions Familial risk of psychotic disorder was not associated with BMD. Instead, decreased $\mathrm{BMD}$ in the femur may reflect treatment effects or non-familial risk associated with low cumulative endogenous estrogen levels in women. 


\section{INTRODUCTION}

In the past decade, an increased awareness of compromised skeletal status in patients with psychotic disorder has developed. This is based on findings of reduced bone mineral density (BMD) in this population, possibly resulting from a high prevalence of risk factors for osteoporosis (Haddad and Wieck, 2004; Halbreich, 2007). Some risk factors for osteoporosis may be inherent to positive and negative symptomatology such as polydipsia (Delva et al., 1989), diminished dietary intake, decreased physical activity and reduced sunlight exposure. In addition, comorbid substance abuse may further influence vulnerability for osteoporosis, as alcohol and cannabis have been shown to affect bone metabolism (Bab et al., 2009; Maurel et al., 2012). Hyperprolactinemia-induced hypogonadism, secondary to treatment with potent dopamine-2 receptor antagonists, has also been proposed as a factor contributing to loss of bone mass in schizophrenia (Abraham et al., 2003a; Bilici et al., 2002; Kishimoto et al., 2008; Meaney and O'Keane, 2007; Meaney et al., 2004). Notably, not all studies have found evidence for BMD reduction in patients (Howes et al., 2005), or for an effect of prolactin-raising antipsychotic drugs (AP) on BMD (Lee et al., 2010; Renn et al., 2010).

While there is evidence for decreased BMD specifically in male patients with schizophrenia (Hummer et al., 2005; Kishimoto et al., 2008; Lehman and Meyer, 2005; Meyer and Lehman, 2006), there are also studies suggesting that female patients are particularly vulnerable to compromised skeletal status (Partti et al., 2010), with some evidence for higher bone turnover, but normal bone mineral density (Abraham et al., 2003b; Bergemann et al., 2008).

The above mentioned studies (all cross-sectional, with the exception of two (Abraham et al., 2003b; Meaney and O'Keane, 2007)), differ with respect to cohort characteristics (e.g. sample size, stage of illness, male and/or female participants, women in pre- or postmenopausal phase) and correction for confounding factors, which may have contributed to an inconsistent pattern of results. Nevertheless, most studies are suggestive of reduced BMD in schizophrenia.

Loss of BMD may be a consequence of psychotic disorder or its treatment, but an alternative hypothesis is that a reduction in BMD, as a marker of cumulative endogenous estrogen exposure (Clemons and Goss, 2001), is an indicator of risk for and development of psychotic disorder (due to diminished neuroprotection of estrogen in the brain) (Maric et al., 2005). A primary low endogenous estrogen level may be associated with genetic risk of the disorder (intermediate phenotype) or, alternatively, represent a non-familial marker of risk.

The aim of the present study was to examine BMD in subjects at high genetic risk of psychotic disorder (patients with psychotic disorder) and subjects at higher than average genetic risk (unaffected siblings of patients with psychotic disorder) in comparison with individuals at average genetic risk of psychotic disorder (healthy controls). We hypothesized that BMD, as a marker of cumulative endogenous estrogen exposure, would be reduced in both patients and siblings, reflecting shared familial liability. The objective was not to identify a large, clinically relevant decline in BMD (e.g. to be able to pinpoint and reduce fracture risk), but to discern a difference, even small, that may be related to the (familial) etiology of schizophrenia and other psychotic disorders. 


\section{METHODS AND MATERIALS}

\section{Participants}

Data was collected in the context of an ongoing longitudinal study in Maastricht, the Netherlands (Habets et al., 2011; Korver et al., 2012). In selected representative geographic areas in the Netherlands and Belgium, patients presenting consecutively at mental health services either as outpatients or inpatients were recruited for the study. Siblings were sampled through participating patients. Control subjects were recruited from the same population as the cases using random mailings in nearby municipalities and through advertisement in newspapers. Patients between the ages of 16 and 50 years with a diagnosis of non-organic, non-affective psychosis were included. Sufficient command of the Dutch language was an additional criterion for inclusion. Diagnosis was based on DSM-IV criteria (APA, 2000), assessed with the Comprehensive Assessment of Symptoms and History (CASH) interview (Andreasen et al., 1992). The CASH was also used to confirm the absence of a diagnosis of non-affective psychosis in the siblings, and absence of a diagnosis of any psychotic disorder in the control subjects. For the control subjects, the occurrence of any psychotic disorder in either the subject or any first-degree family member, assessed using the Family Interview for Genetic Studies, constituted an exclusion criterion.

The sample consisted of 62 patients with a psychotic disorder (of whom 49 patients with a diagnosis of schizophrenia, 4 patients with a diagnosis of schizoaffective disorder, 8 patients with a diagnosis of psychotic disorder not otherwise specified and 1 patient with a diagnosis of brief psychotic disorder), 67 non-psychotic siblings and 48 controls. The sample included 36 families, of which 24 families contributed one patient and one sibling and two families contributed one patient and 2 siblings. One family contributed two patients and one sibling; in one family one patient and 3 siblings participated. Two families contributed two siblings but no patients. In the control group, six families contributed two siblings. Thirty-three independent patients, 31 independent siblings, and 36 independent controls participated.

Nine controls and sixteen siblings had a history of major depressive disorder (MDD). In addition, one sibling was diagnosed with dysthymic disorder and one with mood disorder due to a general medical condition.

Prior to DEXA acquisition, participants were screened for the following exclusion criteria: 1) metabolic or endocrinologic disease, 2) dietary deficiency or eating disorder, 3) medication use: corticosteroids, thyroxin, anti-epileptics, heparin, lithium, cytostatic agents, 4) (semi-) professional athletes, 5) polydipsia (>3 liters / day), 6) pregnancy, and 7) hormonal (infertility) treatment. 


\section{Measures}

\section{Substance use}

Substance use was assessed using the Composite International Diagnostic Interview (CIDI) (WHO, 1990). Cannabis use was assessed as reported lifetime frequency of use. Other drug use, such as stimulants, sedatives, opiates, cocaine, PCP, psychedelics, inhalants or other (e.g. ecstasy, poppers) was assessed in the same way. Alcohol use was defined as the average number of weekly instances of consumption during the previous 12 months. Tobacco use was defined as the number of cigarettes, cigars or pipes per day, or number of daily occasions of use of chewing tobacco or snuff.

\section{Physical activity and sunlight exposure}

Physical activity and sunlight exposure were expressed in total minutes per week.

To quantify physical activity, the total amount of time spent on commuting by foot or bicycle, physical activity at work or school, household chores, active hobbies and sports was summed. Sunlight exposure was calculated by multiplying the number of days per week a person went outside by the average number of minutes spent outside on those days.

APuse

Current AP use was classified by AP type:"prolactin-raising", i.e. typical APs, risperidone and amisulpride; or "prolactin-sparing", i.e. atypical APs with the exception of risperidone and amisulpride.

\section{Use of contraceptive drugs (cumulative exogenous estrogen exposure)}

Cumulative exogenous estrogen exposure in women was determined by multiplying the daily dose with the total days of use, expressed in micrograms.

\section{Age at menarche and dysmenorrhea}

Age at menarche and the occurrence of menstrual irregularity were assessed in women. Dysmenorrhea was defined as altered duration and/or frequency of menses or the absence of two or more menses during the previous three months. Amenorrhea was defined as the absence of menses for at least three months.

\section{Familial osteoporosis}

The occurrence of familial osteoporosis was documented. 


\section{DEXA acquisition and processing}

DEXA scans were acquired at the Maastricht University Medical Centre with a Hologic Discovery A (Tromp Medical, Castricum, the Netherlands) (NHANES and Ethnic Reference Data). DEXA scans of two anatomical areas were performed: the lumbar spine, vertebrae $L 2$ through $L 4$; and the proximal left femur, specifically the collum, trochanter major, intertrochanteric area and Ward's triangle. BMD was expressed in grams per square centimeter $\left(\mathrm{g} / \mathrm{cm}^{2}\right)$, Z-scores and T-scores. The Z-score compares an individual's BMD with the mean BMD of a comparable population (with respect to gender, age and ethnicity). The T-score compares an individual's BMD to peak bone mass (PBM). Peak bone mass is the highest BMD an individual is expected to acquire during life. The T-score is used to diagnose osteopenia and osteoporosis. The World Health Organization employs the following criteria: T-scores in osteopenia lie between -1.0 and -2.5; T-scores in osteoporosis are equal or less than -2.5.

This study was approved by the standing ethics committee. All subjects provided written informed consent in accordance with the committee's guidelines.

\section{Statistical analyses}

Group differences in total BMD, Z-scores andT-scores were analyzed separately for the lumbar spine and proximal femur. Multilevel random regression models were fitted (Goldstein, 1987) given hierarchical clustering occasioned by the fact that participants were clustered in families, compromising statistical independence of the observations. This was done using the XTREG command in STATA (STATA corp, version 11). BMD measures were the dependent variables in the analyses and group (entered both as linear and dummy variables (controls=0, siblings $=1$ and patients $=2$ )) was the independent variable. Analyses were adjusted for a priori hypothesized confounders: cumulative exogenous estrogen exposure, gender, age, BMI, physical activity, sunlight exposure, tobacco and alcohol use, and lifetime exposure to cannabis.

To test whether BMD measures were not only conditional on group but also on sex, group $\times$ sex interactions were examined. In addition, a priori stratified analyses for males and females were conducted separately, given strong physiological sex differences in bone metabolism, possibly violating assumptions of underlying homogeneity in mechanisms and associations. Group $\times$ sex interaction terms were evaluated by Wald test (Clayton and Hills, 1993). In the analyses stratified by sex, age at menarche and menstrual irregularity were additional covariates in the model of women. The study population included nine control subjects and eighteen siblings with a history of affective disorder. Conceptually, these individuals may be at risk of decreased BMD due to factors also associated with psychotic disorder, such as inactivity and diminished sunlight exposure. In addition, selective serotonin reuptake inhibitors (SSRIs) potentially reduce BMD (Halbreich, 2007). Therefore, planned sensitivity analyses were conducted excluding these individuals.

Main effects of current type of AP exposure on BMD in patients were examined by comparing prolactinsparing and prolactin-raising APs to no $\mathrm{AP}$ (dummy variable: no $\mathrm{AP}=0$, prolactin-raising $=1$, prolactin- 
sparing=2) using multiple regression procedures; with sex, age and BMI as potential confounders. Planned sensitivity analyses were performed excluding patients who used a prolactin-raising AP. To control for a type I error, all significant p-values were subjected to correction for multiple testing using the Simes method (Rodland, 2006; Simes, 1986). The Simes method avoids overcorrection associated with the Bonferroni correction in case the statistical tests are not independent, as was the case in the present study (BMD measures are correlated with each other). P-values were ranked and Simes $p$-values were calculated as $j^{*} a l p h a / k$, where $k$ is the number of $p$-values in the ranking and $j$ is the ranking of that $p$-value. The adjusted $p$-value is that of the lowest ranking for which $P<P$ adjusted (hereafter: $P_{\text {simes }}$ ).

\section{RESULTS}

\section{Descriptive analyses}

There were more female participants in the control group and more males in the patient group. Controls were slightly older than patients and siblings. Patients used more tobacco, as well as more cannabis and other drugs, compared to controls and siblings. Siblings used more cannabis than controls. Among women, patients experienced more menstrual irregularities than siblings and controls (Table 1).

Table 1. Demographics

\begin{tabular}{llll}
\hline & Controls $\mathbf{n}=\mathbf{4 8}$ & Siblings $\mathbf{n}=\mathbf{6 7}$ & Patients $\mathbf{n}=\mathbf{6 2}$ \\
\hline Sex (n), M / F & $13 / 35$ & $34 / 33$ & $46 / 16$ \\
Age at scan & $31.17 \pm 11.43$ & $28.18 \pm 7.86$ & $27.42 \pm 6.39$ \\
Body Mass Index (BMI) (kg per m ${ }^{2}$ ) & $24.31 \pm 4.34$ & $23.60 \pm 4.12$ & $24.19 \pm 4.04$ \\
Age at menarche (years) & $13.14 \pm 1.56$ & $12.84 \pm 1.30$ & $13.0 \pm 0.93$ \\
Dysmenorrhea (n) & 6 & 3 & 8 \\
Lifetime exogenous estrogen exposure ( $\mu \mathrm{g})$ & $41140 \pm 44105$ & $41456 \pm 42494$ & $25739 \pm 43081$ \\
Smoking (cigarettes per day) & $1.98 \pm 5.42$ & $3.80 \pm 7.39$ & $12.24 \pm 10.51$ \\
Alcohol consumption (units per week) & $5.50 \pm 7.62$ & $9.39 \pm 18.11$ & $6.57 \pm 14.88$ \\
Cannabis use (number of times lifetime) & $8.87 \pm 23.32$ & $23.94 \pm 40.09$ & $51.92 \pm 47.51$ \\
Other drug use (number of times lifetime) & $0.28 \pm 1.17$ & $9.94 \pm 37.92$ & $54.70 \pm 96.41$ \\
Activity (minutes per week) & $1975 \pm 1112$ & $1800 \pm 1377$ & $1555 \pm 1283$ \\
Sunlight exposure (minutes per week) & $1270 \pm 930$ & $1252 \pm 1127$ & $942 \pm 596$ \\
Familial cases of osteoporosis (n) & 3 & 6 & 7 \\
Current AP use (n, patients) & - & - & 56 \\
PRL-raising / PRL-sparing (n) & & & $28 / 28$ \\
\hline
\end{tabular}

Mean \pm standard deviation (SD) reported.

AP: antipsychotic medication; PRL: prolactin. 
Out of 62 patients, 56 currently used AP medication, of whom 28 were prescribed prolactin-raising agents (3 patients used haloperidol, 1 zuclopenthixol, 17 risperidone and 7 amisulpride; of the latter 1 patient used a combination of amisulpride and aripiprazole, 1 used a combination of amisulpride and haloperidol and 1 patient used a combination of amisulpride and risperidone). Sixteen patients used psychotropic medication other than an AP: twelve used antidepressants (of which 11 a SSRI or serotonin and noradrenalin reuptake inhibitor (SNRI), and 1 trazodone), three used benzodiazepines, two used anticholinergic agents, and there was one occasion of stimulant (methylphenidate) use. Two control subjects used an SSRI. None of the siblings used psychotropic medication.

\section{Mean BMD measures and prevalence of osteopenia / osteoporosis}

The raw data showed that mean BMD measures in patients were lower than those in siblings and controls. Siblings had consistently lower mean BMD measures than controls in the lumbar spine, but not the femur. Compared to controls, the proportion of osteopenia and osteoporosis cases in the group of patients and siblings was larger, albeit not statistically significant (Table 2).

Table 2. Mean BMD measures and point prevalence of osteopenia and osteoporosis

\begin{tabular}{|c|c|c|c|c|c|c|c|c|c|}
\hline & \multicolumn{3}{|c|}{ Controls } & \multicolumn{3}{|c|}{ Siblings } & \multicolumn{3}{|c|}{ Patients } \\
\hline & Total & Male & Female & Total & Male & Female & Total & Male & Female \\
\hline \multicolumn{10}{|l|}{ Lumbar spine } \\
\hline \multirow[t]{2}{*}{$\operatorname{BMD}\left(\mathrm{g} / \mathrm{cm}^{2}\right)$} & 1.076 & 1.051 & 1.085 & 1.072 & 1.074 & 1.069 & 1.046 & 1.051 & 1.033 \\
\hline & $(0.14)$ & $(0.15)$ & $(0.13)$ & $(0.12)$ & $(0.13)$ & $(0.11)$ & $(0.13)$ & $(0.14)$ & $(0.10)$ \\
\hline \multirow[t]{2}{*}{ Z-score } & 0.156 & -0.400 & 0.363 & -0.133 & -0.291 & 0.034 & -0.474 & -0.561 & -0.225 \\
\hline & $(1.29)$ & $(1.36)$ & $(1.22)$ & $(1.12)$ & (1.19) & $(1.04)$ & $(1.23)$ & $(1.31)$ & $(0.99)$ \\
\hline \multirow[t]{2}{*}{ T-score } & -0.081 & -0.569 & 0.100 & -0.202 & -0.341 & -0.053 & -0.527 & -0.583 & -0.369 \\
\hline & $(1.25)$ & $(1.32)$ & $(1.19)$ & $(1.12)$ & $(1.19)$ & $(1.03)$ & $(1.22)$ & $(1.30)$ & $(0.95)$ \\
\hline Osteopenia (n) & 9 & 4 & 5 & 15 & 9 & 6 & $19^{a}$ & 15 & 4 \\
\hline Osteoporosis (n) & 1 & 1 & 0 & 1 & 1 & 0 & 2 & 2 & 0 \\
\hline \multicolumn{10}{|l|}{ Femur } \\
\hline \multirow[t]{2}{*}{$\mathrm{BMD}\left(\mathrm{g} / \mathrm{cm}^{2}\right)$} & 0.988 & 1.042 & 0.967 & 1.017 & 1.064 & 0.967 & 0.987 & 1.004 & 0.937 \\
\hline & $(0.12)$ & $(0.10)$ & $(0.12)$ & $(0.15)$ & $(0.15)$ & $(0.12)$ & $(0.15)$ & $(0.16)$ & $(0.11)$ \\
\hline \multirow[t]{2}{*}{ Z-score } & 0.238 & 0.115 & 0.283 & 0.180 & 0.235 & 0.122 & -0.144 & -0.170 & -0.069 \\
\hline & $(0.90)$ & $(0.70)$ & $(0.97)$ & $(1.04)$ & $(1.11)$ & $(0.98)$ & $(1.04)$ & $(1.06)$ & $(1.03)$ \\
\hline \multirow[t]{2}{*}{ T-score } & 0.081 & -0.092 & 0.146 & 0.094 & 0.126 & 0.059 & -0.206 & -0.235 & -0.125 \\
\hline & $(0.93)$ & $(0.75)$ & $(0.99)$ & $(1.05)$ & $(1.11)$ & $(1.00)$ & $(1.05)$ & $(1.07)$ & $(1.02)$ \\
\hline Osteopenia (n) & 6 & 2 & 4 & 9 & 4 & 5 & $15^{b}$ & 11 & 4 \\
\hline Osteoporosis (n) & 0 & 0 & 0 & 0 & 0 & 0 & 0 & 0 & 0 \\
\hline
\end{tabular}

Means \pm SDs reported.

ane-sided Fisher's exact test (patients vs. controls): 0.115.

bOne-sided Fisher's exact test (patients vs. controls): 0.095 . 


\section{Associations between group and BMD measures of the lumbar spine and femur}

There was no statistically significant association between group (linear trend) and BMD, although effect sizes were negative for all six BMD measures. In the patient-control comparison, the direction of the effect size was consistently negative for all six BMD measures of the lumbar spine and femur, but did not reach significance. Similarly, there were no significant findings in the sibling-control comparison. Comparing patients to siblings, there was a negative association for all BMD measures. The reduction in total $\mathrm{BMD}$ and $\mathrm{Z}$-score of the femur was significant $(\mathrm{B}=-0.061,95 \% \mathrm{Cl}:-0.117$ to $-0.005, p=0.033$; and $B=-0.414,95 \% C l:-0.827$ to $-0.001, p=0.050$ respectively), though not after Simes correction. The reduction of the femoral T-score did not reach significance $(B=-0.384,95 \% \mathrm{Cl}:-0.797$ to $0.030, p=0.069$ ) (Table 3).

Table 3. Associations between increased genetic risk of psychotic disorder (group) and BMD

\begin{tabular}{|c|c|c|c|c|c|c|c|c|}
\hline & \multicolumn{2}{|c|}{ Linear trend } & \multicolumn{2}{|l|}{ S vs. C } & \multicolumn{2}{|l|}{ P vs. C } & \multicolumn{2}{|l|}{ P vs. S } \\
\hline & B & $p$-value & B & $p$-value & B & $p$-value & B & $p$-value \\
\hline \multicolumn{9}{|l|}{ Lumbar spine } \\
\hline Total BMD (g/cm²) & -0.006 & 0.736 & 0.004 & 0.872 & -0.013 & 0.693 & -0.017 & 0.552 \\
\hline Z-score & -0.122 & 0.451 & -0.050 & 0.842 & -0.253 & 0.414 & -0.203 & 0.456 \\
\hline T-score & -0.068 & 0.668 & 0.049 & 0.841 & -0.151 & 0.617 & -0.201 & 0.452 \\
\hline \multicolumn{9}{|l|}{ Femur } \\
\hline Total BMD $\left(\mathrm{g} / \mathrm{cm}^{2}\right)$ & -0.027 & 0.111 & 0.002 & 0.927 & -0.058 & 0.070 & -0.061 & $0.033^{\mathrm{a}}$ \\
\hline Z-score & -0.207 & 0.100 & -0.025 & 0.897 & -0.439 & 0.067 & -0.414 & $0.050^{\mathrm{a}}$ \\
\hline T-score & -0.178 & 0.159 & 0.004 & 0.985 & -0.380 & 0.114 & -0.384 & 0.069 \\
\hline
\end{tabular}

The Bs represent the regression coefficients of the multilevel regression analyses.

S vs. C: siblings vs. controls; P vs. C: patients vs. controls; P vs. S: patients vs. siblings.

aStatistical significance not upheld after Simes correction.

\section{Group $\times$ sex interaction and stratified analyses by sex}

There were no significant group $\times$ sex interactions for the lumbar spine or proximal femur. In women, all BMD measures of the femur were significantly reduced in patients compared to controls and siblings, with no significant differences between siblings and controls. These findings held after Simes correction $\left(P_{\text {simes }}: p<0.017\right)$.

In men, no significant associations were found between BMD and group. The direction of the effect was generally positive in the lumbar spine, but consistently negative in the femur in male patients and siblings compared to controls (Table 4). 


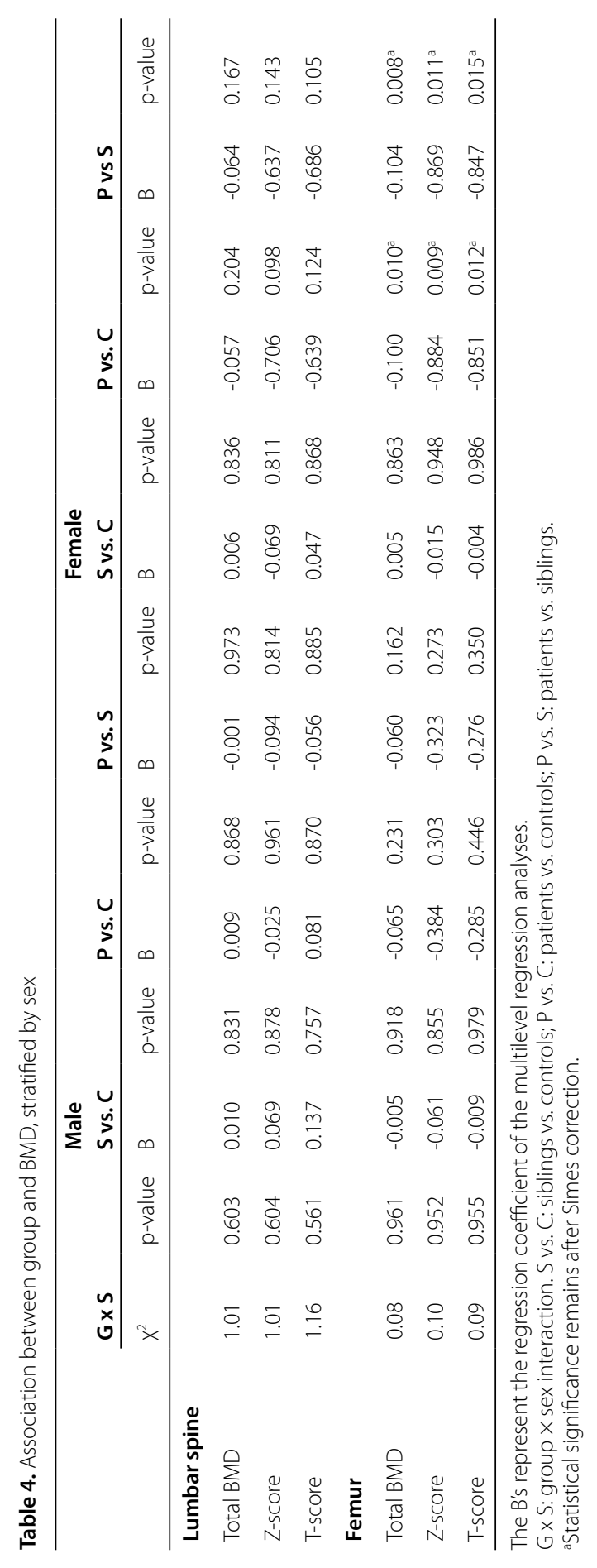




\section{Associations between antipsychotic medication and BMD}

In the total patient group, no significant associations between the type of current AP and BMD were found, when compared to AP-free patients $(n=5)$. In women, but not in men, the current use of a prolactin-raising AP $(n=6)$ appeared to be negatively associated with BMD measures of the lumbar spine, when compared to AP-free female patients $(n=3)$ (total $B M D: B=-0.113,95 \% C l:-0.220$ to -0.006 , $p=0.041$; Z-score: $B=-1.109,95 \% C l:-2.243$ to $0.025, p=0.054$; T-score: $B=-1.199,95 \%$ Cl: -2.236 to $-0.162, p=0.027)$. Results were not upheld after Simes correction. No such association was found for the femur.

\section{Exclusion of affective disorder and prolactin-raising AP}

Repeating the analyses excluding siblings and controls with a history of affective disorder did not affect the findings in the total group $(n=150)$, or in men $(n=87)$, and thus remained negative. In women $(n=63)$, the significance level of the negative association between group (patients) and femoral BMD was enhanced (see effect sizes and p-values in Figure 1). These findings held after Simes correction $\left(P_{\text {Simes }}: p<0.017\right)$.

Figure 1. Modelled effects of genetic risk of psychotic disorder (group) on femoral BMD in women

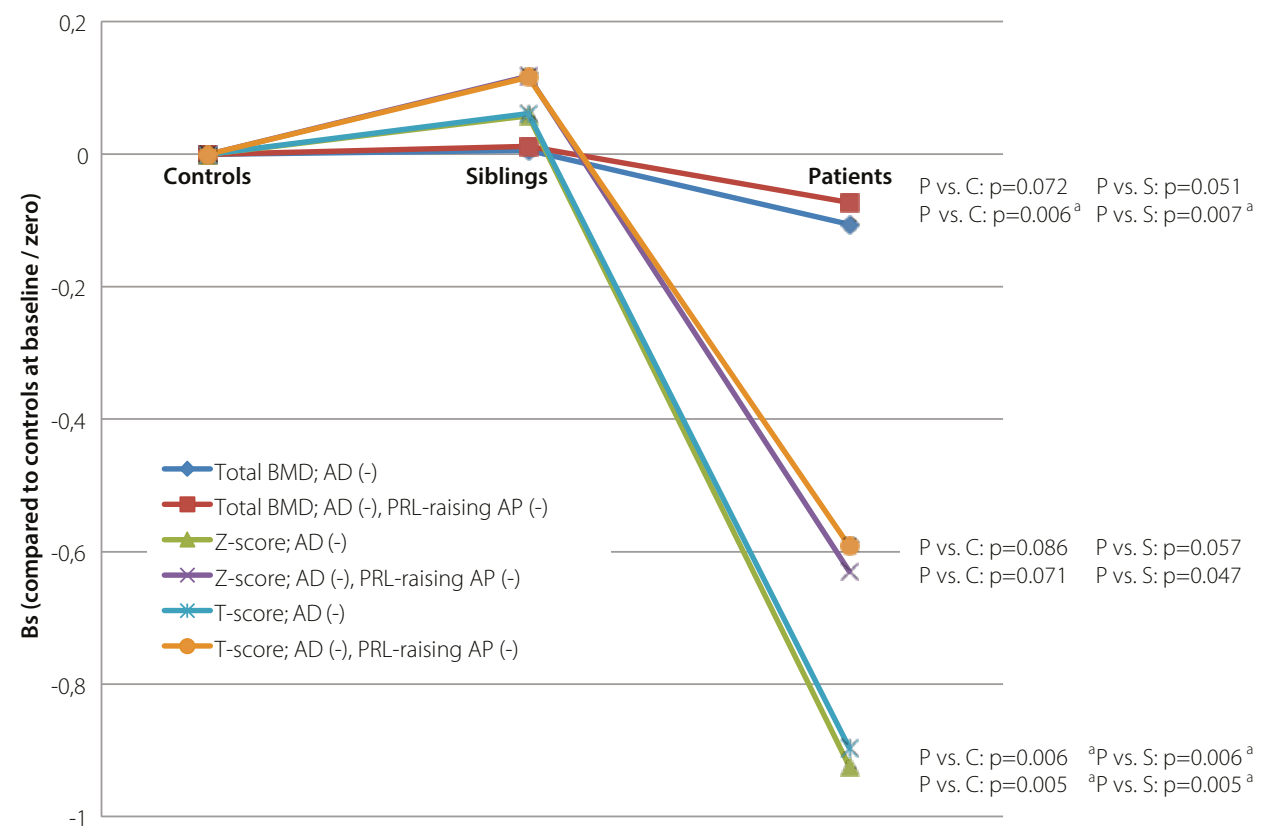

Bs are compared to controls represented as zero.

AD (-): exclusion of affective disorder among controls and siblings; PRL-raising (-): exclusion of prolactin-raising APs. aStatistical significance remains after Simes correction. 
In addition, a negative association in the lumbar spine became apparent in the patient versus control comparison, although this was not upheld after Simes correction (patients versus controls, total BMD: $B=-0.079,95 \% C l:-0.171$ to $0.013, p=0.093 ; Z$-score: $B=-0.888,95 \% C l-1.756$ to $-0.021, p=0.045$; T-score: $\mathrm{B}=-0.839,95 \% \mathrm{Cl}-1.672$ to $-0.005, \mathrm{p}=0.049$ ). Female siblings were not significantly different from female controls or patients for all BMD measures.

Analyses were once again repeated after excluding the six female patients who used a prolactinraising AP. Ten female patients remained in the sample. Femoral BMD remained decreased in patients compared to siblings at a trend-level of significance (total BMD: $B=-0.085,95 \% \mathrm{Cl}-0.171$ to 0.000 , $p=0.051$; Z-score: $B=-0.748,95 \% C l-1.484$ to $-0.011, p=0.047$; T-score: $B=-0.708,95 \% C l-1.437$ to 0.021 , $\mathrm{p}=0.057$ ), although effect sizes decreased with $23 \%, 24 \%$ and $26 \%$ respectively. The reduction in BMD in patients compared to controls was no longer significant (total BMD: $B=-0.073,95 \% \mathrm{Cl}:-0.152$ to 0.006, $p=0.072 ; Z$-score: $B=-0.630,95 \% \mathrm{Cl}-1.313$ to $0.054, p=0.071$; T-score: $B=-0.592,95 \% \mathrm{Cl}-1.268$ to $0.084, p=0.086$ ), as effect sizes decreased with respectively $31 \%, 32 \%$ and $34 \%$ (Figure 1).

\section{DISCUSSION}

This study examined BMD as a potential biological marker of cumulative endogenous estrogen exposure in patients with a psychotic disorder, non-psychotic siblings of patients with a psychotic disorder and controls. In the entire sample, group was not conclusively associated with BMD outcome measures, however BMD was directionally lower in patients compared to controls and siblings. Significantly decreased BMD was found specifically in the femur of female patients. After exclusion of patients with prolactin-raising AP, the reduction in femoral BMD was attenuated.

\section{Findings}

Total group (men and women)

Contrary to the initial hypothesis, individuals at higher than average and highest genetic risk for psychotic disorder did not have significantly decreased BMD measures compared to controls. Although patients showed consistently lower BMD measures than controls and siblings, the associations were statistically inconclusive or reached trend-level significance. While most previous studies are indicative of reduced BMD in schizophrenia, some (Howes et al., 2005) did not find a significant reduction in BMD in patients with schizophrenia.

Siblings were not statistically different from controls, indicating that reduced cumulative endogenous estrogen exposure is not a marker of shared familial risk for psychotic disorder.

\section{Sex-specific findings}

All BMD measures in the femurs of female patients were significantly lower than female controls and siblings. This is in accordance with earlier findings that identified women with schizophrenia as a population that is vulnerable for reduced BMD (Meaney and O'Keane, 2007). However, the reduction 
in femoral BMD was less pronounced and with reduced significance levels (up to $p=0.086$ ) after excluding patients with prolactin-raising AP, suggesting a negative effect of prolactin-raising AP on BMD or lack of power (see below). In addition, there was no main effect of prolactin-raising AP on femoral BMD, whereas such an effect was apparent for lumbar BMD.

A population-based study concluded that schizophrenia may be an independent determinant of poor skeletal status in women, after adjusting for AP use (among other factors) (Partti et al., 2010). Maric and colleagues (Maric et al., 2005) examined BMD in the lumbar spine of 19 female patients presenting with a first episode of psychosis and healthy female controls. They found that patients had amassed significantly less bone mass than healthy controls. The femur was not examined in this study. In their sample, exposure to AP was minimal and risk factors for osteoporosis secondary to the disease status of psychotic disorder are likely less prominent in individuals presenting with a first episode of psychosis. Our findings in 16 female patients also indicated a reduction in BMD in the femur as well as in the lumbar spine (only after exclusion of affective disorder). Thus, similar to Maric and colleagues (2005), we cautiously suggest that, to the degree that BMD is a biological marker of cumulative endogenous estrogen exposure, reduced BMD may reflect diminished estrogen-induced neuroprotection and synaptic plasticity which, in turn, may place women at risk of developing a psychotic disorder. However, since this risk was not shared with siblings, it likely reflects a unique environmental experience associated with lifestyle, exogenous exposures or stochastic mechanisms. Nevertheless, if a primary role for estrogen exists, the findings indicate that it does not exclude an additional effect of prolactin-raising APs on BMD (see below under Antipsychotic medication).

In contrast to our findings in women, no association between psychotic disorder and BMD was revealed in men. Theoretically, the effects of estrogen on both brain and bone are not specific to women: androgens are aromatized focally and reach biologically effective concentrations at these tissue sites in men (Boerma et al., 2010). In support of this, beneficial effects of estrogen and its receptor modulators as adjunctive therapy for psychosis have been reported in both sexes (Kulkarni et al., 2008; Kulkarni et al., 2011; Kulkarni et al., 2010). However, in men, the availability of endogenous estrogen is entirely dependent on aromatase. Possibly, the expression and/or activity of aromatase in the brain is altered in disease states (Melcangi et al., 2011). For example, if psychotic disorder should lead to altered function of aromatase specifically in the male brain (but not in bone), cerebral estrogen levels will be low while BMD remains unaffected. If this were true, the use of BMD as an indirect marker of cerebral estrogen exposure would not be informative. In women, neuroprotective effects of estrogen may be enhanced by cyclic availability of endogenous progesterone (Azcoitia et al., 2011). Due to dysmenorrhea, women with psychotic disorder may not benefit from the synergy of both hormones, thereby enlarging the difference between groups. This lends plausibility to the hypothesis that sex-specific (patho)physiological mechanisms related to the disease state may operate.

An alternative explanation for our inconclusive findings in men may be found in the sample characteristics. The mean BMD measures of 13 male controls were rather low. Z-scores in the control group were expected to be approximately zero, as this represents the mean in the general population (corrected for sex, age and ethnicity). However, in the present sample, the mean lumbar Z-score was 
negative in male controls. In addition, all mean BMD measures of both the lumbar spine and the femur were lower in male controls than in male siblings. This may have potentially masked a true association in men and in the total group.

\section{Antipsychotic medication}

A significant association between prolactin-raising AP and BMD was found in women, but not in men. More specifically, the current use of a prolactin-raising AP was negatively associated with BMD in the lumbar spine of female patients (though not after Simes' correction). This is of interest, as our findings of reduced BMD in female patients were present in the femur, but not the lumbar spine. In the literature, there is a large body of work reporting significant negative associations between typical AP and BMD (Bilici et al., 2002; Howard et al., 2007; Kishimoto et al., 2008; Meaney et al., 2004). Conversely, several other studies failed to discover an association between BMD and APs (Howes et al., 2005; Hummer et al., 2005; Lee et al., 2010; Renn et al., 2010). Women have been described as particularly prone to AP-induced hyperprolactinemia (Kinon et al., 2003; Smith et al., 2002), although the biological substrate remains unclear. Pharmacokinetic factors, such as a higher proportion of body fat and increased cerebral blood flow that have been proposed to underlie the better treatment response to AP in women (Kolsch and Rao, 2002), may operate. Because there was a loss of effect size and statistical significance when women with prolactin-raising AP were excluded, we cannot exclude the possibility of a sex-specific susceptibility to AP-induced hyperprolactinemia in women as partial explanation for the findings. At the same time, this analysis resulted in a 37.5\% reduction in sample size and thus low statistical power to detect BMD differences at conventional alpha-level. A meta-analysis of the effects of AP on BMD has not been published but is warranted, considering the contradictory results in this clinically important matter.

\section{Methodological considerations}

A group $\times$ sex interaction was possibly not found due to an unbalanced sex distribution over the relatively small groups in combination with low mean BMD values in male controls. Thus, whether tests for interaction lacked power because of the small proportion of males in the control group, or whether there is simply no effect of group conditional on gender, remains to be elucidated in future replication studies.

All results from models restricted to the female part of the sample were upheld after Simes correction (Figure 1 and Table 4), with the exception of the repeated analyses in the smallest subgroup (exclusion of both siblings and controls with a history of affective disorder and patients with a prolactin-raising AP). It is debatable whether correction for multiple testing is appropriate in the present study. Six out of eighteen comparisons in women rendered significant findings. Given that the probability of a type I error is only $5 \%$, the number of positive findings markedly exceeds the number of false-positive findings that would have been expected by chance alone. 
Although $\mathrm{BMD}$ is an indirect measure of endogenous estrogen exposure, a clear advantage of BMD is that it reflects cumulative lifetime exposure, while the direct measurement of estrogen in serum or urine is a momentary assessment of estrogen levels. The latter would have been less informative regarding the research question under study. Nevertheless, hormone levels associated with the hypothalamus - pituitary - gonadal axis could have aided in excluding (peri)menopausal state in female patients, although subjective reports were negative and, in combination with the age range (17 - 43 years), it is unlikely that our findings, whole or in part, can be ascribed to loss of BMD secondary to menopause. Moreover, analyses were adjusted for menstrual irregularities.

The present sample is representative of the total (patient) population in our area, and confounders were corrected for. With regard to substance abuse, analyses were adjusted for the a priori hypothesized confounders of alcohol, tobacco and cannabis use. There is little evidence for an association between other (non-cannabis) drugs and BMD. In our data, there was no significant main effect of other drugs on BMD. In addition, adjustment for other drug use did not affect the pattern of the findings (results available upon request). Although best estimates of sunlight exposure were quantified, measurement of vitamin D blood levels could have been a useful alternative. In addition, serum prolactin would be a valuable objective measure. Unfortunately, these blood sample measures were not available.

The majority of publications to date focused primarily on the effects of prolactin-raising antipsychotics on BMD. To the best of our knowledge, this is the first study to investigate associations between different levels of familial risk of psychotic disorder and BMD.

Naturally, cross-sectional analyses cannot identify causal relationships. Over the follow-up, all participants have undergone a second DEXA scan. The disease course in patients and the possible transition of healthy siblings to patient status may provide further clues to the potential role of estrogen in the etiology and pathophysiology of psychotic disorders. As the literature on this topic is scarce, replication of both the negative and positive findings in a larger study population is necessary.

\section{Conclusions}

There was no evidence for low cumulative endogenous estrogen exposure (as measured by BMD), as an endophenotypic marker for psychotic disorder. BMD reduction was associated with being female and patient status, suggesting that unique environmental factors contributing to primary low estrogen levels in women may impact the risk of developing a psychotic disorder, in support of the estrogen hypothesis of schizophrenia. However, gender-specific susceptibility to the effects of prolactin-raising AP on BMD may also operate. 


\section{REFERENCES}

Abraham, G., Halbreich, U., Friedman, R.H., Josiassen, R.C., 2003a. Bone mineral density and prolactin associations in patients with chronic schizophrenia. Schizophr Res 59(1), 17-18.

Abraham, G., Paing, W.W., Kaminski, J., Joseph, A., Kohegyi, E., Josiassen, R.C., 2003b. Effects of elevated serum prolactin on bone mineral density and bone metabolism in female patients with schizophrenia: a prospective study. Am J Psychiatry 160(9), 1618-1620.

Andreasen, N.C., Flaum, M., Arndt, S., 1992. The Comprehensive Assessment of Symptoms and History (CASH). An instrument for assessing diagnosis and psychopathology. Arch Gen Psychiatry 49(8), 615-623.

APA, 2000. Diagnostic and statistical manual of mental disorders, 4th edition. American Psychiatric Association, Washington, D.C.

Azcoitia, I., Arevalo, M.A., De Nicola, A.F., Garcia-Segura, L.M., 2011. Neuroprotective actions of estradiol revisited. Trends Endocrinol Metab 22(12), 467-473.

Bab, I., Zimmer, A., Melamed, E., 2009. Cannabinoids and the skeleton: from marijuana to reversal of bone loss. Ann Med 41(8), 560-567.

Bergemann, N., Parzer, P., Mundt, C., Auler, B., 2008. High bone turnover but normal bone mineral density in women suffering from schizophrenia. Psychol Med 38(8), 1195-1201.

Bilici, M., Cakirbay, H., Guler, M., Tosun, M., Ulgen, M., Tan, U., 2002. Classical and atypical neuroleptics, and bone mineral density, in patients with schizophrenia. Int J Neurosci 112(7), 817-828.

Boerma, M.A., van der Stel, J.C., van Amelsvoort, T., Linszen, D.H., de Haan, L., 2010. Women, schizophrenia and oestrogen; neurobiological hypotheses and hormonetherapy studies. Tijdschr Psychiatr 52(4), 235-244.

Clayton, D., Hills, M., 1993. Statistical Models in Epidemiology. Oxford University Press, Oxford.

Clemons, M., Goss, P., 2001. Estrogen and the risk of breast cancer. N Engl J Med 344(4), 276-285.

Delva, N.J., Crammer, J.L., Jarzylo, S.V., Lawson, J.S., Owen, J.A., Sribney, M., Weir, B.J., Yendt, E.R., 1989. Osteopenia, pathological fractures, and increased urinary calcium excretion in schizophrenic patients with polydipsia. Biol Psychiatry 26(8), 781-793.

Goldstein, H., 1987. Multilevel Models in Educational and Social Research. Griffin, London.

Habets, P., Marcelis, M., Gronenschild, E., Drukker, M., van Os, J., 2011. Reduced cortical thickness as an outcome of differential sensitivity to environmental risks in schizophrenia. Biol Psychiatry 69(5), 487-494.

Haddad, P.M., Wieck, A., 2004. Antipsychotic-induced hyperprolactinaemia: mechanisms, clinical features and management. Drugs 64(20), 2291-2314.

Halbreich, U., 2007. Osteoporosis, schizophrenia and antipsychotics: the need for a comprehensive multifactorial evaluation. CNS Drugs 21(8), 641-657.

Howard, L., Kirkwood, G., Leese, M., 2007. Risk of hip fracture in patients with a history of schizophrenia. Br J Psychiatry 190, 129-134.

Howes, O.D., Wheeler, M.J., Meaney, A.M., O'Keane, V., Fogelman, I., Blake, G., Murray, R.M., Smith, S., 2005. Bone mineral density and its relationship to prolactin levels in patients taking antipsychotic treatment. J Clin Psychopharmacol 25(3), 259-261.

Hummer, M., Malik, P., Gasser, R.W., Hofer, A., Kemmler, G., Moncayo Naveda, R.C., Rettenbacher, M.A., Fleischhacker, W.W., 2005. Osteoporosis in patients with schizophrenia. Am J Psychiatry 162(1), 162-167.

Kinon, B.J., Gilmore, J.A., Liu, H., Halbreich, U.M., 2003. Prevalence of hyperprolactinemia in schizophrenic patients treated with conventional antipsychotic medications or risperidone. Psychoneuroendocrinology 28 Suppl 2, 55-68.

Kishimoto, T., Watanabe, K., Shimada, N., Makita, K., Yagi, G., Kashima, H., 2008. Antipsychotic-induced hyperprolactinemia inhibits the hypothalamo-pituitary-gonadal axis and reduces bone mineral density in male patients with schizophrenia. J Clin Psychiatry 69(3), 385-391. 
Kolsch, H., Rao, M.L., 2002. Neuroprotective effects of estradiol-17beta: implications for psychiatric disorders. Arch Womens Ment Health 5(3), 105-110.

Korver, N., Quee, P.J., Boos, H.B., Simons, C.J., de Haan, L., 2012. Genetic Risk and Outcome of Psychosis (GROUP), a multi site longitudinal cohort study focused on gene-environment interaction: objectives, sample characteristics, recruitment and assessment methods. Int J Methods Psychiatr Res.

Kulkarni, J., de Castella, A., Fitzgerald, P.B., Gurvich, C.T., Bailey, M., Bartholomeusz, C., Burger, H., 2008. Estrogen in severe mental illness: a potential new treatment approach. Arch Gen Psychiatry 65(8), 955-960.

Kulkarni, J., de Castella, A., Headey, B., Marston, N., Sinclair, K., Lee, S., Gurvich, C., Fitzgerald, P.B., Burger, H., 2011. Estrogens and men with schizophrenia: is there a case for adjunctive therapy? Schizophr Res 125(2-3), 278-283.

Kulkarni, J., Gurvich, C., Lee, S.J., Gilbert, H., Gavrilidis, E., de Castella, A., Berk, M., Dodd, S., Fitzgerald, P.B., Davis, S.R., 2010. Piloting the effective therapeutic dose of adjunctive selective estrogen receptor modulator treatment in postmenopausal women with schizophrenia. Psychoneuroendocrinology 35(8), 1142-1147.

Lee, T.Y., Chung, M.Y., Chung, H.K., Choi, J.H., Kim, T.Y., So, H.S., 2010. Bone density in chronic schizophrenia with longterm antipsychotic treatment: preliminary study. Psychiatry Investig 7(4), 278-284.

Lehman, D., Meyer, J.M., 2005. Decreased bone mineral density in male schizophrenia patients. Schizophr Res 76(1), 131-133.

Maric, N., Popovic, V., Jasovic-Gasic, M., Pilipovic, N., van Os, J., 2005. Cumulative exposure to estrogen and psychosis: a peak bone mass, case-control study in first-episode psychosis. Schizophr Res 73(2-3), 351-355.

Maurel, D.B., Boisseau, N., Benhamou, C.L., Jaffre, C., 2012. Alcohol and bone: review of dose effects and mechanisms. Osteoporos Int 23(1), 1-16.

Meaney, A.M., O'Keane, V., 2007. Bone mineral density changes over a year in young females with schizophrenia: relationship to medication and endocrine variables. Schizophr Res 93(1-3), 136-143.

Meaney, A.M., Smith, S., Howes, O.D., O'Brien, M., Murray, R.M., O'Keane, V., 2004. Effects of long-term prolactinraising antipsychotic medication on bone mineral density in patients with schizophrenia. Br J Psychiatry 184, 503-508.

Melcangi, R.C., Panzica, G., Garcia-Segura, L.M., 2011. Neuroactive steroids: focus on human brain. Neuroscience 191, 1-5.

Meyer, J.M., Lehman, D., 2006. Bone mineral density in male schizophrenia patients: a review. Ann Clin Psychiatry 18(1), 43-48.

Partti, K., Heliovaara, M., Impivaara, O., Perala, J., Saarni, S.l., Lonnqvist, J., Suvisaari, J.M., 2010. Skeletal status in psychotic disorders: a population-based study. Psychosom Med 72(9), 933-940.

Renn, J.H., Yang, N.P., Chou, P., 2010. Effects of plasma magnesium and prolactin on quantitative ultrasound measurements of heel bone among schizophrenic patients. BMC Musculoskelet Disord 11, 35.

Rodland, E., 2006. Simes' procedure is 'valid on average.' Biometrika 93(3), 742-746.

Simes, R., 1986. An improved Bonferroni procedure for multiple tests of significance. Biometrika 73, 751-754.

Smith, S.M., O'Keane, V., Murray, R., 2002. Sexual dysfunction in patients taking conventional antipsychotic medication. Br J Psychiatry 181, 49-55.

WHO, 1990. Composite International Diagnostic Interview (CIDI). World Health Organization, Geneva. 
a Department of Psychiatry \& Psychology, School for Mental Health and Neuroscience, EURON, Maastricht University Medical Centre, PO Box 616, 6200 MD Maastricht, the Netherlands

${ }^{b}$ Department of Nuclear Medicine, Maastricht University Medical Centre, Maastricht, the Netherlands

c Visiting Professor of Psychiatric Epidemiology, King's College London, King's Health Partners, Department of Psychosis Studies, Institute of Psychiatry, London, UK 


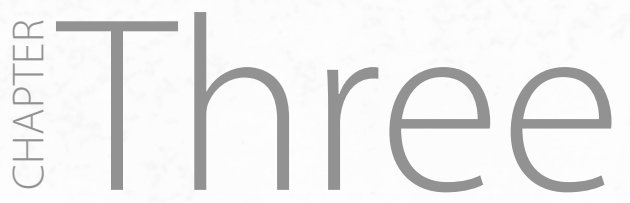

Testing the estrogen hypothesis of schizophrenia: associations between cumulative estrogen exposure and cerebral structural measures

C. van der Leeuwa ${ }^{M D}$

P. Habets ${ }^{\mathrm{a}} \mathrm{PhD}$

E. Gronenschild $\mathrm{PhD}$

P. Domen ${ }^{\mathrm{a}} \mathrm{MD}$

S. Michielse $e^{\mathrm{a} S C}$

M. van Kroonenburgh ${ }^{b}$ MD PhD

J. van Os ${ }^{\mathrm{a}, \mathrm{c}} \mathrm{MD}$ PhD

M. Marcelis ${ }^{\mathrm{a}}$ MD PhD; for G.R.O.U.P. 


\section{ABSTRACT}

Background Bone mineral density (BMD), as an indicator of cumulative estrogen exposure, may be reduced in female patients with psychotic disorder (van der Leeuw et al., 2013), possibly reflecting reduced cerebral exposure to estrogen and alterations in neuroprotective effects. To the degree that BMD is a marker of cumulative (endogenous) estrogen exposure, we hypothesized that BMD would be positively associated with cerebral grey and white matter indices.

Methods Dual X-ray absorptiometry (DEXA) and magnetic resonance (MRI) scans were acquired in fourteen female patients diagnosed with a psychotic disorder. BMD was expressed in total BMD ( $\mathrm{g} /$ $\mathrm{cm}^{2}$ ), Z- and T-scores. Cerebral cortical thickness (CT) (as indicator of grey matter status) and fractional anisotropy (FA) (as indicator of white matter integrity) were measured and served as the dependent variables in multilevel random regression models. BMD measures were the independent variables.

Results Femoral BMD measures were positively associated with $\mathrm{CT}$ at trend significance (total BMD: $B=0.266,95 \% \mathrm{Cl}:-0.019-0.552, p=0.067$; Z-score: $B=0.034,95 \%$ Cl: $0.001-0.067, p=0.046$; T-score: $B=0.034,95 \% \mathrm{Cl}: 0.000-0.068, p=0.052)$. There were no significant associations between femoral $B M D$ measures and FA.

Conclusions The data suggest that in women with psychotic disorder, alterations in the neuroprotective effect of estrogen (as measured by BMD) impact cortical grey matter, but not white matter integrity. These findings merit further investigation and, if replicated, would lend support to the estrogen hypothesis of schizophrenia. 


\section{INTRODUCTION}

Gender differences in the incidence and course of schizophrenia have been ascribed to neuroprotective effects of endogenous estrogen in females. In early adulthood, incidence rates of schizophrenia are lower in women than in men, and the age at onset of schizophrenia occurs three to four years later in women (Hafner et al., 1998; Halbreich and Kahn, 2003; Markham, 2011; Riecher-Rossler and Hafner, 1993, 2000). However, when estrogen levels decline around menopause, women display a second peak in the incidence of schizophrenia, which is absent in men (Abel et al., 2010; Hafner et al., 1998; Riecher-Rossler and Hafner, 1993). In the premenopausal period, female patients generally tend to fare better than their male counterparts, displaying a less severe course of symptoms, with a superior response to antipsychotic (AP) treatment and better social outcome (Abel et al., 2010; Hafner, 2003; Hafner et al., 1998; Markham, 2011; Salem and Kring, 1998; Seeman, 1983, 1996). Also, symptom variability during the menstrual cycle has been reported. Amelioration of symptoms is associated with a rise in estrogen and more clinical admissions take place during low estrogen phases (Huber et al., 2004; Riecher-Rossler et al., 1994; Seeman, 1996).

There are two (related) estrogen hypotheses of schizophrenia: 1) the hypoestrogenism or deficiency hypothesis which describes (chronic) gonadal dysfunction in women with schizophrenia, and 2) the protection hypothesis which states that estrogen exerts a relative protection against schizophrenia in premenopausal women (Riecher-Rossler, 2002; Riecher-Rossler and Hafner, 1993). The neuroprotective mechanism of estrogen in the human brain is complex. It includes structural effects such as the conservation of neurons, stimulation of growth and synaptogenesis, as well as effects at the receptor level, i.e. preservation of neurotransmitter receptors and modulation of neurotransmission (Azcoitia et al., 2011; Boerma et al., 2010; Brann et al., 2007; Kulkarni et al., 2012; Liu et al., 2010; McEwen et al., 2012). The dysconnectivity hypothesis of schizophrenia proposes that altered structural connectivity may represent a key pathological mechanism in schizophrenia (Friston, 1998; Konrad and Winterer, 2008). The estrogen and dysconnectivity theories are possibly linked. Recently, Peper and colleagues (2011b) reviewed the available literature reporting on the potential association between sex steroid hormones, white matter (WM) indices and functional connectivity in the human brain, and concluded that gonadal hormones appear to organize and activate structural connections within the brain. Animal studies have shown that sex steroid hormones play an essential role in myelination (Peper et al., 2011b). In humans, sex steroids have been associated with structural brain development during puberty, exerting effects on both white (Asato et al., 2010; Herting et al., 2011; Neufang et al., 2009; Peper et al., 2009; Peper et al., 2011a) and grey matter (Neufang et al., 2009; Peper et al., 2009; Peper et al., 2011a).

Other evidence for associations between estrogen and grey matter comes from the work of Goldstein and colleagues (Goldstein et al., 2001; Goldstein et al., 2002) showing disruption of the normal sexual dimorphism in schizophrenia, particularly in the cortex. In addition, hormonal fluctuations during the menstrual cycle (Pletzer et al., 2010; Protopopescu et al., 2008) and hormonal contraceptive use 
(Pletzer et al., 2010) have been associated with grey matter changes in women of childbearing age. In the context of estrogen therapy (ET) and its effect on the aging brain in postmenopausal women, both higher and lower grey matter concentrations in specific cortical areas have been suggested (Boccardi et al., 2006; Lord et al., 2010; Robertson et al., 2009). Animal models of multiple sclerosis have demonstrated prophylactic effects of estrogen with regard to grey matter atrophy (MackenzieGraham et al., 2012). Thus, disparate research fields have provided clues as to the relationship between estrogen and cerebral structure at different life stages. However, studies investigating cumulative estrogen exposure and brain structure in patients with psychotic disorder have, to the best of our knowledge, not been conducted.

Previously, we used dual X-ray absorptiometry (DEXA) to assess bone mineral density (BMD) as a marker of cumulative estrogen exposure (Clemons and Goss, 2001), and found that reduced femoral BMD was associated with being female and having a psychotic disorder, but not with familial risk for psychotic disorder. This suggests that unique environmental factors, contributing to primary low estrogen levels in women, may impact the risk of developing a psychotic disorder, in support of the estrogen (deficiency) hypothesis of schizophrenia (van der Leeuw et al., 2013).

The aim of the current study was to investigate the potential association between endogenous estrogen exposure (as indexed by BMD, corrected for exogenous estrogen exposure such as contraceptives) and grey and white matter indices in this female patient group. Using magnetic resonance imaging (MRI) and diffusion tensor imaging (DTI), cortical thickness (CT) and white matter integrity (represented by fractional anisotropy (FA)) were assessed respectively. We hypothesized that both $C T$ and FA would be positively associated with BMD, reflecting the neuroprotective effects of estrogen in schizophrenia.

\section{METHODS AND MATERIALS}

\section{Participants}

Data was collected in the context of an ongoing multicentre longitudinal study (Genetic Risk and Outcome of Psychosis, G.R.O.U.P.) in the Netherlands. In selected representative geographic areas in the Netherlands and Belgium, patients presenting consecutively at mental health services either as outpatients or inpatients were recruited for the study. Patients between the ages of 16 and 50 years, with a diagnosis of non-organic, non-affective psychosis according to DSM-IV criteria, were included (Korver et al., 2012). Sufficient command of the Dutch language was mandatory. A complete description of the recruiting protocol of the MRI sub-study is provided by Habets and colleagues (Habets et al., 2011).

As mentioned in the introduction, the aim of the current study was to investigate associations between BMD and brain measures in female patients with a psychotic disorder, as a reduction in BMD (possibly reflecting low endogenous estrogen levels) was previously found in female $(n=16)$, but not 
male $(n=46)$ patients (van der Leeuw et al., 2013). A T1-weighted structural MRI scan was obtained in 14 , and an additional DTI scan in 13 of the original 16 female patients. Thus, the present study sample comprised 14 female patients.

The sample included eight patients who were diagnosed with schizophrenia, one patient with schizoaffective disorder, four patients with a diagnosis of psychotic disorder not otherwise specified and 1 patient with a diagnosis of brief psychotic disorder. Patients were genetically unrelated. The mean illness duration was 6.6 years.

Prior to DEXA acquisition, participants were screened for the following exclusion criteria: 1) metabolic or endocrinologic disease, 2) dietary deficiency or eating disorder, 3) medication: corticosteroids, thyroxin, anti-epileptics, heparin, lithium, cytostatic agents, 4) (semi-) professional athletes, 5) polydipsia (>3 liters / day), 6) pregnancy, and 7) hormonal (infertility) treatment. Exclusion criteria for MRI constituted: 1) head injury with loss of consciousness for a duration of more than one hour, 2) meningitis of other neurological diseases that might affect brain structure or function, 3) cardiac arrhythmia requiring medical treatment, 4) severe claustrophobia, 5) (suspected) pregnancy, and 6) any metal foreign object in the body, including the presence of an intrauterine device.

\section{Measures}

Age at menarche and dysmenorrhea

Age at menarche and the occurrence of menstrual irregularity were assessed. Dysmenorrhea was specified as altered duration and/or frequency of menses or the absence of two or more menses during the previous three months. Amenorrhea was defined as the absence of menses for at least three months.

\section{Use of contraceptive drugs (exogenous estrogen exposure)}

Cumulative (lifetime) exogenous estrogen exposure in women was expressed in micrograms, as the product of daily dose and total days of use.

\section{Substance use}

Substance use was assessed using the Composite International Diagnostic Interview (CIDI). Cannabis use was assessed as the reported lifetime frequency of use. Other drug use, such as stimulants, sedatives, opiates, cocaine, PCP, psychedelics, inhalants or other (e.g. ecstasy, poppers) was assessed in the same way. Alcohol use was defined as the average number of weekly consumptions during the previous 12 months. Tobacco use was defined as the number of cigarettes per day, in the past year.

\section{Antipsychotic medication (AP) exposure}

Current AP use was classified by type: "prolactin-raising" APs, in this study comprising first-generation APs, risperidone and amisulpride; or "prolactin-sparing" APs, comprising second or third-generation 
APs with the exception of risperidone and amisulpride. Subjects who were AP-free at the time of the investigations were placed in the prolactin-sparing group.

Previous AP use was assessed retrospectively by self-report. Best estimate lifetime (cumulative) AP exposure was determined by multiplying the number of days of AP use with the daily AP dose converted to haloperidol equivalents (in milligrams), and summing all periods of use. Lifetime exposure was calculated for all APs and separately for prolactin-raising APs.

\section{DEXA acquisition and processing}

DEXA scans were acquired at Maastricht University Medical Centre with a Hologic Discovery A (Tromp Medical, Castricum, the Netherlands) (NHANES and Ethnic Reference Data). DEXA scans were performed in two anatomical areas: the lumbar spine, vertebrae $L 2$ through $L 4$; and the proximal left femur, specifically the collum, trochanter major, intertrochanteric area and Ward's triangle. BMD measures were expressed as total BMD in grams per square centimeter $\left(\mathrm{g} / \mathrm{cm}^{2}\right), Z$-scores and T-scores. The Z-score compares an individual's BMD with the mean BMD of a comparable population (with respect to gender, age and ethnicity). The T-score compares an individual's BMD to peak bone mass (PBM). Peak bone mass is the highest BMD an individual is expected to acquire during life. The T-score is used to diagnose osteopenia and osteoporosis. In the present study, the three femoral BMD measures were a priori used for further analysis, as these were reduced in female patients (van der Leeuw et al., 2013).

\section{MRI acquisition and processing}

Magnetic resonance imaging scans were obtained at Maastricht University, the Netherlands, using an Allegra syngo MR A30 (Siemens, Erlangen, Germany) operating at 3.0 Tesla. The following anatomical scan parameters were used: Modified Driven Equilibrium Fourier Transform (MDEFT) sequence; 176 slices, $1 \mathrm{~mm}$ isotropic voxel size, echo time $2.4 \mathrm{msec}$, repetition time $7.92 \mathrm{msec}$, inversion time 910 msec, flip angle 15, total acquisition time 12 min 51 sec; Magnetization Prepared Rapid Acquisition Gradient-Echo (MPRAGE; Alzheimer's Disease Neuroimaging Initiative) sequence 192 slices, $1 \mathrm{~mm}$ isotropic voxel size, echo time $2.6 \mathrm{msec}$, repetition time $2250 \mathrm{msec}$, inversion time $900 \mathrm{msec}$, flip angle $9^{\circ}$, total acquisition time $7 \mathrm{~min} 23 \mathrm{sec}$. The matrix size was $256 \times 256$ and field of view was $256 \times$ $256 \mathrm{~mm}^{2}$. The number of excitations was one. Two similar sequences were used because of a scanner update during data collection.

Microstructural anatomy was examined using diffusion tensor imaging with an echo-planar-imaging sequence (field of view $230 \mathrm{~mm}^{2}$, TR $10800 \mathrm{~ms}$, TE $84 \mathrm{~ms}$, voxel size $1.8 \times 1.8 \times 1.8 \mathrm{~mm}^{3}$, b-value $1000 \mathrm{~s} /$ $\mathrm{mm}^{2}$, noise level 40, 85 slices, no overlap). As a result of the scanner update, two DTI sequences were used: one with 76 directions (of which 4 T2-weighted (B0) and 72 diffusion-weighted (B)), and one with 81 directions ( $8 \times \mathrm{B} 0$ and $73 \times \mathrm{B})$. Total acquisition time of the DTI sequence was 15 minutes. 


\section{Cortical thickness measurement}

Scans were processed and analyzed using Freesurfer stable release v5.0.0, http://surfer.nmr.mgh. harvard.edu (Dale et al., 1999; Fischl and Dale, 2000; Fischl et al., 2002; Fischl et al., 1999; Segonne et al., 2004).

To measure CT, the cerebral cortex was parcellated into units based on gyral and sulcal structure (Desikan et al., 2006; Fischl et al., 2004). Furthermore, a variety of surface-based data was created including maps of curvature and sulcal depth. This method uses both intensity and continuity information from the entire three dimensional MR volume in segmentation and deformation procedures to produce representations of $\mathrm{CT}$, calculated as the closest distance from the grey/white matter boundary to the grey matter/CSF boundary at each vertex on the tessellated surface (Fischl and Dale, 2000). The maps are created using spatial intensity gradients across tissue classes and are not restricted to the voxel resolution of the original data, thus are capable of detecting sub-millimeter differences between groups. CT measurement procedures have been validated against histological analysis (Rosas et al., 2002) and manual measurements (Kuperberg et al., 2003; Salat et al., 2004). Individual CT values for each predefined region of interest (hereafter: ROl; adapted from the Desikan atlas (Desikan et al., 2006), 34 ROls per hemisphere) were derived by FreeSurfer and exported to STATA version 12 (StataCorp). Thus, every individual had 68 CT measurements over the predefined ROls in both hemispheres.

\section{Diffusion tensor imaging analysis}

Processing of DTI data was effectuated using tract-based spatial statistics (TBSS) v1.2 in FSL 4.1.6 (FMRIB Analysis Group, Oxford, UK, http://www.fmrib.ox.ac.uk/analysis/research/tbss). First, standard Siemens DICOM files were transformed into compressed NIFTI format using a custom built in-house software named GIANT (General Image ANalysis Tools developed by EHBMG). Raw data were corrected for head movement and eddy currents invoked during scanning. The B0 volume was skull-stripped using FSL's Brain Extraction Tool (Smith, 2002) and this served as a brain mask for all B volumes.

The next step was fitting a diffusion tensor model at each voxel using data output from the brain extraction, diffusion weighted data and gradient directions following a general linear model (FreeSurfer). After tensor fitting the process continued working on FA volumes, eroding them slightly. Nonlinear registration aligned each FA volume to $1 \times 1 \times 1 \mathrm{~mm}$ standard FMRIB58_FA space. The standard FMRIB58_FA contains a template derived from high-resolution images of 58 participants in a well-aligned population (both males and females ranging between 20 and 50 years of age) (Smith et al., 2006).

After nonlinear transformation of the FA volumes into standard space, a mean FA skeleton from all participants per group was derived. The mean FA skeleton follows the major white matter tracts in each individual participant (normalized in MNI152 space) and provides a way to compare between (groups of) participants. The final step of the processing was setting the FA threshold using visual inspection of the FA skeleton, in the present study at a level of 0.25 , to include major white matter 
tracts whilst removing small peripheral tracts that would cause excess interparticipant variability. In addition, this threshold setting avoided inclusion of regions that are likely to be composed of multiple tissue types or fiber orientations.

The Johns Hopkins University International Consortium for Brain Mapping (JHU ICBM)-DTI-81 whitematter atlas labels (Mori et al., 2008) were used to assign specific tract names. From all $38 \mathrm{JHU}$ labeled white matter tracts, mean FA values were extracted and exported to STATA.

This study was approved by the standing ethics committee. All subjects provided written informed consent in accordance with the committee's guidelines.

\section{Statistical analyses}

Conform previous work in this sample (Habets et al., 2011), datasets were transformed from a wide to a long format resulting in a hierarchical structure, with 68 regional CT measures and 38 FA values (level 1) nested in respectively 14 and 13 subjects (level 2). Given the clustering of brain measures compromising statistical independence of the observations, multilevel random regression models were fitted (Goldstein, 1987) using the XTREG command in STATA (StataCorp, version 12). Separate analyses were conducted for CT and FA. CT and FA were the dependent variables, and BMD measures were the independent variables. Although BMD measures were acquired in both the lumbar spine and the proximal femur, the current analyses were conducted a priori with the femoral BMD measures (total BMD, Z-score, T-score), as the previously reported reduction in BMD was specific for the proximal femur (van der Leeuw et al., 2013).

Analyses were adjusted for the a priori hypothesized confounders: age at scan, age at menarche, dysmenorrhea, cumulative exogenous estrogen exposure, lifetime exposure to cannabis and current use of prolactin-raising $A P(0=$ no AP or prolactin-sparing $A P, 1=$ prolactin-raising $A P)$. In separate analyses, cumulative prolactin-raising AP exposure was entered in the model replacing current prolactin-raising AP use.

Since lifetime AP exposure is a much debated potential confounder in neuroimaging research, sensitivity analyses were carried out using lifetime exposure to all AP as a covariate, instead of present and cumulative prolactin-raising AP use. 


\section{RESULTS}

\section{Descriptive analyses}

The mean age at the time of investigation was 27 years. There were six participants with dysmenorrhea. The rate of substance use in the present sample was low (see Table 1). Three women did not use AP medication at the time of the investigation. Three women used olanzapine, two women used aripiprazole, one woman used quetiapine, three women used risperidone, one woman used amisulpride, and one woman used haloperidol. Hence, five patients used a prolactin-raising AP at the time of the study. None of the patients were AP-naive and the majority (with the exception of one individual) had used a prolactin-raising AP at some point during their lives.

The use of additional psychotropic medication was minimal, and consisted of three participants with antidepressant use (of which two selective serotonin reuptake inhibitors (SSRI) and one with a serotonin and noradrenalin reuptake inhibitor (SNRI)).

The mean femoral BMD measures, as well as the mean CT and FA are shown in Table 2.

Table 1. Demographic characteristics $(n=14)$

\begin{tabular}{ll}
\hline Demographic characteristic & Mean \pm SD \\
\hline Age at scan (years) & $27.64 \pm 8.48$ \\
Age at menarche & $13.0 \pm 0.96$ \\
Dysmenorrhea (n) & 6 \\
Lifetime exogenous estrogen exposure $(\mu \mathrm{g})$ & $29923 \pm 43477$ \\
Smoking (cigarettes per day) & $6.21 \pm 8.01$ \\
Alcohol use (units per week) & $0.92 \pm 1.21$ \\
Cannabis use (number of times lifetime) & $17.71 \pm 34.60$ \\
Other drug use (number of times lifetime) & $18.71 \pm 44.97$ \\
Present exposure to PRL-raising AP (yes / no) & $5 / 9$ \\
Cumulative (lifetime) exposure to PRL-raising AP (hal. eq.) & $2262 \pm 2506$ \\
Cumulative (lifetime) exposure to all AP (hal. eq.) & $3006 \pm 3197$ \\
\hline
\end{tabular}

SD: standard deviation; PRL-raising: prolactin-raising; AP: antipsychotic medication;

hal. eq.: haloperidol equivalents (in milligrams).

Table 2. Mean femoral BMD, CT and FA

\begin{tabular}{ll}
\hline & Mean \pm SD \\
\hline Total femoral BMD $\left(\mathrm{g} / \mathrm{cm}^{2}\right)$ & $0.923 \pm 0.109$ \\
Z-score & $-0.193 \pm 0.984$ \\
T-score & $-0.257 \pm 0.971$ \\
CT $(n=14)$ & $2.553 \pm 0.375$ \\
FA $(n=13)$ & $0.576 \pm 0.086$ \\
\hline
\end{tabular}

SD: standard deviation. 


\section{Associations between BMD and CT}

BMD measures of the femur were positively associated with $C T$ when correcting for current exposure to a prolactin-raising AP (total BMD: $B=0.266,95 \% C l$ : $-0.019-0.552, p=0.067$; Z-score: $B=0.034,95 \%$ Cl: $0.001-0.067, p=0.046 ;$ T-score: $B=0.034,95 \% \mathrm{Cl}: 0.000-0.068, p=0.052$ ) (Table 3). When correcting for lifetime exposure to prolactin-raising AP, the positive association was strengthened (total BMD: $B=0.429,95 \%$ Cl: $0.104-0.753, p=0.010$; Z-score: $B=0.049,95 \%$ Cl: $0.012-0.085, p=0.009 ;$ T-score: $\mathrm{B}=0.048,95 \% \mathrm{Cl}: 0.011-0.086, \mathrm{p}=0.011$ ) (Table 3). As described under the heading Statistical Analysis, a sensitivity analysis was carried out with lifetime exposure to all AP replacing present or cumulative exposure to prolactin-raising AP as a covariate. The positive association between femoral BMD measures and CT remained (total BMD: $B=0.390,95 \% C l$ : $0.091-0.689, p=0.011$; Z-score: $B=0.046,95 \%$ Cl: $0.012-0.080, p=0.008$; T-score: $B=0.046,95 \%$ Cl: $0.011-0.081, p=0.010$ ).

\section{Associations between BMD and FA}

There were no significant associations between femoral BMD measures and FA (Table 3). Substitution of either covariate pertaining to prolactin-raising AP use with lifetime exposure to all AP did not alter the pattern of findings.

Table 3. Associations between femoral BMD and structural brain measures

\begin{tabular}{|c|c|c|c|}
\hline & Total BMD & Z-score & T-score \\
\hline & B (95\% Cl), p-value & B $(95 \%$ Cl), p-value & B (95\% Cl), p-value \\
\hline \multicolumn{4}{|l|}{$C T(n=14)$} \\
\hline \multicolumn{4}{|l|}{ Correction for: } \\
\hline Present exposure PRL-raising AP & $\begin{array}{l}0.266(-0.019-0.552) \\
0.067\end{array}$ & $\begin{array}{l}0.034(0.001-0.067) \\
0.046\end{array}$ & $\begin{array}{l}0.034(0.000-0.068) \\
0.052\end{array}$ \\
\hline Cumulative exposure PRL-raising AP & $\begin{array}{l}0.429(0.104-0.753) \\
0.010\end{array}$ & $\begin{array}{l}0.049(0.012-0.085) \\
0.009\end{array}$ & $\begin{array}{l}0.048(0.011-0.086) \\
0.011\end{array}$ \\
\hline \multicolumn{4}{|l|}{ FA $(n=13)$} \\
\hline \multicolumn{4}{|l|}{ Correction for: } \\
\hline Present exposure PRL-raising AP & $\begin{array}{l}0.002(-0.134-0.138) \\
0.973\end{array}$ & $\begin{array}{l}0.000(-0.015-0.015) \\
0.993\end{array}$ & $\begin{array}{l}0.000(-0.015-0.015) \\
0.992\end{array}$ \\
\hline Cumulative exposure PRL-raising AP & $\begin{array}{l}0.012(-0.287-0.311) \\
0.937\end{array}$ & $\begin{array}{l}-0.001(-0.027-0.026) \\
0.970\end{array}$ & $\begin{array}{l}-0.001(-0.028-0.026) \\
0.941\end{array}$ \\
\hline
\end{tabular}

B's represent the regression coefficient of the multilevel regression analyses.

$\mathrm{Cl}$ : confidence interval; PRL-raising AP: prolactin-raising antipsychotic medication. 


\section{DISCUSSION}

The present study examined, for the first time - to the best of our knowledge, associations between cumulative endogenous estrogen exposure (to the extent that it is reflected by femoral BMD) and brain structure in psychotic disorder. Our findings tentatively suggest that higher cumulative exposure to estrogen is associated with increased cerebral cortical thickness. There was no evidence for a significant association between cumulative estrogen exposure and microstructural white matter integrity.

\section{Findings}

\section{Cumulative endogenous estrogen exposure and CT}

Our findings showed positive associations between BMD measures and CT in female patients with a psychotic disorder. In other words, higher cumulative estrogen levels may be associated with increased $\mathrm{CT}$, or lower estrogen levels may be associated with reduced $\mathrm{CT}$. Assuming the association is true, a deficiency in estrogen exposure may impact grey matter, which may be reversed by higher levels of estrogen that may induce or activate neuroprotective mechanisms. The results thus fit both the estrogen deficiency and protection hypothesis.

Accumulating clinical research has demonstrated emerging treatment perspectives of estrogen therapy (ET) and selective estrogen receptor modulators (SERMs) as adjunctive therapy (Kulkarni et al., 2010; Kulkarni et al., 2012). Following an earlier, inconclusive Cochrane review (Chua et al., 2005), a quantitative 2012 review suggests that the use of estrogen as adjunctive treatment for schizophrenia could be effective in women (Begemann et al., 2012). Though this indicates an association between hypoestrogenism and psychotic disorders in women, the question remains whether hypoestrogenism is a cause or consequence of the disease (Aston et al., 2010; Markham, 2011), or its treatment.

Interpretation of the current study findings is complicated by the cross-sectional nature of the data, as well as the fact that no previous studies have been conducted combining (markers of) estrogen levels and structural brain alterations in psychiatrically ill individuals. Nevertheless, disrupted sexual dimorphisms in the cortex of patients with schizophrenia as demonstrated by MRI (Goldstein et al., 2002) were reported over a decade ago, which helped refuel the debate about sex differences in schizophrenia. It seems biologically plausible that estrogen, through its impact on brain morphology, contributes to the risk for - and the sex differences in - psychotic disorder. Indirect support for this comes from a study in healthy postmenopausal women, showing beneficial influence of ET on the cortical cerebral surface (Boccardi et al., 2006). However, other groups failed to discover an association between ET and total grey matter (Ha et al., 2007; Low et al., 2006), or reported a negative association (Greenberg et al., 2006). During adolescence, higher estrogen levels are related to an overall decrease in cortical grey matter (Peper et al., 2009; Peper et al., 2011a), demonstrating that the organizing and structural effects of estrogen may be age-dependent and influenced by other factors. 


\section{Cumulative endogenous estrogen exposure and FA}

We did not find an association between BMD and FA. The literature on associations between estrogen and cerebral white matter is limited. In the developing brain, white matter increase is minimal in girls compared to boys, and white matter maturation may be primarily under the influence of luteinizing hormone (which is positively associated with white matter in puberty) (Peper et al., 2011a). In one study, estrogen was positively related to FA in boys, yet negatively related to FA in girls (Herting et al., 2011). One reason for the absence of an association between estrogen and FA in the current study, may be that other gonadal hormones constitute the principal hormonal effect on white matter, e.g. progesterone elicits the production of myelin and oligodendrocytes (Peper et al., 2011 b). Results from studies investigating the effect of ET on white matter in the aging brain are inconsistent. Low and colleagues (Low et al., 2006) reported inconclusive findings with regard to the effect of ET on white matter volume, while $\mathrm{Ha}$ (Ha et al., 2007) found significantly greater white matter volumes in estrogen users.

\section{Influence of AP on brain measures and BMD}

AP exposure may be associated with both brain measures and BMD and may thus act as a potential confounder. With regard to the effect on brain structure, some studies found, in fairly large samples, no effect of duration of AP use or illness duration on white matter microstructure (Kanaan et al., 2009) or grey matter (Leung et al., 2011). In contrast, a large prospective study (van Haren et al., 2011) found that cortical thinning is a progressive process and is in part mediated by the use of AP. A recent metareview (Shepherd et al., 2012) reported that structural brain changes (including cortical volume) may be affected by AP, though the effect of AP exposure may itself be confounded by disease progression (Ho et al., 2011).

In the current study, we initially corrected for the present use of a prolactin-raising AP or cumulative exposure to prolactin-raising AP, in line with our hypothesis that cerebral estrogen exposure may be affected by hyperprolactinemia-induced hypogonadism secondary to prolactin-raising AP. When using lifetime exposure to all AP instead of current or lifetime prolactin-raising AP use in the analyses, the positive associations between BMD and CT remained, and the absence of associations between BMD and FA was confirmed.

With regard to the relation between $A P$ and $B M D$, the majority of studies are suggestive of a negative association (Crews and Howes, 2012). In our original BMD study ( $n=16)$, we found a negative main effect of prolactin-raising AP on lumbar BMD, but not femoral BMD (van der Leeuw et al., 2013). In addition, due to a reduction in sample size (and concomitant effect size reduction) when excluding female patients who used prolactin-raising APs, we were unable to exclude the possibility of AP exposure as a partial explanation for reduced BMD in female patients. However, the specific association between BMD and CT (and not FA) in the present study, corrected for present and lifetime prolactin-raising AP use and cumulative exposure to all AP, is at least suggestive of an independent cerebral effect of primary estrogen reduction in female patients with psychotic disorder. 


\section{Methodological considerations}

Certain limitations in our study design need to be acknowledged.

First, bone mineral density was used as an indirect indicator of cumulative (endogenous) estrogen exposure and has disadvantages because it is influenced by many hormonal and non-hormonal factors and should thus be interpreted as a proxy marker. Nevertheless, the most important potential confounding factors were addressed. A clear advantage of BMD is that it reflects cumulative exposure to estrogen, while the direct measurement of estrogen in serum or urine is a momentary assessment. Although we could not consider the influence of the phase of the menstrual cycle on brain morphology as recommended by Pletzer (Pletzer et al., 2010), we adjusted for several hormonal factors such as age at menarche, dysmenorrhea, lifetime contraceptive use and current and lifetime prolactin-raising AP use.

Second, the sample size of the study was small, suggesting that our findings are best interpreted as hypothesis-generating. The sample size did not preclude the detection of positive findings in CT. However, it is possible that our study lacked power to detect an effect in FA. To date, other neuroimaging studies investigating estrogen effects on brain morphology in schizophrenia have not been performed and the present hypothesis-generating study therefore requires replication with larger sample sizes, to corroborate or challenge the results. The presented cross-sectional data is part of a longitudinal study. Follow-up data may provide further clues to the relationship between lifetime estrogen exposure and brain alteration (over time) in psychotic disorder.

Third, it is possible that the effects of estrogen are region-specific. The present study was not powered to examine whether the impact of BMD varied with specific CT or FA regions (i.e. BMD $\times$ region interaction). To discern regions of interest, an experimental treatment study using ET or SERMs may offer additional insights.

Fourth, our study is limited by the fact that our subjects were not first-episode AP-naïve patients. Inherent to the inclusion criteria of the GROUP study, individuals with a relatively recent onset of psychotic disorder were included (mean illness duration of 6.6 years). Thus, by implication, none of our patients were AP-naïve and the majority had used a prolactin-raising AP at some point in their lives. Nevertheless, the pattern of results remained consistent after extensive correction for AP use. Ideally, replication should be performed in a sample that has been exposed to neither AP nor contraconceptive drugs.

\section{Conclusions}

Reduced BMD (reflecting low cumulative estrogen levels) in female patients with a psychotic disorder may be associated with decreased cortical thickness, suggesting that a diminished neuroprotective effect of estrogen may contribute to cerebral grey matter alterations, indirectly supporting both aspects (deficiency and protection) of the estrogen hypothesis of schizophrenia. There was no evidence for an association between BMD and white matter integrity. 


\section{REFERENCES}

Abel, K.M., Drake, R., Goldstein, J.M., 2010. Sex differences in schizophrenia. Int Rev Psychiatry 22(5), 417-428.

Asato, M.R., Terwilliger, R., Woo, J., Luna, B., 2010. White matter development in adolescence: a DTI study. Cereb Cortex 20(9), 2122-2131.

Aston, J., Rechsteiner, E., Bull, N., Borgwardt, S., Gschwandtner, U., Riecher-Rossler, A., 2010. Hyperprolactinaemia in early psychosis-not only due to antipsychotics. Prog Neuropsychopharmacol Biol Psychiatry 34(7), 1342-1344.

Azcoitia, I., Arevalo, M.A., De Nicola, A.F., Garcia-Segura, L.M., 2011. Neuroprotective actions of estradiol revisited. Trends Endocrinol Metab 22(12), 467-473.

Begemann, M.J., Dekker, C.F., van Lunenburg, M., Sommer, I.E., 2012. Estrogen augmentation in schizophrenia: A quantitative review of current evidence. Schizophr Res 141(2-3), 179-184.

Boccardi, M., Ghidoni, R., Govoni, S., Testa, C., Benussi, L., Bonetti, M., Binetti, G., Frisoni, G.B., 2006. Effects of hormone therapy on brain morphology of healthy postmenopausal women: a Voxel-based morphometry study. Menopause 13(4), 584-591.

Boerma, M.A., van der Stel, J.C., van Amelsvoort, T., Linszen, D.H., de Haan, L., 2010. [Women, schizophrenia and oestrogen; neurobiological hypotheses and hormonetherapy studies].Tijdschrift voor psychiatrie 52(4), 235-244.

Brann, D.W., Dhandapani, K., Wakade, C., Mahesh, V.B., Khan, M.M., 2007. Neurotrophic and neuroprotective actions of estrogen: basic mechanisms and clinical implications. Steroids 72(5), 381-405.

Chua, W.L., de Izquierdo, S.A., Kulkarni, J., Mortimer, A., 2005. Estrogen for schizophrenia. Cochrane Database Syst $\operatorname{Rev}(4), \operatorname{CD} 004719$.

Clemons, M., Goss, P., 2001. Estrogen and the risk of breast cancer. N Engl J Med 344(4), 276-285.

Crews, M.P., Howes, O.D., 2012. Is antipsychotic treatment linked to low bone mineral density and osteoporosis? A review of the evidence and the clinical implications. Human psychopharmacology 27(1), 15-23.

Dale, A.M., Fischl, B., Sereno, M.I., 1999. Cortical surface-based analysis. I. Segmentation and surface reconstruction. Neuroimage 9(2), 179-194.

Desikan, R.S., Segonne, F., Fischl, B., Quinn, B.T., Dickerson, B.C., Blacker, D., Buckner, R.L., Dale, A.M., Maguire, R.P., Hyman, B.T., Albert, M.S., Killiany, R.J., 2006. An automated labeling system for subdividing the human cerebral cortex on MRI scans into gyral based regions of interest. Neuroimage 31(3), 968-980.

Fischl, B., Dale, A.M., 2000. Measuring the thickness of the human cerebral cortex from magnetic resonance images. Proc Natl Acad Sci U S A 97(20), 11050-11055.

Fischl, B., Salat, D.H., Busa, E., Albert, M., Dieterich, M., Haselgrove, C., van der Kouwe, A., Killiany, R., Kennedy, D. Klaveness, S., Montillo, A., Makris, N., Rosen, B., Dale, A.M., 2002. Whole brain segmentation: automated labeling of neuroanatomical structures in the human brain. Neuron 33(3), 341-355.

Fischl, B., Sereno, M.I., Dale, A.M., 1999. Cortical surface-based analysis. II: Inflation, flattening, and a surface-based coordinate system. Neuroimage 9(2), 195-207.

Fischl, B., van der Kouwe, A., Destrieux, C., Halgren, E., Segonne, F., Salat, D.H., Busa, E., Seidman, L.J., Goldstein, J., Kennedy, D., Caviness, V., Makris, N., Rosen, B., Dale, A.M., 2004. Automatically parcellating the human cerebral cortex. Cereb Cortex 14(1), 11-22.

Friston, K.J., 1998. The disconnection hypothesis. Schizophr Res 30(2), 115-125.

Goldstein, H., 1987. Multilevel Models in Educational and Social Research. Griffin, London.

Goldstein, J.M., Seidman, L.J., O’Brien, L.M., Horton, N.J., Kennedy, D.N., Makris, N., Caviness, V.S., Jr., Faraone, S.V., Tsuang, M.T., 2002. Impact of normal sexual dimorphisms on sex differences in structural brain abnormalities in schizophrenia assessed by magnetic resonance imaging. Arch Gen Psychiatry 59(2), 154-164.

Greenberg, D.L., Payne, M.E., MacFall, J.R., Provenzale, J.M., Steffens, D.C., Krishnan, R.R., 2006. Differences in brain volumes among males and female hormone-therapy users and nonusers. Psychiatry Res 147(2-3), 127-134. 
Ha, D.M., Xu, J., Janowsky, J.S., 2007. Preliminary evidence that long-term estrogen use reduces white matter loss in aging. Neurobiol Aging 28(12), 1936-1940.

Habets, P., Marcelis, M., Gronenschild, E., Drukker, M., van Os, J., 2011. Reduced cortical thickness as an outcome of differential sensitivity to environmental risks in schizophrenia. Biol Psychiatry 69(5), 487-494.

Hafner, H., 2003. Gender differences in schizophrenia. Psychoneuroendocrinology 28 Suppl 2, 17-54.

Hafner, H., an der Heiden, W., Behrens, S., Gattaz, W.F., Hambrecht, M., Loffler, W., Maurer, K., Munk-Jorgensen, P., Nowotny, B., Riecher-Rossler, A., Stein, A., 1998. Causes and consequences of the gender difference in age at onset of schizophrenia. Schizophr Bull 24(1), 99-113.

Halbreich, U., Kahn, L.S., 2003. Hormonal aspects of schizophrenias: an overview. Psychoneuroendocrinology 28 Suppl 2, 1-16.

Herting, M.M., Maxwell, E.C., Irvine, C., Nagel, B.J., 2011. The Impact of Sex, Puberty, and Hormones on White Matter Microstructure in Adolescents. Cereb Cortex.

Ho, B.C., Andreasen, N.C., Ziebell, S., Pierson, R., Magnotta, V., 2011. Long-term antipsychotic treatment and brain volumes: a longitudinal study of first-episode schizophrenia. Arch Gen Psychiatry 68(2), 128-137.

Huber, T.J., Borsutzky, M., Schneider, U., Emrich, H.M., 2004. Psychotic disorders and gonadal function: evidence supporting the oestrogen hypothesis. Acta Psychiatr Scand 109(4), 269-274.

Kanaan, R., Barker, G., Brammer, M., Giampietro, V., Shergill, S., Woolley, J., Picchioni, M., Toulopoulou, T., McGuire, P., 2009. White matter microstructure in schizophrenia: effects of disorder, duration and medication. $\mathrm{Br} J$ Psychiatry 194(3), 236-242.

Konrad, A., Winterer, G., 2008. Disturbed structural connectivity in schizophrenia primary factor in pathology or epiphenomenon? Schizophr Bull 34(1), 72-92.

Korver, N., Quee, P.J., Boos, H.B., Simons, C.J., de Haan, L., 2012. Genetic Risk and Outcome of Psychosis (GROUP), a multi site longitudinal cohort study focused on gene-environment interaction: objectives, sample characteristics, recruitment and assessment methods. Int J Methods Psychiatr Res 21(3), 205-221.

Kulkarni, J., Gurvich, C., Lee, S.J., Gilbert, H., Gavrilidis, E., de Castella, A., Berk, M., Dodd, S., Fitzgerald, P.B., Davis, S.R., 2010. Piloting the effective therapeutic dose of adjunctive selective estrogen receptor modulator treatment in postmenopausal women with schizophrenia. Psychoneuroendocrinology 35(8), 1142-1147.

Kulkarni, J., Hayes, E., Gavrilidis, E., 2012. Hormones and schizophrenia. Curr Opin Psychiatry 25(2), 89-95.

Kuperberg, G.R., Broome, M.R., McGuire, P.K., David, A.S., Eddy, M., Ozawa, F., Goff, D., West, W.C., Williams, S.C., van der Kouwe, A.J., Salat, D.H., Dale, A.M., Fischl, B., 2003. Regionally localized thinning of the cerebral cortex in schizophrenia. Arch Gen Psychiatry 60(9), 878-888.

Leung, M., Cheung, C., Yu, K., Yip, B., Sham, P., Li, Q., Chua, S., McAlonan, G., 2011. Gray matter in first-episode schizophrenia before and after antipsychotic drug treatment. Anatomical likelihood estimation meta-analyses with sample size weighting. Schizophr Bull 37(1), 199-211.

Liu, M., Kelley, M.H., Herson, P.S., Hurn, P.D., 2010. Neuroprotection of sex steroids. Minerva Endocrinol 35(2), $127-143$.

Lord, C., Engert, V., Lupien, S.J., Pruessner, J.C., 2010. Effect of sex and estrogen therapy on the aging brain: a voxelbased morphometry study. Menopause 17(4), 846-851.

Low, L.F., Anstey, K.J., Maller, J., Kumar, R., Wen, W., Lux, O., Salonikas, C., Naidoo, D., Sachdev, P., 2006. Hormone replacement therapy, brain volumes and white matter in postmenopausal women aged 60-64 years. Neuroreport 17(1), 101-104.

Mackenzie-Graham, A.J., Rinek, G.A., Avedisian, A., Morales, L.B., Umeda, E., Boulat, B., Jacobs, R.E., Toga, A.W., Voskuhl, R.R., 2012. Estrogen treatment prevents gray matter atrophy in experimental autoimmune encephalomyelitis. J Neurosci Res.

Markham, J.A., 2011. Sex steroids and schizophrenia. Rev Endocr Metab Disord. 
McEwen, B.S., Akama, K.T., Spencer-Segal, J.L., Milner, T.A., Waters, E.M., 2012. Estrogen effects on the brain: actions beyond the hypothalamus via novel mechanisms. Behav Neurosci 126(1), 4-16.

Mori, S., Oishi, K., Jiang, H., Jiang, L., Li, X., Akhter, K., Hua, K., Faria, A.V., Mahmood, A., Woods, R., Toga, A.W., Pike, G.B., Neto, P.R., Evans, A., Zhang, J., Huang, H., Miller, M.I., van Zijl, P., Mazziotta, J., 2008. Stereotaxic white matter atlas based on diffusion tensor imaging in an ICBM template. Neurolmage 40(2), 570-582.

Neufang, S., Specht, K., Hausmann, M., Gunturkun, O., Herpertz-Dahlmann, B., Fink, G.R., Konrad, K., 2009. Sex differences and the impact of steroid hormones on the developing human brain. Cereb Cortex 19(2), 464-473.

Peper, J.S., Brouwer, R.M., Schnack, H.G., van Baal, G.C., van Leeuwen, M., van den Berg, S.M., Delemarre-Van de Waal, H.A., Boomsma, D.I., Kahn, R.S., Hulshoff Pol, H.E., 2009. Sex steroids and brain structure in pubertal boys and girls. Psychoneuroendocrinology 34(3), 332-342.

Peper, J.S., Hulshoff Pol, H.E., Crone, E.A., van Honk, J., 2011a. Sex steroids and brain structure in pubertal boys and girls: a mini-review of neuroimaging studies. Neuroscience 191, 28-37.

Peper, J.S., van den Heuvel, M.P., Mandl, R.C., Pol, H.E., van Honk, J., 2011 b. Sex steroids and connectivity in the human brain: a review of neuroimaging studies. Psychoneuroendocrinology 36(8), 1101-1113.

Pletzer, B., Kronbichler, M., Aichhorn, M., Bergmann, J., Ladurner, G., Kerschbaum, H.H., 2010. Menstrual cycle and hormonal contraceptive use modulate human brain structure. Brain Res 1348, 55-62.

Protopopescu, X., Butler, T., Pan, H., Root, J., Altemus, M., Polanecsky, M., McEwen, B., Silbersweig, D., Stern, E., 2008. Hippocampal structural changes across the menstrual cycle. Hippocampus 18(10), 985-988.

Riecher-Rossler, A., 2002. Oestrogen effects in schizophrenia and their potential therapeutic implications--review. Arch Womens Ment Health 5(3), 111-118.

Riecher-Rossler, A., Hafner, H., 1993. Schizophrenia and oestrogens--is there an association? Eur Arch Psychiatry Clin Neurosci 242(6), 323-328.

Riecher-Rossler, A., Hafner, H., 2000. Gender aspects in schizophrenia: bridging the border between social and biological psychiatry. Acta Psychiatr Scand Suppl(407), 58-62.

Riecher-Rossler, A., Hafner, H., Stumbaum, M., Maurer, K., Schmidt, R., 1994. Can estradiol modulate schizophrenic symptomatology? Schizophr Bull 20(1), 203-214

Robertson, D., Craig, M., van Amelsvoort, T., Daly, E., Moore, C., Simmons, A., Whitehead, M., Morris, R., Murphy, D., 2009. Effects of estrogen therapy on age-related differences in gray matter concentration. Climacteric 12(4), 301-309.

Rosas, H.D., Liu, A.K., Hersch, S., Glessner, M., Ferrante, R.J., Salat, D.H., van der Kouwe, A., Jenkins, B.G., Dale, A.M., Fischl, B., 2002. Regional and progressive thinning of the cortical ribbon in Huntington's disease. Neurology 58(5), 695-701.

Salat, D.H., Buckner, R.L., Snyder, A.Z., Greve, D.N., Desikan, R.S., Busa, E., Morris, J.C., Dale, A.M., Fischl, B., 2004. Thinning of the cerebral cortex in aging. Cereb Cortex 14(7), 721-730.

Salem, J.E., Kring, A.M., 1998. The role of gender differences in the reduction of etiologic heterogeneity in schizophrenia. Clin Psychol Rev 18(7), 795-819.

Seeman, M.V., 1983. Interaction of sex, age, and neuroleptic dose. Compr Psychiatry 24(2), 125-128.

Seeman, M.V., 1996. The role of estrogen in schizophrenia. J Psychiatry Neurosci 21(2), 123-127.

Segonne, F., Dale, A.M., Busa, E., Glessner, M., Salat, D., Hahn, H.K., Fischl, B., 2004. A hybrid approach to the skull stripping problem in MRI. Neuroimage 22(3), 1060-1075.

Shepherd, A.M., Laurens, K.R., Matheson, S.L., Carr, V.J., Green, M.J., 2012. Systematic meta-review and quality assessment of the structural brain alterations in schizophrenia. Neurosci Biobehav Rev 36(4), 1342-1356.

Smith, S.M., 2002. Fast robust automated brain extraction. Human brain mapping 17(3), 143-155.

Smith, S.M., Jenkinson, M., Johansen-Berg, H., Rueckert, D., Nichols, T.E., Mackay, C.E., Watkins, K.E., Ciccarelli, O., Cader, M.Z., Matthews, P.M., Behrens, T.E., 2006. Tract-based spatial statistics: voxelwise analysis of multi-subject diffusion data. Neurolmage 31(4), 1487-1505. 
van der Leeuw, C., Habets, P., Domen, P., van Kroonenburgh, M., van Os, J., Marcelis, M., 2013. Bone mineral density as a marker of cumulative endogenous estrogen exposure: Relationship to background genetic risk of psychotic disorder. Schizophrenia research 143(1), 25-31.

van Haren, N.E., Schnack, H.G., Cahn, W., van den Heuvel, M.P., Lepage, C., Collins, L., Evans, A.C., Hulshoff Pol, H.E., Kahn, R.S., 2011. Changes in cortical thickness during the course of illness in schizophrenia. Arch Gen Psychiatry 68(9), 871-880. 
a Department of Psychiatry \& Psychology, School for Mental Health and Neuroscience, EURON, Maastricht University Medical Centre, Maastricht, the Netherlands.

b Department of Radiology \& Nuclear Medicine, Maastricht University Medical Centre, Maastricht, the Netherlands.

c Visiting Professor of Psychiatric Epidemiology, Department of Psychosis Studies, Institute of Psychiatry, King's College London, UK..--

d Institute for Mental Health Care Eindhoven (GGzE), Eindhoven, the Netherlands 


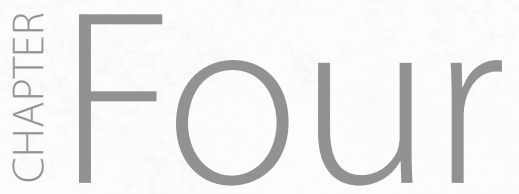

Bone mineral density as a marker of cumulative estrogen exposure in psychotic disorder:

a 3 year follow-up study

C. van der Leeuw MDa

S. Peeters MSC $\mathrm{C}^{\mathrm{a}}$

P. Domen $\mathrm{MD}^{\mathrm{a}}$

M. van Kroonenburgh MD PhD

J. van Os MD PhDa,

M. Marcelis MD PhDa,; for G.R.O.U.P. 


\section{ABSTRACT}

Background Altered estrogen-induced neuroprotection has been implicated in the etiology of psychotic disorders. Using bone mineral density (BMD) as a marker of lifetime estrogen exposure, a longitudinal family study was conducted to discriminate between etiological mechanisms and secondary effects of disease and treatment.

Methods Dual X-ray absorptiometry (DEXA) scans were acquired twice, with an interval of 3 years, in 30 patients with psychotic disorder (male (M)/female (F): 24/6, mean age of 32 years at second DEXA), 44 non-psychotic siblings of patients with a psychotic disorder (M/F: 26/18, mean age 32) and 27 controls (M/F: 7/20, mean age 35). Bone mineral density (BMD) was measured in the lumbar spine and proximal femur. Associations between group and BMD changes were investigated with multilevel random regression analyses. The effect of prolactin (PRL) -raising antipsychotics (AP) was evaluated.

Results (Increased risk of) psychotic disorder was not associated with disproportionate BMD loss over a three year period. Instead, femoral BMD measures appeared to decrease less in the patient versus control comparison (total BMD: $B=0.026,95 \% \mathrm{Cl} 0.002$ to $0.050, p=0.037$; Z-score: $B=0.224,95 \% \mathrm{Cl}$ 0.035 to $0.412, p=0.020$; and T-score: $B=0.193,95 \% \mathrm{Cl} 0.003$ to $0.382, p=0.046$ ). Current or past use of a prolactin-raising AP was not associated with BMD changes.

Conclusions In this small longitudinal study, there was no evidence of ongoing estrogen deficiency in psychotic disorder as there was no excessive BMD loss over a 3-year period in patients using AP. No proof of effects of disease-related factors or PRL-raising AP on BMD was found. 


\section{INTRODUCTION}

A recent review concluded that "schizophrenia is associated with reduced bone mineral density (BMD) and fracture risk" (Kishimoto et al., 2012), possibly resulting from a high prevalence of risk factors for osteoporosis in this population (Halbreich, 2007). Another recent review reported that BMD is reduced in patients who use AP medication (Crews and Howes, 2012), with first generation and other prolactin-raising APs particularly implicated (Abraham et al., 2003; Bilici et al., 2002; Haddad and Wieck, 2004; Howard et al., 2007; Meaney and O'Keane, 2007; Meaney et al., 2004). Stubbs and colleagues conducted the first meta-analysis examining low bone mass in schizophrenia and concluded that patients with schizophrenia were more than twice as likely to have osteoporosis in comparison to age and sex matched controls (Stubbs et al., 2014).

Interestingly, schizophrenia has been proposed as an independent determinant of skeletal status, irrespective of AP use and other secondary factors (Partti et al., 2010). Estrogen is an important factor in bone metabolism, demonstrated by the fact that osteoporosis risk rises after menopause. Taking this a step further, BMD may be an intermediate marker of cumulative estrogen exposure (Clemons and Goss, 2001) and may reflect alterations in the neuroprotective estrogenic impact in the brain (Maric et al., 2005). Maric and colleagues demonstrated that female patients, presenting with a first episode of psychosis, had amassed significantly less bone mass than healthy controls. This may be indicative of a primary role for estrogen in the etiology of schizophrenia, as the confounding influence of medication and lifestyle factors is presumed to be minimal at such an early stage of disease. Theoretically, the impact of estrogen on both brain and bone are not specific to women: androgens are aromatized focally and reach biologically effective concentrations at these tissue sites in men (Boerma et al., 2010). Previously, we assessed BMD as a marker of lifetime estrogen exposure, in relation to background genetic risk of psychotic disorder (van der Leeuw et al., 2013a). BMD was unaltered in healthy siblings, though it was reduced in the femur of female, but not male, patients with psychotic disorder. While the use of prolactin-raising AP could not be dismissed as a possible contributing factor, there was some evidence to suggest that reduced BMD in female patients represents diminished estrogen-induced neuroprotection, in support of the estrogen hypothesis of schizophrenia. Examining associations between BMD and neuroimaging data obtained in the same group of female patients enabled us to further examine this finding. There was evidence for a positive association between BMD and cerebral cortical thickness (CT) (van der Leeuw et al., 2013b), suggesting that low cumulative estrogen levels may be accompanied by reduced neuroprotection.

It remains unclear whether hypoestrogenism is a cause and / or result of psychotic disorder (Markham, 2012). It is also unclear how hyperprolactinemia causes reduced BMD. Both indirect (in the presence of hypogonadism) and direct pathways (in the absence of hypogonadism) have been proposed (Crews and Howes, 2012). Although four longitudinal studies have been performed examining BMD alterations in relation to prolactin-raising AP (Abraham et al., 2003; Meaney and O'Keane, 2007; Takahashi et al., 2013; Wang et al., 2014), prospective studies designed to discriminate between 
etiological mechanisms and secondary effects of disease and treatment have not been conducted. After a follow-up period of 3 years, we reassessed BMD in the original sample (van der Leeuw et al., 2013a). We hypothesized that patients would show a disproportionate decline in BMD compared to siblings and controls, reflecting primary factors associated with the disorder (continuous low estrogen levels). Excessive BMD loss in patients and siblings compared to patients would lend support to hypoestrogenism as an endophenotype of psychotic disorder, not influenced by disease-related factors. Though no evidence of reduced BMD in siblings was found at baseline, we wished to confirm this at follow-up. Furthermore, BMD changes in siblings would be of particular interest if siblings transitioned to the patient group during the three year interval. Potential adverse disease-related effects were monitored in patients (including lifestyle factors and AP use), as they may also contribute to reduced BMD (Kishimoto et al., 2012).

\section{METHODS AND MATERIALS}

\section{Participants}

Dual X-ray absorptiometry (DEXA) scans were conducted in the context of a longitudinal Dutch study Genetic Risk and Outcome of Psychosis (GROUP) (Korver et al., 2012; van der Leeuw et al., 2013a). The study population consisted of patients with a diagnosis of non-organic, non-affective psychosis, their non-psychotic siblings and a control group. Diagnosis was based on DSM-IV criteria (APA, 2000), assessed with the Comprehensive Assessment of Symptoms and History (CASH) interview (Andreasen et al., 1992). The CASH was also used to confirm the absence of a diagnosis of non-affective psychosis in the siblings, and absence of a diagnosis of any psychotic disorder in the control participants. For the control participants, the occurrence of any psychotic disorder in either the participant or any first-degree family member, assessed using the Family Interview for Genetic Studies, constituted an exclusion criterion. Exclusion criteria included: 1) metabolic or endocrine disease, 2) dietary deficiency or eating disorder, 3) medication use: corticosteroids, thyroxin, anti-epileptic drugs, heparin, lithium, cytostatic agents, 4) (semi-) professional athletes, 5) polydipsia, 6) pregnancy, and 7) hormonal (infertility) treatment. Three years after baseline assessment (T0), all participants were invited to undergo a second DEXA scan (T1).

Forty-three percent $(n=76)$ of the original sample at T0 was lost to follow-up at T1. Participants either met exclusion criteria at T1 ( $n=19$, most common exclusion criteria were use of corticosteroids and pregnancy) or they declined further participation for personal or practical reasons. We were unable to contact 14 of the original participants, 11 of whom were patients.

At T1, 30 patients with a psychotic disorder, 44 non-psychotic siblings and 27 controls remained. Twenty-three patients had a diagnosis of schizophrenia, 4 patients had a diagnosis of schizoaffective disorder and 3 patients had a diagnosis of psychotic disorder not otherwise specified. No controls or siblings transitioned to the patient group. Ten controls and 12 siblings had a current or past diagnosis 
of major depressive disorder (MDD), of whom four controls and two siblings transitioned from no diagnosis to MDD between T0 and T1.

The sample included 14 families. Ten families contributed one patient and one sibling and one family contributed one patient and two siblings. Two families contributed two siblings but no patients. In the control group, one family contributed two siblings. Nineteen independent patients, 28 independent siblings and 25 independent controls participated.

There were also participants at T1 who did not undergo a DEXA scan at T0. This additional group included 24 controls, 15 siblings and 12 patients. Thus, the total sample size at T1 was $n=152$.

\section{Measures}

Substance use

Substance use was assessed using the Composite International Diagnostic Interview (CIDI) (WHO, 1990). Cannabis use was assessed as reported frequency of use in the past year. Other drug use was assessed in the same way. Alcohol use was defined as the average number of weekly instances of consumption during the previous 12 months. Tobacco use was defined as the number of daily occasions of use.

\section{Physical activity and sunlight exposure}

Physical activity and sunlight exposure were expressed in total minutes per week at T1. To quantify physical activity, the total amount of time spent on commuting by foot or bicycle, physical activity at work or school, household chores, active hobbies and sports was summed. Sunlight exposure was calculated by multiplying the number of days per week a person went outside by the average number of minutes spent outside (during daylight) on those days.

\section{Use of antipsychotic medication}

Current AP use and AP use during the follow-up period were classified by AP type: prolactin (PRL) -raising, i.e. first generation APs, and risperidone, paliperidone and amisulpride; or PRL-sparing, i.e. second and third generation APs with the exception of risperidone, paliperidone and amisulpride. Previous AP use was assessed retrospectively by self-report. Best estimate cumulative AP exposure was determined by multiplying the number of days of AP use with the daily AP dose converted to haloperidol equivalents (in milligrams), and summing all periods of use. Exposure during the 3 year follow-up period and lifetime were calculated for all APs and separately for prolactin-raising APs.

\section{Use of contraceptive drugs (exogenous estrogen exposure)}

Exogenous estrogen exposure was determined by multiplying the daily dose expressed in micrograms with the total days of use. Exposure was determined for the three year interval between T0 and T1 as well as lifetime exposure up to T1. 
Age at menarche and dysmenorrhea

Age at menarche and the occurrence of menstrual irregularity were assessed in women.

\section{Familial osteoporosis}

The occurrence of familial osteoporosis was documented.

\section{Dual X-ray absorptiometry acquisition and processing}

Dual X-ray absorptiometry (DEXA) scans were acquired at Maastricht University Medical Centre with a Hologic Discovery A (Tromp Medical, Castricum, the Netherlands) (NHANES and Ethnic Reference Data) at both T0 and T1. The same procedures for DEXA acquisition were used at both measurements. There were no update in the machinery or software during the interval. DEXA scans of two anatomical areas were performed: the lumbar spine, vertebrae $L 2$ through $L 4$; and the proximal left femur, specifically the collum, trochanter major, intertrochanteric area and Ward's triangle. BMD was expressed in grams per square centimeter $\left(\mathrm{g} / \mathrm{cm}^{2}\right)$, Z-scores and T-scores. The Z-score compares an individual's BMD with the mean BMD of a comparable population (with respect to gender, age and ethnicity). The T-score compares an individual's BMD to peak bone mass (PBM). Peak bone mass is the highest BMD an individual is expected to acquire during life. The T-score is used to diagnose osteopenia and osteoporosis. The World Health Organization employs the following criteria: T-scores in osteopenia lie between -1.0 and -2.5; T-scores in osteoporosis are equal or less than -2.5.

At T1 we encountered an unanticipated complication. Pediatric reference values were applied to participants under the age of 20 at TO. These reference values were apparently more stringent than the references employed for adults. For instance, a female control was age 18 at T0 and the pediatric reference values were used. Though her total femoral BMD declined slightly over the three year follow-up ( $0.853 \mathrm{~g} / \mathrm{cm}^{2}$ at T0 and $0.845 \mathrm{~g} / \mathrm{cm}^{2}$ at T1), Z- and T-scores appeared to improve using adult reference values at age 21 ( $\mathrm{Z}$ : score -1.2 at T0 and -0.8 at T1; and T-score:

-1.3 at $\mathrm{T} 0$ and -0.8 at $\mathrm{T} 1)$.

The present longitudinal study included 4 controls, 6 siblings and 1 patient that had not reached age 20 at T0. Inclusion of these individuals would result in skewed lumbar and femoral delta Z- and T-scores, potentially cancelling out or creating differences between groups. Therefore, these 11 participants were excluded from the Z- and T-score analyses. For the total BMD analyses, we did include these individuals as the absolute BMD is not influenced by age-related reference values.

The study was approved by the standing ethics committee and all participants gave written informed consent in accordance with the committee's guidelines. 


\section{Statistical analyses}

Group differences in BMD measures at T1 $(n=152)$ and BMD change between T0 and T1 (total BMD lumbar spine and femur $n=101$, Z- and T-scores $n=90$ ) were analyzed separately for the lumbar spine and proximal femur. The change (delta, $\Delta$ ) in lumbar and femoral total BMD, Z-scores and T-scores was calculated as T1 minus T0. Multilevel random regression models were fitted (Goldstein, 1987) given hierarchical clustering occasioned by the fact that participants were clustered in families, compromising statistical independence of the observations. This was done using the XTREG command in STATA (STATA corp, version 13). BMD measures were the dependent variables in the analyses and group (entered as a linear and dummy variable (controls=0, siblings=1 and patients=2)) was the independent variable. Analyses were adjusted for the a priori hypothesized confounders: gender, age at T1, BMI at T1, exogenous estrogen exposure between T0 and T1, physical activity, sunlight exposure, tobacco and alcohol use, exposure to cannabis and the number of days between T0 and T1. Analyses were repeated with lifetime estrogen exposure replacing 3 year estrogen exposure as a covariate. Results were Simes corrected.

Despite the gender-specific findings in our original study (van der Leeuw et al., 2013a), we did not conduct analysis stratified by sex due to low numbers (only 7 men in the control group and 6 women in the patient group).

Planned sensitivity analyses were performed excluding individuals with a history of major depressive disorder (MDD) in the control and sibling groups because these individuals may be at risk of decreased BMD due to similar factors as in psychotic disorder, such as inactivity, diminished sunlight exposure and medication side-effects.

Main effect of the type of AP use (at T1 and during 3 year interval) on $\triangle$ BMD was examined by comparing PRL-raising AP to PRL-sparing and no AP (the latter two combined into one group) using multiple regression procedures; with sex, age and BMI as confounders. Accordingly, the main effect of cumulative exposure to PRL-raising AP (over the 3 year interval and lifetime) on $\triangle B M D$ was assessed. Sensitivity analyses were repeated with the further exclusion of patients who used a PRL-raising AP.

\section{RESULTS}

The descriptive characteristics apply to the longitudinal sample. The findings for the cross-sectional sample at T1 ( $n=152)$ and the longitudinal sample are presented separately.

\section{Descriptive characteristics}

There were more female participants in the control group and more males in the patient group. Controls were older than patients and siblings. Patients used more tobacco, cannabis and other drugs than controls and siblings. Siblings used more cannabis than controls. Among women, patients experienced more menstrual irregularities than siblings and controls (Table 1). 
Table 1. Demographics

\begin{tabular}{|c|c|c|c|}
\hline & Controls, $n=27$ & Siblings, $n=44$ & Patients, $\mathrm{n}=30$ \\
\hline Sex (n), male / female & $7 / 20$ & $26 / 18$ & $24 / 6$ \\
\hline Age at scan & $35.22 \pm 11.60$ & $32.32 \pm 7.97$ & $32.03 \pm 6.04$ \\
\hline Body Mass Index (BMI) (kg per m²) & $25.24 \pm 4.49$ & $24.02 \pm 3.94$ & $25.35 \pm 4.47$ \\
\hline Age at menarche (years) & $13.35 \pm 1.50$ & $12.67 \pm 1.24$ & $13.17 \pm 1.17$ \\
\hline Dysmenorrhea (n) & 9 & 6 & 5 \\
\hline 3 year exogenous estrogen exposure $(\mu \mathrm{g})$ & $5691 \pm 8057$ & $8995 \pm 10882$ & $9551 \pm 11072$ \\
\hline Lifetime exogenous estrogen exposure $(\mu \mathrm{g})$ & $34542 \pm 27201$ & $46674 \pm 48460$ & $25090 \pm 42206$ \\
\hline Smoking (cigarettes per day) & $1.52 \pm 3.61$ & $2.61 \pm 6.39$ & $12.43 \pm 10.71$ \\
\hline Alcohol consumption (units per week) & $7.23 \pm 13.74$ & $6.75 \pm 7.16$ & $6.17 \pm 9.22$ \\
\hline Cannabis use (number of times past year) & $0.30 \pm 1.03$ & $8.75 \pm 54.88$ & $66.83 \pm 138.69$ \\
\hline Other drug use (number of times past year) & 0 & $0.05 \pm 0.30$ & $3.47 \pm 10.01$ \\
\hline Activity (minutes per week) & $2242 \pm 1299$ & $1601 \pm 1146$ & $1471 \pm 1173$ \\
\hline Sunlight exposure (minutes per week) & $945 \pm 565$ & $1084 \pm 820$ & $955 \pm 868$ \\
\hline Current AP use (n, patients) & & & 26 \\
\hline PRL-raising / PRL-sparing AP (n) & & & $12 / 14$ \\
\hline Current AP dose (hal. eq.) & & & $4.49 \pm 3.38$ \\
\hline 3 year PRL-raising AP exposure (hal. eq.) & & & $1711 \pm 2609$ \\
\hline Lifetime PRL-raising AP exposure (hal. eq.) & & & $5998 \pm 7117$ \\
\hline 3 year all AP exposure (hal. eq.) & & & $4899 \pm 3933$ \\
\hline Lifetime all AP exposure (hal. eq.) & & & $12375 \pm 10069$ \\
\hline
\end{tabular}

Means \pm SDs reported.

AP: antipsychotic medication; PRL: prolactin; hal. eq.: haloperidol equivalents in milligrams.

Table 2. Cross-sectional associations between group and BMD measures at T1

\begin{tabular}{lllll}
\hline & Linear trend & S vs. C & P vs. C & P vs. S \\
\hline Lumbar spine & & & & \\
Total BMD & $0.029(0.081)$ & $0.042(0.069)$ & $0.051(0.118)$ & $0.009(0.757)$ \\
Z-score & $0.199(0.188)$ & $0.270(0.199)$ & $0.359(0.229)$ & $0.089(0.746)$ \\
T-score & $0.273(0.072)$ & $0.394(0.061)$ & $0.481(0.108)$ & $0.087(0.752)$ \\
Femur & & & & \\
Total BMD & $0.009(0.535)$ & $0.005(0.817)$ & $0.021(0.481)$ & $0.016(0.558)$ \\
Z-score & $0.044(0.688)$ & $0.014(0.929)$ & $0.104(0.631)$ & $0.091(0.651)$ \\
T-score & $0.044(0.686)$ & $0.025(0.870)$ & $0.098(0.650)$ & $0.073(0.714)$ \\
\hline
\end{tabular}

B's (and p-values) reported. B represents the regression coefficient of the multilevel regression analyses.

S vs. C: siblings vs. controls; P vs. C: patients vs. controls; P vs. S: patients vs. siblings. 
Out of 30 patients, 26 currently used AP medication, of whom 14 were prescribed a PRL-sparing agent and 12 were prescribed PRL-raising agents (1 patient used haloperidol, 8 risperidone, 1 paliperidone and 1 amisulpride; one patient used a combination of risperidone and aripiprazole). Of the 4 patients who were AP-free at T1, two were AP-free during the entire 3 year interval, and 2 used a PRL-sparing AP (1 aripiprazole, 1 olanzapine) between T0 and T1. The 14 patients with a PRL-sparing AP at T1 did not use a PRL-raising AP at any time during the 3 year interval. However, with the exception of 4 individuals, all patients did use a PRL-raising AP at some point during their lives. Cumulative AP exposure is listed in Table 1. Several patients used other classes of psychotropic medication: eight used antidepressants (of whom 7 a selective serotonin reuptake inhibitor (SSRI) or serotonin and noradrenalin reuptake inhibitor (SNRI), and 1 trazodone), three used benzodiazepines and there was one instance of stimulant (methylphenidate) use. One sibling used mirtazapine and one control used paroxetine, both had a history of MDD. One sibling had briefly used a benzodiazepine due to anxiety (no diagnosis was made), and one sibling and one control had briefly used diazepam due to back pain.

\section{Associations between group and BMD in the lumbar spine and femur}

Cross-sectional analyses

There were no statistical associations between group (linear trend and dummy variables) and BMD measures at T1 (Table 2). Although not significantly so, all B's were positive, including the patientcontrol and patient-siblings comparisons.

\section{Longitudinal analyses}

Mean BMD measures at T0 and T1 are depicted in Figure 1. Only patients showed incremental mean BMD change, specific to the lumbar spine. The longitudinal associations between group and BMD change are stated in Table 3. Depending on the direction of mean BMD change, a positive association in the group comparisons signifies either an increase or smaller decrease in BMD measures. There was a significant positive association between group (linear trend) and the $\Delta$ Z-score of the femur $(B=0.111,95 \%$ confidence interval $(C l) 0.008$ to $0.215, p=0.035)$. The association for the $\Delta$ total femoral $\mathrm{BMD}$ and $\triangle \mathrm{T}$-score were positive at trend-level significance (Table 3).

For the femur, there were positive associations between group and $\triangle B M D$ measures in the patientcontrol comparison (total BMD: $B=0.026,95 \% \mathrm{Cl} 0.002$ to $0.050, p=0.037$; Z-score: $B=0.224,95 \% \mathrm{Cl}$ 0.035 to $0.412, p=0.020$; and T-score: $B=0.193,95 \% \mathrm{Cl} 0.003$ to $0.382, p=0.046$ ). For the lumbar spine, there were no significant findings in any of the group comparisons (Table 3 ).

The use of lifetime estrogen exposure (instead of 3 year estrogen exposure) as a covariate in the analyses led to additional findings. The association between group (linear trend) and $\triangle$ femoral BMD measures became more apparent (total BMD: $B=0.015,95 \% \mathrm{Cl} 0.001$ to $0.029, p=0.032$; Z-score: $B=0.139,95 \% \mathrm{Cl} 0.031$ to $0.246, p=0.012$; and T-score: $B=0.123,95 \% \mathrm{Cl} 0.016$ to $0.231, p=0.025)$. The significant positive associations in $\triangle$ femoral $B M D$ values in patients compared to controls was upheld (total BMD:B=0.032, 95\% Cl 0.007 to 0.058, $p=0.013$; Z-score: $B=0.280,95 \% \mathrm{Cl} 0.085$ to $0.475, p=0.005$; 
and T-score: $\mathrm{B}=0.248,95 \% \mathrm{Cl} 0.053$ to $0.443, \mathrm{p}=0.013)$, and $\triangle$ total femoral $\mathrm{BMD}$ and $\mathrm{Z}$-score in patients compared to siblings became statistically significant (total BMD: $B=0.024,95 \% \mathrm{Cl} 0.002$ to 0.045 , $\mathrm{p}=0.033$; Z-score: $\mathrm{B}=0.170,95 \% \mathrm{Cl} 0.004$ to $0.337, \mathrm{p}=0.045$ ).

For the lumbar spine, there were no significant findings in any of the group comparisons. The significant findings described above were not upheld after Simes correction.

Table 3. Longitudinal associations between group and $\triangle B M D$ measures

\begin{tabular}{lllll}
\hline & Linear trend & S vs. C & P vs. C & P vs. S \\
\hline Lumbar spine & & & & \\
Total BMD & $-0.002(0.813)$ & $-0.002(0.802)$ & $-0.003(0.812)$ & $-0.001(0.954)$ \\
Z-score & $0.003(0.963)$ & $0.013(0.910)$ & $0.006(0.965)$ & $-0.006(0.956)$ \\
T-score & $-0.002(0.979)$ & $0.020(0.856)$ & $-0.006(0.966)$ & $-0.026(0.824)$ \\
Femur & & & & \\
Total BMD & $0.012(0.063)$ & $0.008(0.381)$ & $0.026(0.037)^{*}$ & $0.018(0.092)$ \\
Z-score & $0.111(0.035)^{*}$ & $0.100(0.195)$ & $0.224(0.020)^{*}$ & $0.123(0.130)$ \\
T-score & $0.097(0.068)$ & $0.101(0.195)$ & $0.193(0.046)^{*}$ & $0.092(0.262)$ \\
\hline
\end{tabular}

B's (and p-values) reported. B represents the regression coefficient of the multilevel regression analyses.

*p-value $<0.05$.

\section{Associations between prolactin-raising antipsychotic medication and BMD change}

Delta BMD measures were not significantly different in patients who used a PRL-raising AP compared to those who used a PRL-sparing AP or were AP-free (both at the time of DEXA acquisition and during the entire three year follow-up period). Neither the 3 year nor the lifetime cumulative exposure to PRL-raising APs was associated with $\triangle \mathrm{BMD}$ (results not shown). Exposure to all AP during the 3 year interval had very small though statistically significant effects on $\triangle$ femoral BMD measures (total BMD: $\mathrm{B}=4.93 \cdot 10^{-6}, 95 \% \mathrm{Cl} 1.07 \cdot 10^{-6}$ to $8.79 \cdot 10^{-6}, \mathrm{p}=0.014$; $\mathrm{Z}$-score: $\mathrm{B}=2.64 \cdot 10^{-5}, 95 \% \mathrm{Cl} 1.79 \cdot 10^{-8}$ to $5.29 \cdot 10$ 5, $p=0.050$; T-score $B=3.16 \cdot 10^{-5}, 95 \% \mathrm{Cl} 4.63 \cdot 10^{-6}$ to $5.86 \cdot 10^{-5}, p=0.024$ ), that did not survive Simes correction. Lifetime exposure to all AP did not influence BMD change.

\section{Exclusion of affective disorder and prolactin-raising antipsychotic medication}

Cross-sectional analyses

Exclusion of siblings and controls with a history of MDD from the analysis did not result in significant findings at T1. Exclusion of patients who used a PRL-raising AP resulted in an isolated finding of a significant positive association in the patient-sibling comparison for the total femoral $B M D(B=0.076$, 95\% Cl: 0.004 to $0.147, p=0.038$ ), which did not survive Simes correction. 


\section{Longitudinal analyses}

Exclusion of siblings and controls with a history of MDD from the analysis yielded similar results for the lumbar spine (no $\triangle B M D$ differences between groups). As in the main analysis, there was a significant positive association between group (linear trend) and the $\Delta \mathrm{Z}$-score of the femur $(B=0.131,95 \% \mathrm{Cl}$ 0.011 to $0.251, p=0.033$ ), while the association for total femoral BMD and the T-score at trend-level significance (total $B M D: B=0.015,95 \% C l-0.001$ to $0.031, p=0.059$; and T score: $B=0.116,95 \% \mathrm{Cl}-0.004$ to $0.235, p=0.058$ ). The positive associations between group and $\triangle$ femoral BMD measures in the patient-control comparison remained (total BMD: $\mathrm{B}=0.031,95 \% \mathrm{Cl} 0.003$ to $0.059, \mathrm{p}=0.030$; Z-score: $B=0.260,95 \% \mathrm{Cl} 0.050$ to $0.471, p=0.015$; and T-score: $B=0.230,95 \% \mathrm{Cl} 0.019$ to $0.440, p=0.033)$. In addition, the associations in the patient-sibling comparison became significant (total $B M D: B=0.027$, 95\% Cl 0.003 to 0.051 , $p=0.027$; Z-score: $B=0.210,95 \% \mathrm{Cl} 0.029$ to $0.390, p=0.023$; and T-score: $B=0.179$, $95 \% \mathrm{Cl} 0.001$ to $0.360, \mathrm{p}=0.052$ ). After further exclusion of patients who used a PRL-raising AP from the analyses only the patient-control comparison in femoral BMD changes remained partially significant (total BMD: $B=0.032,95 \% \mathrm{Cl}-0.001$ to $0.066, p=0.058$; Z-score: $B=0.269,95 \% \mathrm{Cl} 0.012$ to $0.526, p=0.040$; and T-score: $B=0.254,95 \% \mathrm{Cl} 0.001$ to $0.508, p=0.049$ ).

All findings described above were not upheld after Simes correction.

Figure 1. Mean BMD measures at $\mathrm{T} 0$ and $\mathrm{T} 1$ in the longitudinal sample

\section{Lumbar spine}

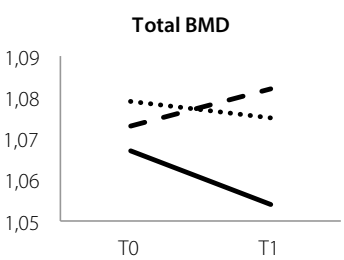

Femur

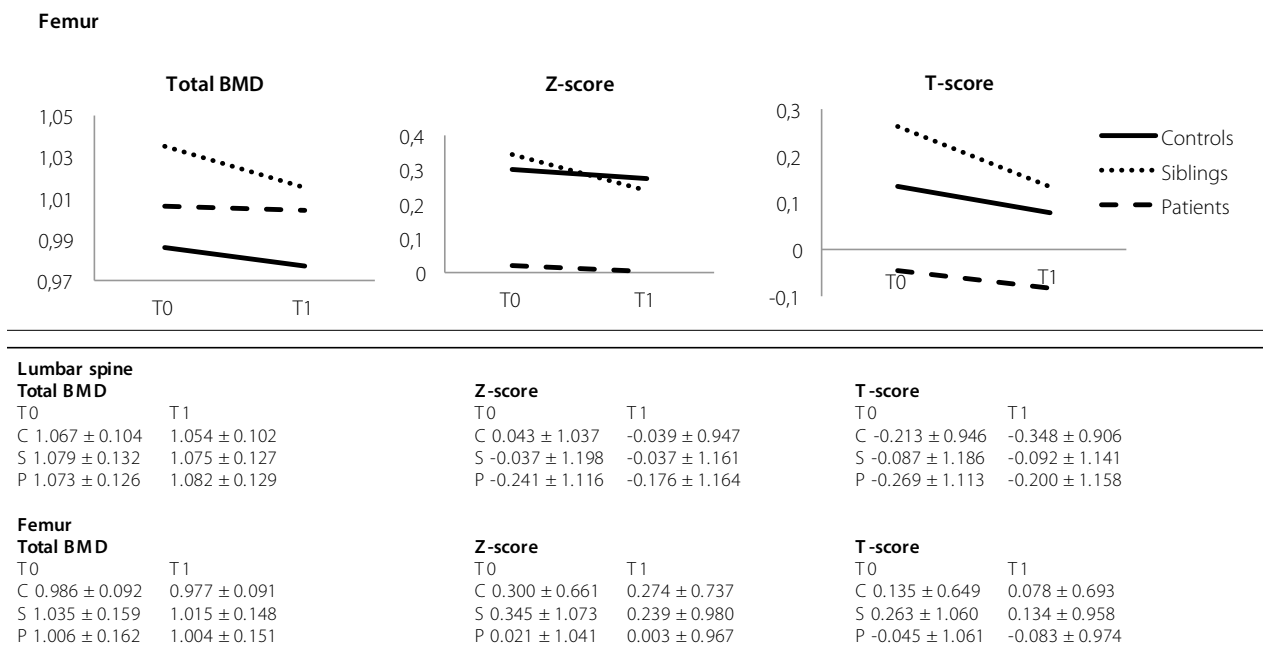

Z-score

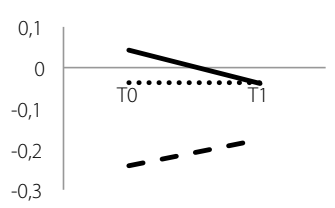

T-score

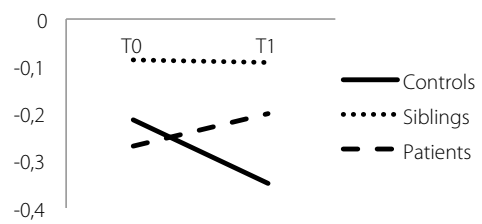




\section{DISCUSSION}

Contrary to our hypothesis, BMD did not decrease disproportionately in patients compared to siblings and controls. The current or past use of a prolactin-raising AP was not found to be negatively associated with $\triangle B M D$ measures.

\section{Findings}

The finding of the original study (van der Leeuw et al., 2013a) at T0, namely reduced femoral BMD in female patients (as well as a trend in the whole patient group), is consistent with the conclusions of a review (Kishimoto et al., 2012) and meta-analysis (Stubbs et al., 2014), i.e. lower BMD is prevalent in schizophrenia. Replication of the main baseline findings (reduced femoral BMD in female patients) was not possible in the follow-up sample due to considerable sample size reduction and related power loss, preventing the realization of analyses stratified by sex. Despite evident group differences in risk factors such as smoking, no statistical differences between groups were found in the cross-sectional analysis at T1. Similar to the results at T0 (Van der Leeuw et al., 2013a), siblings were not statistically different from controls at $\mathrm{T} 1$, confirming that BMD does not appear to be an endophenotype of psychotic disorder.

The lines in the graphs in Figure 1 displaying mean femoral BMD measures at T0 and T1 seem to converge slightly, possibly suggesting a diminished difference at $\mathrm{T} 1$. Indeed, our findings do not indicate excessive loss of BMD in patients. Instead, femoral BMD measures appeared to decrease less in the patient versus control comparison (reflected by a significant positive B in the regression analyses), although these findings were not upheld after Simes correction. Controls, with a mean age of 35 and 3 years senior to siblings and patients, showed a drop in all mean lumbar BMD measures and total femoral BMD. This is compatible with normal bone physiology as peak bone mass is retained until around age 30 and then begins to decline. As age is the only moderator of bone mass loss that survives Bonferroni correction in meta-analysis (Stubbs et al., 2014), our patients were possibly too young at T1 to show disproportionate BMD loss compared to (slightly older) controls. Matching for gender and age would have enabled a better comparison.

Notably, patients currently using PRL-raising AP did not show accelerated BMD loss compared to patients taking PRL-sparing AP or no AP. Nor did cumulative three year or lifetime exposure to PRLraising AP have a main effect on BMD change. Exposure to all AP in the 3 year interval appeared to have a very small positive effect, of which the clinical relevance is questionable.

The interpretation of our findings concerning AP are further complicated by the fact that AP data was largely collected retrospectively by self-report. This is prone to bias. Ideally, the participant supplied an overview of medication history from their doctor or pharmacy. It was standard practice to request this of the participant, though participants did not routinely comply.

The meta-analysis by Stubbs reports that neither PRL-raising AP use nor confirmed hyperprolactinemia contributed to the higher prevalence of low bone mass in schizophrenia; however, they conclude that 
heterogeneity between studies may underlie the absence of association (Stubbs et al., 2014).

More optimistically, our findings may be reassuring for patients who take prolactin-raising AP in the short term. In the long run, it deserves recommendation to remain cautious of sequelae of hyperprolactinemia such as hypogonadism and BMD loss, and to assess side-effects of prolactinraising AP in each individual patient.

\section{Longitudinal studies}

Longitudinal studies investigating BMD in psychotic disorder are scarce. Four other studies have been carried out to date. Recently, in a larger study (163 patients and 90 controls), Wang and colleagues found that hyperprolactinemia resulted in decreased total lumbar BMD in a group of male and female patients with schizophrenia (mean age of 36) who had been treated with PRL-raising APs for 12 months (Wang et al., 2014). An observational cohort study (Takahashi et al., 2013) examined longitudinal BMD changes in patients, comparing patients taking PRL-raising AP ( $n=141)$ and PRLsparing AP ( $n=23$ ) at first DEXA measurement. The mean follow-up and main finding is comparable to the present study. There was no significant BMD difference between patients taking PRL-raising or PRL-sparing AP at each measurement, over a mean follow-up period of 3.4 years. However, a significant AP type by time interaction was found, possibly indicating a negative effect of prolactin-raising AP. Two studies examined AP effects on BMD specifically in women between the ages of 22 and 53 years (mean 36 years) (Abraham et al., 2003) and at premenopausal age (not specified in years) (Meaney and O'Keane, 2007). Over a period of 12 months, Abraham et al. found that hyperprolactinemia accelerated bone metabolism, but this did not result in bone mass loss (Abraham et al., 2003). The latter finding (absence of bone mass loss) is consistent with the current findings in patients (although both sexes were included in our study), in whom we did not find significant changes in BMD despite the common use of PRL-raising AP from a lifetime perspective. Meaney and O'Keane (Meaney and O'Keane, 2007) followed premenopausal female patients with schizophrenia over the course of one year and compared lumbar and femoral BMD changes between those taking prolactin-sparing AP and those taking prolactin-raising AP. In that study, the efficacy of interventions (such as weightbearing exercise, nutritional supplements and sex hormone replacement therapy) to ameliorate BMD was examined. Without intervention, women on a PRL-raising AP showed a decrease in lumbar BMD compared to women taking a PRL-sparing AP. With intervention, patients using PRL-sparing AP $(n=4)$ showed a significant increase in lumbar BMD, while patients using a PRL-raising AP $(n=12)$ also showed an increase, though not significantly so (Meaney and O'Keane, 2007). No differences in femoral BMD were found, between the AP or intervention groups. Although the incremental change was specific to total femoral BMD in our study, Meaney and O'Keane's findings in the lumbar spine lend some support to our findings as they show a beneficial evolution of BMD change in a small group of patients using PRL-sparing AP. The majority of our patients used PRL-sparing AP or no AP. An alternative explanation for the absence of progressive BMD loss in the patient group of the current study is that therapeutic interventions may have occurred to improve BMD over the course of the 3 
year follow-up in participants with low BMD. When the DEXA scan at T0 was indicative of osteopenia or osteoporosis, the participant's general practitioner was alerted and treatment may have been started. As the study design was naturalistic, these interventions were not monitored between T0 and $\mathrm{T} 1$ and did not constitute an exclusion criterion at T1.

\section{Methodological considerations}

Our study makes a new contribution to the existing literature, despite some limitations. At T0 study size was moderate. Due to a loss to follow-up of $43 \%$, sample size at $\mathrm{T} 1$ was reduced quite drastically. Thus, the longitudinal analyses may have been underpowered and the possibility of false-negative outcomes cannot be excluded. As the main finding of reduced femoral BMD in the original study (T0) was specific to the female patient sample, we wished to reexamine sex differences at T1. Female patient sample size was already marginal at T0 $(n=16)$, unfortunately this number declined further at T1 $(n=6)$ and stratified analyses per sex were not deemed feasible. Larger longitudinal studies are warranted.

The present study had an adequate follow-up duration, comparable to only one other study (Takahashi et al., 2013) that had a similar mean follow-up with a maximum up to five years. Abraham et al. argued that their follow-up of one year may have been insufficient for changes in bone metabolism to result in actual loss of BMD (Abraham et al., 2003). The three year follow-up in this study did not indicate disproportionate loss of BMD, though markers of bone metabolism were not available to examine bone turnover rates and serum prolactin measurements were lacking. Furthermore, sex hormone and prolactin levels were not assessed. Interpretation of estrogen levels is complicated as its measurement is a momentary assessment of naturally fluctuating concentrations. Given our primary interest in cumulative estrogen exposure, BMD was considered the best outcome measure. Nevertheless, although estrogen and prolactin levels do not represent lifetime exposure, they may have contributed to the understanding of our findings.

Serum vitamin D concentration would have been another useful laboratory measure. It would certainly have been superior to the variable sunlight exposure (in minutes per week spent outside during daylight). Physical activity was also expressed in minutes per week, calculated by a self-report questionnaire conceived for this study. The use of a standardized instrument to measure physical activity would have been desirable. Soundy and colleagues delineate recommendations in their review (Soundy et al., 2014) for future practice. Self-report questionnaires are subject to recall bias, even more so in studies including participants with cognitive symptoms due to schizophrenia.

The inclusion of siblings and controls with MDD in the study sample is another issue worth addressing. This issue was addressed by correcting for (shared) risk factors between psychotic disorder and MDD in the primary analysis. Furthermore, controls and siblings with MDD were excluded in sensitivity analyses (again to account for these risk factors as well as the sparse use of medication among siblings and controls with MDD as described under 3.1 Descriptive characteristics). 
Due to the issue of discrepant pediatric and adult reference values (described under section 2.3), total BMD change (larger sample, $\mathrm{n}=101$ ) should serve as a benchmark with which Z- and T-score changes (in smaller sample of 90) should correspond. This was the case for the majority of our findings. Therefore, we feel that we adequately addressed issue of discrepant reference values and our findings were not greatly influenced by this caveat.

A major distinction of the present study is that it is unique in its focus on a possible etiological mechanism, i.e. (persisting) primary low estrogen levels. However, designing a study to elucidate a primary etiological effect for estrogen in psychotic disorder remains challenging. Although BMD was used as a marker of cerebral estrogen exposure, we should bear in mind that different tissue sites have different estrogenic receptors and effects. Furthermore, BMD is influenced by many (non-) hormonal factors. It is, at best, a proxy marker of estrogen-mediated neuroprotection.

\section{Conclusions}

In this small longitudinal study, there was no evidence of ongoing estrogen deficiency in psychotic disorder as there was no excessive BMD loss over a 3-year period in patients using AP. No proof of effects of disease-related factors or PRL-raising AP on BMD was found.

In line with previous cross-sectional work, BMD did not appear to be an endophenotypic marker of psychotic disorder. 


\section{REFERENCES}

Abraham, G., Paing, W.W., Kaminski, J., Joseph, A., Kohegyi, E., Josiassen, R.C., 2003. Effects of elevated serum prolactin on bone mineral density and bone metabolism in female patients with schizophrenia: a prospective study. Am J Psychiatry 160, 1618-1620.

Andreasen, N.C., Flaum, M., Arndt, S., 1992. The Comprehensive Assessment of Symptoms and History (CASH). An instrument for assessing diagnosis and psychopathology. Arch Gen Psychiatry 49, 615-623.

APA, 2000. Diagnostic and statistical manual of mental disorders, 4th edition. American Psychiatric Association, Washington, D.C.

Bilici, M., Cakirbay, H., Guler, M., Tosun, M., Ulgen, M., Tan, U., 2002. Classical and atypical neuroleptics, and bone mineral density, in patients with schizophrenia. Int J Neurosci 112, 817-828.

Boerma, M.A., van der Stel, J.C., van Amelsvoort, T., Linszen, D.H., de Haan, L., 2010. [Women, schizophrenia and oestrogen; neurobiological hypotheses and hormonetherapy studies]. Tijdschrift voor psychiatrie 52, 235-244.

Clemons, M., Goss, P., 2001. Estrogen and the risk of breast cancer. N Engl J Med 344, 276-285.

Crews, M.P., Howes, O.D., 2012. Is antipsychotic treatment linked to low bone mineral density and osteoporosis? A review of the evidence and the clinical implications. Human psychopharmacology 27, 15-23.

Goldstein, H., 1987. Multilevel Models in Educational and Social Research. Griffin, London.

Haddad, P.M., Wieck, A., 2004. Antipsychotic-induced hyperprolactinaemia: mechanisms, clinical features and management. Drugs 64, 2291-2314.

Halbreich, U., 2007. Osteoporosis, schizophrenia and antipsychotics: the need for a comprehensive multifactorial evaluation. CNS Drugs 21, 641-657.

Howard, L., Kirkwood, G., Leese, M., 2007. Risk of hip fracture in patients with a history of schizophrenia. Br J Psychiatry 190, 129-134.

Kishimoto, T., De Hert, M., Carlson, H.E., Manu, P., Correll, C.U., 2012. Osteoporosis and fracture risk in people with schizophrenia. Curr Opin Psychiatry 25, 415-429.

Korver, N., Quee, P.J., Boos, H.B., Simons, C.J., de Haan, L., 2012. Genetic Risk and Outcome of Psychosis (GROUP), a multi-site longitudinal cohort study focused on gene-environment interaction: objectives, sample characteristics, recruitment and assessment methods. International journal of methods in psychiatric research 21, 205-221.

Maric, N., Popovic, V., Jasovic-Gasic, M., Pilipovic, N., van Os, J., 2005. Cumulative exposure to estrogen and psychosis: a peak bone mass, case-control study in first-episode psychosis. Schizophrenia research 73, 351-355.

Markham, J.A., 2012. Sex steroids and schizophrenia. Rev Endocr Metab Disord 13, 187-207.

Meaney, A.M., O'Keane, V., 2007. Bone mineral density changes over a year in young females with schizophrenia: relationship to medication and endocrine variables. Schizophrenia research 93, 136-143.

Meaney, A.M., Smith, S., Howes, O.D., O'Brien, M., Murray, R.M., O'Keane, V., 2004. Effects of long-term prolactinraising antipsychotic medication on bone mineral density in patients with schizophrenia. Br J Psychiatry 184, 503-508.

Partti, K., Heliovaara, M., Impivaara, O., Perala, J., Saarni, S.I., Lonnqvist, J., Suvisaari, J.M., 2010. Skeletal status in psychotic disorders: a population-based study. Psychosom Med 72, 933-940.

Soundy, A., Roskell, C., Stubbs, B., Vancampfort, D., 2014. Selection, use and psychometric properties of physical activity measures to assess individuals with severe mental illness: a narrative synthesis. Archives of psychiatric nursing 28, 135-151.

Stubbs, B., De Hert, M., Sepehry, A.A., Correll, C.U., Mitchell, A.J., Soundy, A., Detraux, J., Vancampfort, D., 2014. A meta-analysis of prevalence estimates and moderators of low bone mass in people with schizophrenia. Acta psychiatrica Scandinavica 130, 470-486. 
Takahashi, T., Uchida, H., John, M., Hirano, J., Watanabe, K., Mimura, M., Correll, C.U., Kishimoto, T., 2013. The impact of prolactin-raising antipsychotics on bone mineral density in patients with schizophrenia: findings from a longitudinal observational cohort. Schizophrenia research 147, 383-386.

van der Leeuw, C., Habets, P., Domen, P., van Kroonenburgh, M., van Os, J., Marcelis, M., 2013a. Bone mineral density as a marker of cumulative endogenous estrogen exposure: Relationship to background genetic risk of psychotic disorder. Schizophrenia research 143, 25-31.

van der Leeuw, C., Habets, P., Gronenschild, E., Domen, P., Michielse, S., van Kroonenburgh, M., van Os, J., Marcelis, M., 2013b. Testing the estrogen hypothesis of schizophrenia: associations between cumulative estrogen exposure and cerebral structural measures. Schizophrenia research 150, 114-120.

Wang, M., Hou, R., Jian, J., Mi, G., Qiu, H., Cao, B., Tang, M., 2014. Effects of antipsychotics on bone mineral density and prolactin levels in patients with schizophrenia: a 12-month prospective study. Human psychopharmacology 29, 183-189.

WHO, 1990. Composite International Diagnostic Interview (CIDI). World Health Organization, Geneva. 
a Department of Psychiatry and Psychology, School for Mental Health and Neuroscience, European Graduate School of Neuroscience, Maastricht University Medical Centre, Maastricht, the Netherlands

b Departments of Neurology and Laboratory Medicine, Radboud University Medical Centre, Nijmegen, the Netherlands

c Donders Institute for Brain, Cognition and Behaviour, Nijmegen, the Netherlands

d Central Diagnostic Laboratory, Maastricht University Medical Centre, Maastricht, the Netherlands

e Deparment of Psychiatry, Academic Medical Centre, Amsterdam, the Netherland

$f$ King's College London, King's Health Partners, Department of Psychosis Studies, Institute of Psychiatry, London, UK

9 Departments of Psychiatry and Neuroscience, Erasmus Medical Centre, Rotterdam, the Netherlands

h Department "Nieuwe Kennis", Delta Centre for Mental Health Care, Rotterdam, the Netherlands 


\section{Five}

Replicated evidence of absence of association between serum S100B and (risk of) psychotic disorder

C. van der Leeuwa ${ }^{M D}$

M. Marcelis ${ }^{\mathrm{a}} \mathrm{MD}$ PhD

S. Peeters ${ }^{\mathrm{a}} \mathrm{MSC}$

M. Verbeek, ${ }^{b, c}$ PhD MSC

P. Menheere ${ }^{d}$ PhD

L. de Haane MD PhD

J. van Os, ${ }^{\mathrm{a}, \mathrm{f}} \mathrm{MD} \mathrm{PhD}$

N. van Bevereng,h MD PhD; for G.R.O.U.P. 


\section{ABSTRACT}

Background S100B is a potential marker of neurological and psychiatric illness. In schizophrenia, increased S100B levels, as well as associations with acute positive and persisting negative symptoms, have been reported. It remains unclear whether S100B elevation, which possibly reflects glial dysfunction, is the consequence of disease or compensatory processes, or whether it is an indicator of familial risk.

Methods Serum samples were acquired from two large independent family samples ( $\mathrm{n}=348$ and $n=254$ ) in the Netherlands comprising patients with psychotic disorder ( $n=140$ and $n=82$ ), nonpsychotic siblings of patients with psychotic disorder $(n=125$ and $n=94)$ and controls $(n=83$ and $\mathrm{n}=78$ ). S100B was analyzed with a Liaison automated chemiluminescence system. Associations between familial risk of psychotic disorder and S100B were examined.

Results Results showed that S100B levels in patients (P) and siblings (S) were not significantly different from controls (C) (dataset 1: P vs. $C: B=0.004,95 \% C l-0.005$ to $0.013, p=0.351 ; S$ vs. $C: B=0.000,95 \%$ $\mathrm{Cl}-0.009$ to $0.008, p=0.926$; and dataset 2: $P$ vs. $C: B=0.008,95 \% \mathrm{Cl}-0.011$ to $0.028, p=0.410 ; \mathrm{S}$ vs. $\mathrm{C}$ : $\mathrm{B}=0.002,95 \% \mathrm{Cl}-0.016$ to $0.021, \mathrm{p}=0.797)$. In patients, negative symptoms were positively associated with $\mathrm{S100B}(\mathrm{B}=0.001,95 \% \mathrm{Cl} 0.000$ to $0.002, \mathrm{p}=0.005)$ in one of the datasets, however with failure of replication in the other. There was no significant association between $\mathrm{S100B}$ and positive symptoms or present use or type of antipsychotic medication.

Conclusions S100B is neither an intermediate phenotype, nor a trait marker for psychotic illness. 


\section{INTRODUCTION}

The search for biological markers and endophenotypes is ongoing in the field of psychiatry. The term biomarker refers to a characteristic that can be objectively measured and evaluated as an indicator of normal and pathogenic biological processes (van Beveren and Hoogendijk, 2011). Their identification may offer insight into etiological mechanisms of disease and offer new treatment perspectives. In clinical practice, valid biomarkers assist in the diagnostic process and enable monitoring of disease course and treatment response (Pillai and Buckley, 2012; van Beveren and Hoogendijk, 2011).

$\mathrm{S} 100 \mathrm{~B}$ is a protein that has been described as a potential biomarker of neurological and psychiatric disease. It has been coined "the CRP (C-reactive protein) of the brain" (Sen and Belli, 2007), and is an indicator of central nervous system (CNS) pathology from birth until elderly age (Michetti et al., 2012). $\mathrm{S} 100 \mathrm{~B}$ is expressed in and secreted by glial cells, with dual effects: at physiological intracellular levels and nanomolar extracellular concentrations it is neurotrophic (Donato et al., 2013; Donato et al., 2009); at micromolar levels it becomes neurotoxic (Donato et al., 2013; Donato et al., 2009; Sen and Belli, 2007; van Beveren et al., 2006). Elevated serum S100B in schizophrenia has been fairly consistently reported since the first publication more than a decade ago (Wiesmann et al., 1999), and has been confirmed by a meta-analysis (Schroeter et al., 2009). This meta-analysis, however, was not based on systematic review and did not report on apparently high levels of heterogeneity. Also, studies included were typically very small and no analysis of possible publication bias was provided. A few studies reported absence of association or inconclusive findings (Gattaz et al., 2000; Schroeter et al., 2003; Steiner et al., 2006).

Initially, S100B elevation was ascribed to glial dysfunction or astrocytic activation (Rothermundt et al., 2009; Rothermundt et al., 2004a). It is not understood whether it results from passive release secondary to neuronal damage, or active secretion due to glial activation as a primary pathophysiological or compensatory mechanism. Indirect evidence points to active secretion illustrated by reports of isolated elevation of S100B levels, unaccompanied by established markers of neuronal damage (Schroeter et al., 2009; Steiner et al., 2006). In addition, increased S100B levels have been associated with increased metabolism in glial cells measured by magnetic resonance spectroscopy (Rothermundt et al., 2007). Clinically, elevated S100B may be associated with acute psychosis and persistent negative symptoms (Ling et al., 2007; Rothermundt et al., 2001; Rothermundt et al., 2004b; Schroeter et al., 2003). Histological findings indicate that glial activation may result in increased intracellular S100B in paranoid schizophrenia (positive symptoms), whereas white matter damage or dysfunction in residual schizophrenia (negative symptoms) may lead to extracellular release (Steiner et al., 2008).

More recently, S100B in schizophrenia has been related to glucose metabolism and insulin resistance (Steiner et al., 2010a). Altered glucose metabolism in schizophrenia is frequently associated with the use of second-generation antipsychotics, but changes in insulin resistance and insulin-related peptides have also been observed in antipsychotic-naïve patients (Guest et al., 2010; van Nimwegen et al., 2008), suggesting an etiologic role for hyperinsulinemia. The two mechanisms may not be mutually 
exclusive. Abnormal glucose metabolism (i.e. diabetes) leads to small vessel disease, which causes white matter lesions. Accordingly, glucose abnormalities may cause secondary glial dysfunction, with or without primary altered glial function.

Notably, both white matter irregularities (Camchong et al., 2009; Clark et al., 2011; Hao et al., 2009; Knochel et al., 2012) and glucose abnormalities (Fernandez-Egea et al., 2008a; Fernandez-Egea et al., 2008b; Mukherjee et al., 1989) have been reported in unaffected first-degree relatives of patients with schizophrenia. However, it is not known whether S100B changes exist in this group. Two Chinese studies have investigated S100B gene polymorphisms as possible indicators of susceptibility to schizophrenia. The first study (Liu et al., 2005) found that the V3-V4 (G-C) haplotype of the S100B gene was more prevalent in patients than controls, whereas the second study (Zhai et al., 2012) reported an absence of significant differences in genotypes or allele frequencies of S100B gene polymorphisms between patients and controls.

In summary, it remains unclear whether S100B elevation is associated with schizophrenia, and if so, whether the association reflects the consequence of acute or chronic disease processes, compensatory mechanisms or genetic risk for schizophrenia. We set out to test whether S100B levels are associated with (familial risk of) psychotic disorder, in two large and independent samples. We hypothesized that S100B levels would be elevated in both siblings and patients, suggesting that S100B elevation may be considered an intermediate phenotype.

\section{METHODS AND MATERIALS}

\section{Participants}

Data was collected in the context of an ongoing longitudinal multicentre study in the Netherlands (Habets et al., 2011; Korver et al., 2012). The present total study population comprised cross-sectional data from two large independent samples derived from: i) Amsterdam and Rotterdam, including surrounding areas, and ii) Maastricht and surrounding areas (extending into nearby Belgium) (hereafter referred to as the Amsterdam and Maastricht samples respectively). Patients with a minimum age of 16 years with a diagnosis of non-affective psychotic disorder were included. Patients were recruited through the mental health services where they were treated, either as in or outpatients. Diagnosis was based on DSM-IV criteria (Association, 2000), assessed with the Comprehensive Assessment of Symptoms and History (CASH) interview (Andreasen et al., 1992). Siblings were sampled through participating patients. On a few occasions, the patient refused participation but the sibling wished to participate, in which case the sibling was included independently. The CASH was also used to confirm the absence of a diagnosis of non-affective psychosis in the siblings, and absence of lifetime diagnosis of psychotic disorder in the control subjects. Control subjects were recruited using random mailings in nearby municipalities and through advertisement in newspapers. For the control subjects, the occurrence of any psychotic disorder in either the subject or any first-degree family member, assessed using the Family Interview for Genetic Studies, constituted an exclusion criterion. 
Additional exclusion criteria constituted oncologic processes, autoimmune disease, current infectious disease, cardiovascular disease and neurological disease.

\section{Amsterdam sample}

The Amsterdam sample comprised 140 patients, 125 non-psychotic siblings and 83 controls. Patients in this sample were diagnosed with schizophrenia $(n=89)$, schizophreniform disorder $(n=6)$, schizoaffective disorder $(n=19)$, delusional disorder $(n=1)$, substance-induced psychotic disorder $(n=2)$, brief psychotic disorder $(n=1)$ and psychotic disorder NOS $(n=22)$. There were 10 siblings with a diagnosis of major depressive disorder (MDD), two siblings with bipolar disorder, two siblings with developmental disorders and one sibling with anorexia nervosa. Among the controls, 3 were diagnosed with MDD and one with dysthymic disorder. Ninety families from the Amsterdam area participated. Sixty-three families contributed one patient and one sibling, 20 families contributed one patient and two siblings, three families contributed one patient and three siblings and one family contributed one patient and four siblings. Two families contributed two siblings, but no patients. One family contributed two controls. Fifty-three independent patients, 5 independent siblings and 81 independent controls participated, i.e. these individuals had no relatives in the sample.

\section{Maastricht sample}

The Maastricht sample consisted of 82 patients with psychotic disorder, 94 non-psychotic siblings of patients, and 78 controls. Of the patients, 59 were diagnosed with schizophrenia and 16 were diagnosed with schizoaffective disorder, 2 had a substance-induced psychotic disorder and 1 patient was diagnosed with brief psychotic disorder. The other patients $(n=4)$ were diagnosed with psychotic disorder not otherwise specified (NOS). In addition, 28 siblings and 16 controls had a diagnosis of MDD. A total of 50 families from Maastricht participated in the study. Thirty-one families contributed one patient and one sibling, seven families contributed one patient and two siblings, one family contributed one patient and three siblings and one family contributed two patients but no siblings. Four families contributed two siblings and one family contributed three siblings, but no patients. Five families contributed two controls. Forty-one independent patients, 35 independent siblings and 68 independent controls participated.

\section{Measures}

Body mass index (BMI)

BMI was calculated as weight in kilograms divided by height in meters squared.

Positive and negative symptoms

Psychotic symptomatology was assessed with the Positive and Negative Syndrome Scale (PANSS) (Kay et al., 1987). The scores of the individual items of the positive and negative symptom dimensions were summed. 


\section{Antipsychotic medication}

Self-report present use and type of antipsychotic medication (AP) was documented. AP was categorized by generation, i.e. first generation antipsychotics (FGA) or second and third generation antipsychotics (SGA and TGA respectively). For the analyses, SGA and TGA were combined. In case of simultaneous use of an FGA and SGA or TGA, the AP type was classified as FGA.

\section{Serum sample acquisition and processing}

Serum samples were acquired by venipuncture and were centrifuged and frozen within 24 hours. S100B was analyzed using a Liaison automated chemiluminescence analyzer according to the manufacturer's instructions (Diasorin). The lowest concentration of detection was 0.02 microgram per liter. Receiver operator curves showed best accuracy for the Liaison Sangtec 100 assay (Smit et al., 2005). All samples (from both locations) were analyzed at the same laboratory of the Radboud University Medical Centre, employing the same technique.

This study was approved by the standing ethics committees of the University Medical Centres of Utrecht (G.R.O.U.P. study), Amsterdam, Rotterdam and Maastricht. All subjects provided written informed consent in accordance with the committee's guidelines.

\section{Statistical analysis}

The strategy was analysis in one sample followed by within-study replication in the other sample. Per sample, group differences in S100B were analyzed using multilevel random regression models because of hierarchical clustering occasioned by the fact that participants were clustered in families, compromising statistical independence of the observations (Goldstein, 1987). This was done using the XTREG command in STATA (STATA corp, version 11). S100B was the dependent variable in the analyses and group (entered as both linear and dummy variables (controls $=0$, siblings $=1$ and patients $=2$ )) was the independent variable. Analyses were adjusted for the following a priori hypothesized confounders: sex, age and BMI. In addition, we tested whether S100B was not only conditional on group but also on sex, by examining group $\times$ sex interactions corrected for age and BMI. Interactions terms were evaluated by Wald test (Clayton and Hills, 1993).

To account for the clinical heterogeneity in the patient samples, the association between group and S100B was examined in two subgroups: i) patients with a narrowly defined diagnosis of schizophrenia (i.e. excluding schizoaffective disorder, schizophreniform disorder, delusional disorder and psychotic disorder NOS), and ii) patients with any psychotic disorder with PANSS positive and negative scores of fifteen or higher.

As S100B may be elevated in mood disorders (Schroeter and Steiner, 2009), sensitivity analyses were conducted, excluding controls and siblings with a history of affective disorders or other psychiatric morbidity. 
Associations between the PANSS symptom levels and S100B were investigated in the patient samples using multilevel regression analyses for the Maastricht sample (given hierarchical clustering of patients within families) and multiple regression analyses for the Amsterdam sample, with S100B as the dependent variable and symptom scores as the independent variable. In order to visualize dose-response in case of an association, PANSS symptoms scores were entered as dummy variables representing the distribution of the scores divided by its tertiles. Analyses were corrected for sex and age.

Multilevel (Maastricht sample) and multiple regression procedures (Amsterdam sample) were also used to investigate associations between present use of AP ( $0=$ no current use, $1=$ current use) and S100B. Associations between type of AP and S100B were examined by comparing FGA and SGA/TGA to no $\mathrm{AP}(0=$ no $A P, 1=F G A, 2=S G A / T G A)$. AP analyses were corrected for sex, age and BMI.

\section{RESULTS}

\section{Descriptive analyses}

Eighty-six percent of the Amsterdam patients and 77\% of the Maastricht patients was male; for the control group these figures were $69 \%$ and $32 \%$ respectively. The mean age and BMI of all groups was higher in the Maastricht sample. Patients from Amsterdam had higher negative symptom levels than those from Maastricht. Symptom levels in siblings and controls were not reported because of the high proportion of missing values in the Amsterdam sample (Table 1).

Within the samples, patients and siblings were younger than controls in Maastricht while siblings were older than patients in Amsterdam. In Maastricht, BMI was higher in patients compared to controls, but not siblings. Patients from Amsterdam had a higher BMI compared to both controls and siblings (Table 1).

Out of 140 patients from the Amsterdam sample, 112 reported the present use of an AP. Thirty-five patients used olanzapine, 31 used risperidone, 13 used clozapine, 8 used aripiprazole, 5 quetiapine, 5 haloperidol and 2 penfluridol. Two used flupenthixol, one used pimozide, and one sulpiride. Three individuals used a combination of haloperidol and clozapine, 2 used risperidone and quetiapine, 1 a combination of aripiprazole and olanzapine and 1 person used pimozide and risperidone. Two patients were unable to provide the name of the current AP. For fifteen patients, AP data was missing. In the Maastricht sample, 72 out of 82 patients currently used AP medication. Sixteen patients used risperidone, 16 used olanzapine, 12 used aripiprazole, 7 used clozapine, 5 quetiapine, 3 haloperidol and 2 used amisulpride. Two patients used zuclopenthixol and bromperidol, respectively. Two persons used a combination of clozapine and aripiprazole, 1 a combination of aripiprazole and olanzapine, 1 haloperidol and pipamperone, 1 zuclopenthixol and quetiapine, 1 clozapine and clotiapine, 1 risperidone and aripiprazole, 1 risperidone and paliperidone, and 1 a combination of risperidone, amisulpride and clotiapine. One sibling with a diagnosis of depression used a low dose of olanzapine. 
Table 1. Demographic characteristics

\begin{tabular}{|c|c|c|c|}
\hline & Controls & Siblings & Patients \\
\hline \multicolumn{4}{|c|}{ Number of participants } \\
\hline Amsterdam & 83 & 125 & 140 \\
\hline Maastricht & 78 & 94 & 82 \\
\hline \multicolumn{4}{|c|}{ Sex (male/female) } \\
\hline Amsterdam & $57 / 26$ & $57 / 68$ & $120 / 20$ \\
\hline Maastricht & $25 / 53$ & $44 / 50$ & $63 / 19$ \\
\hline \multicolumn{4}{|l|}{$\mathrm{S} 100 \mathrm{~B}(\mu \mathrm{g} / \mathrm{l})$} \\
\hline Amsterdam & $0.070 \pm 0.028$ & $0.070 \pm 0.029$ & $0.075 \pm 0.033$ \\
\hline Maastricht & $0.076 \pm 0.028$ & $0.078 \pm 0.039$ & $0.083 \pm 0.078$ \\
\hline \multicolumn{4}{|l|}{ Age } \\
\hline Amsterdam & $26.16 \pm 9.31$ & $27.03 \pm 8.11$ & $24.65 \pm 6.00$ \\
\hline Maastricht & $34.40 \pm 10.39$ & $31.61 \pm 8.59$ & $30.50 \pm 6.93$ \\
\hline \multicolumn{4}{|l|}{ BMI $\left(k g / m^{2}\right)$} \\
\hline Amsterdam & $22.41 \pm 2.47$ & $22.94 \pm 3.37$ & $23.88 \pm 3.69$ \\
\hline Maastricht & $24.39 \pm 4.74$ & $24.80 \pm 4.56$ & $25.96 \pm 4.13$ \\
\hline \multicolumn{4}{|c|}{ Positive symptoms (PANSS) } \\
\hline Amsterdam & & & $12.00 \pm 5.18$ \\
\hline Maatricht & & & $11.50 \pm 5.25$ \\
\hline \multicolumn{4}{|c|}{ Negative symptoms (PANSS) } \\
\hline Amsterdam & & & $16.21 \pm 6.83$ \\
\hline Maastricht & & & $11.17 \pm 4.66$ \\
\hline \multicolumn{4}{|c|}{ Present use of AP (yes/no) } \\
\hline Amsterdam & & & $112 / 13(15 \mathrm{NA})$ \\
\hline Maastricht & & & $72 / 10$ \\
\hline
\end{tabular}

Means \pm standard deviations reported. PANSS: Positive and negative syndrome scale; NA: not available.

\section{Associations between group and S100B}

Although the direction of the effect was positive, there was no significant association between group (linear trend) and S100B in the samples (Amsterdam: $\mathrm{B}=0.002,95 \% \mathrm{Cl}-0.002$ to $0.006, \mathrm{p}=0.377$; and Maastricht: $B=0.004,95 \% \mathrm{Cl}-0.006$ to $0.014, p=0.407$ ).

Between group (dummy variable) comparisons showed that S100B levels in patients and siblings were not significantly different from controls (Amsterdam: $P$ vs. $C: B=0.004,95 \% \mathrm{Cl}-0.005$ to 0.013 , $p=0.351$; $S$ vs. $C: B=0.000,95 \% C l-0.009$ to $0.008, p=0.926$; and Maastricht: $P$ vs. $C: B=0.008,95 \% C l$ -0.011 to $0.028, p=0.410$; $S$ vs. $C: B=0.002,95 \% \mathrm{Cl}-0.016$ to $0.021, p=0.797)$, nor was there a large or significant difference between patients and siblings (Table 2). 
Table 2. Associations between group and S100B

\begin{tabular}{|c|c|c|c|}
\hline & S vs. C & P vs. C & P vs. S \\
\hline & B (95\% Cl), p-value & B (95\% Cl), p-value & B (95\% Cl), p-value \\
\hline \multicolumn{4}{|c|}{ Group (AD +) } \\
\hline Amsterdam & $\begin{array}{l}0.000(-0.009-0.008) \\
p=0.926\end{array}$ & $\begin{array}{l}0.004(-0.005-0.013) \\
p=0.351\end{array}$ & $\begin{array}{l}0.005(-0.003-0.012) \\
p=0.207\end{array}$ \\
\hline Maastricht & $\begin{array}{l}0.002(-0.016-0.021) \\
p=0.797\end{array}$ & $\begin{array}{l}0.008(-0.011-0.028) \\
p=0.410\end{array}$ & $\begin{array}{l}0.006(-0.011-0.023) \\
p=0.504\end{array}$ \\
\hline \multicolumn{4}{|c|}{ Group (AD -) } \\
\hline Amsterdam & $\begin{array}{l}0.000(-0.009-0.009) \\
p=0.995\end{array}$ & $\begin{array}{l}0.004(-0.005-0.013) \\
p=0.396\end{array}$ & $\begin{array}{l}0.004(-0.003-0.011) \\
p=0.254\end{array}$ \\
\hline Maastricht & $\begin{array}{l}0.001(-0.020-0.023) \\
p=0.897\end{array}$ & $\begin{array}{l}0.009(-0.013-0.030) \\
p=0.436\end{array}$ & $\begin{array}{l}0.007(-0.013-0.027) \\
p=0.482\end{array}$ \\
\hline \multicolumn{4}{|c|}{ Group (SCZ only) } \\
\hline Amsterdam & $\begin{array}{l}-0.001(-0.010-0.008) \\
p=0.821\end{array}$ & $\begin{array}{l}0.003(-0.006-0.013) \\
p=0.473\end{array}$ & $\begin{array}{l}0.004(-0.003-0.012) \\
p=0.268\end{array}$ \\
\hline Maastricht & $\begin{array}{l}0.001(-0.014-0.016) \\
p=0.875\end{array}$ & $\begin{array}{l}-0.001(-0.019-0.017) \\
p=0.926\end{array}$ & $\begin{array}{l}-0.002(-0.017-0.013) \\
p=0.787\end{array}$ \\
\hline \multicolumn{4}{|c|}{ Group (PANSS pos $\geq 15$ ) } \\
\hline Amsterdam & $\begin{array}{l}-0.002(-0.011-0.006) \\
p=0.598\end{array}$ & $\begin{array}{l}0.008(-0.003-0.020) \\
p=0.165\end{array}$ & $\begin{array}{l}0.010(0.000-0.020) \\
p=0.040\end{array}$ \\
\hline Maastricht & $\begin{array}{l}0.003(-0.010-0.015) \\
p=0.702\end{array}$ & $\begin{array}{l}-0.005(-0.026-0.017) \\
p=0.683\end{array}$ & $\begin{array}{l}-0.007(-0.027-0.013) \\
p=0.487\end{array}$ \\
\hline \multicolumn{4}{|c|}{ Group (PANSS neg $\geq 15$ ) } \\
\hline Amsterdam & $\begin{array}{l}-0.001(-0.010-0.008) \\
p=0.850\end{array}$ & $\begin{array}{l}0.010(0.000-0.020) \\
p=0.051\end{array}$ & $\begin{array}{l}0.011(0.002-0.020) \\
p=0.016\end{array}$ \\
\hline Maastricht & $\begin{array}{l}0.002(-0.011-0.015) \\
p=0.719\end{array}$ & $\begin{array}{l}0.011(-0.012-0.034) \\
p=0.344\end{array}$ & $\begin{array}{l}0.009(-0.012-0.029) \\
p=0.410\end{array}$ \\
\hline
\end{tabular}

Bs represent the regression coefficients of the multilevel regression analyses.

95\% Cl: 95\% confidence interval.

S vs. C: sibling versus control; P vs. C: patient versus control; P vs. S: patient versus sibling.

$A D+$ : affective disorders in controls and siblings included; $A D$-: affective disorders in controls and siblings excluded; SCZ: schizophrenia only in patient groups; PANSS: Positive and negative syndrome scale; PANSS pos $\geq 15$ : PANSS positive symptom level above or equal to 15 in patient groups; PANSS neg $\geq 15$ : PANSS negative symptom level above or equal to 15 in patient groups.

\section{Association between group and S100B: subgroup analyses}

\section{Narrow schizophrenia diagnosis only}

Excluding patients with a diagnosis other than schizophrenia altered the pattern of effect sizes in some instances, but none of the between-group comparisons became statistically significant (Table 2), nor the effect of group as a linear trend variable (Amsterdam: $B=0.001,95 \% \mathrm{Cl}-0.004$ to 0.006 , $\mathrm{p}=0.696$; and Maastricht: $\mathrm{B}=0.001,95 \% \mathrm{Cl}-0.008$ to $0.010, \mathrm{p}=0.806$ ). 
All psychotic disorders with PANSS scores $\geq 15$ (on positive and negative subscales respectively) At a cut-off of fifteen in PANSS positive symptom levels, 33 patients remained in the Amsterdam sample and 14 remained in the Maastricht sample. There were no significant associations between $\mathrm{S100B}$ and group (linear trend) (Amsterdam: $B=0.002,95 \% \mathrm{Cl}-0.004$ to $0.008, p=0.539$; and Maastricht: $B=0.000$, $95 \% \mathrm{Cl}-0.010$ to $0.010, \mathrm{p}=0.966$ ). In the between-group comparison, patients had significantly higher S100B levels compared to siblings in the Amsterdam sample, although patients and siblings were not significantly different from controls. In the Maastricht sample, there were no large or significant associations between group and S100B, with an opposite direction of effect sizes (Table 2).

At a cut-off of fifteen in PANSS negative symptom levels, 72 Amsterdam and 13 Maastricht patients remained in the analyses. There was no significant association between $\mathrm{S100B}$ and group as a linear trend variable (Amsterdam: $\mathrm{B}=0.005,95 \% \mathrm{Cl} 0.000$ to $0.010, \mathrm{p}=0.076$; and Maastricht: $\mathrm{B}=0.005,95 \% \mathrm{Cl}$ -0.005 to $0.015, p=0.349$ ). However, between-group comparisons showed that patients in Amsterdam had significantly higher S100B levels than siblings $(B=0.011,95 \% \mathrm{Cl} 0.002$ to $0.020, p=0.016)$, and controls - at trend-level $(B=0.010,95 \% \mathrm{Cl} 0.000$ to $0.020, p=0.051)$. This was not replicated in the Maastricht sample (Table 2).

\section{Exclusion of affective disorders and other psychiatric morbidity in siblings and controls}

The exclusion of controls and siblings with a history of affective disorder or other psychiatric morbidity did not affect the findings when using the original patient samples, or any of the above described sub-analyses (Table 2).

\section{Associations between symptomatology and S100B}

In patients (all diagnoses), negative symptoms were positively associated with $\mathrm{S100B}$ in the Amsterdam sample ( $B=0.001,95 \% \mathrm{Cl} 0.000$ to $0.002, p=0.005)$, but not in the Maastricht sample $(B=0.002,95 \% C$ l -0.002 to $0.005, p=0.308$ ). In the Amsterdam sample, progressively higher negative symptom levels were associated with progressively higher S100B levels, although only the highest negative symptom level reached statistical significance (high vs. low symptom group: $B=0.016,95 \% \mathrm{Cl} 0.003$ to 0.030 , $p=0.020$; intermediate vs. low symptom group: $B=0.010,95 \% \mathrm{Cl}-0.003$ to $0.024, p=0.127$ ).

There was no large or statistically significant association between positive symptoms and $\mathrm{S100B}$ in the Amsterdam or Maastricht sample $(B=0.001,95 \% \mathrm{Cl} 0.000$ to $0.002, p=0.098$; and $B=-0.001,95 \% \mathrm{Cl}$ -0.004 to $0.002, p=0.483$ respectively).

\section{Associations between antipsychotic medication and S100B}

In patients, the present use of AP medication was not significantly associated with $\mathrm{S100B}$ (Amsterdam: $\mathrm{B}=-0.003,95 \% \mathrm{Cl}-0.022$ to $0.017, \mathrm{p}=0.781$; Maastricht: $\mathrm{B}=0.027,95 \% \mathrm{Cl}-0.024$ to $0.078, \mathrm{p}=0.302$ ). Similarly, there was no effect of type of AP, comparing FGA and SGA/TGA users to AP-free patients and comparing FGA to SGA/TGA users (Table 3). 
Table 3. Associations between AP medication and S100B

\begin{tabular}{llll}
\hline & FGA vs. no AP & SGA/TGA vs. no AP & SGA/TGA vs. FGA \\
\hline Patients & & & \\
Amsterdam & $0.005(-0.020-0.029)$, & $-0.004(-0.024-0.015)$, & $-0.009(-0.028-0.010)$, \\
& $p=0.713$ & $p=0.659$ & $p=0.335$ \\
Maastricht & $-0.001(-0.076-0.073)$, & $0.030(-0.021-0.081)$, & $0.031(-0.029-0.092)$, \\
& $p=0.970$ & $p=0.248$ & $p=0.309$ \\
\hline
\end{tabular}

Bs $(95 \%$ confidence interval) and $p$-value reported. Bs represent the regression coefficients of the multilevel regression analyses.

AP: antipsychotic medication; FGA: first generation AP; SGA/TGA: second and third generation AP.

\section{Group $\times$ sex interactions and S100B}

There was no evidence for significant group $\times$ sex interactions in either sample (Amsterdam: $x^{2}=0.35$, $p=0.839 ;$ Maastricht: $x^{2}=1.45, p=0.484$ ).

\section{DISCUSSION}

Contrary to the hypothesis, there was no statistically significant association between group and S100B. Serum S100B levels in patients and siblings were not different from controls. In the Amsterdam sample, there was a significant positive association between negative symptoms and S100B, which was not replicated in the Maastricht sample. There was no large or significant association between S100B and positive symptoms or the present use or type of AP in either sample.

\section{Findings}

(Familial) risk of psychotic disorder and S100B

We did not detect significantly elevated S100B in patients, in disagreement with a range of previous small studies. Other investigations suggesting an absence of elevated S100B in schizophrenia are limited. One study found unaltered S100B levels in unmedicated patients compared to controls, although S100B was elevated in patients who had been treated with AP for 3 weeks compared to unmedicated patients and controls (Schroeter et al., 2003). In another study, a small sample of patients with schizophrenia had higher levels of S100B in cerebrospinal fluid (CSF) but not serum (after Bonferroni correction) (Steiner et al., 2006). There is one study reporting reduced S100B levels (Gattaz et al., 2000). Although the absence of elevated S100B in patients does not agree with most literature, the current results provided a replication in two samples that were considerably larger than earlier work (except for two studies (Rothermundt et al., 2004b; Zhang et al., 2010)). Another difference between the current and other studies concerns the patient population. The current samples comprised mainly outpatients, generally not in an acute stage of the disorder, with a mean illness duration of $<10$ years, 
whereas the majority of previous work was conducted in hospitalized patients. The evidence to date may thus be suggestive of S100B elevation in inpatient populations with presumably more severe symptomatology. Furthermore, a considerable proportion of the patients in the current study had a psychotic disorder other than schizophrenia, while previous studies were conducted specifically in schizophrenic patients. However, the diagnosis of schizophrenia does not refer to a natural illness type. The different diagnostic categories in the DSM-IV chapter of psychotic disorders likely reflect phenotypic diversity related to a shared liability. In addition, the sensitivity analyses (excluding patients with a diagnosis of psychotic disorder other than schizophrenia) did not provide evidence for differences between groups. It should further be noted that serological and histological S100B alterations have been associated with affective disorders (Gos et al., 2013; Schroeter and Steiner, 2009). In the current sample in- or exclusion of all psychiatric morbidity in siblings and controls yielded the same results: the absence of association between serum S100B and risk of psychotic disorder.

This is the first study to investigate serum S100B as a marker of familial risk of psychotic disorder. The use of non-psychotic siblings in the study design makes it possible to establish the effect of shared familial risk of psychotic disorder, without confounding by disease-related factors. The data did not suggest that serum S100B is an intermediate phenotype for psychotic disorder. The absence of S100B alterations in our relatively stable patient and non-psychotic sibling populations, in combination with the previously reported increased S100B levels in hospitalized patient populations, may suggest that S100B elevations reflect fluctuating disease processes in certain patient populations, i.e. that they are related to the clinical "state". This reasoning may be underscored by the (albeit weak) association between S100B and negative symptoms in the Amsterdam sample. Alternatively, it may be that previous work reporting positive associations was influenced by publication/reporting bias, particularly given the suggestion of excess significance bias in the literature, affecting the representation of true associations between psychiatric phenotypes and biological measures (loannidis, 2011).

\section{Symptomatology and S100B}

Narrowing down the diagnosis of schizophrenia did not affect the pattern of findings. In addition, patients in the Amsterdam sample with more severe positive symptomatology (PANSS scores $\geq 15$ ) had higher S100B levels compared to siblings but not compared to controls, while patients with more severe negative symptoms had higher S100B levels compared to both siblings and controls (at trend-level), regardless of diagnosis. This was not replicated in the Maastricht sample. A cut-off of 15 for the PANSS positive and negative symptom levels is clinically quite conservative. A higher cut-off, however, would have further reduced the samples size. These findings suggest that the absence of S100B elevation was not due to diagnostic heterogeneity.

To examine whether S100B elevation may be associated with higher symptom levels in patient populations, main effects of symptomatology on S100B were assessed. The positive association between negative symptoms and S100B, as found in the Amsterdam sample, has been reported previously (Ling et al., 2007; Rothermundt et al., 2004a; Rothermundt et al., 2001; Schroeter et al., 
2003). Despite the statistical significance of this finding, the clinical relevance is debatable given the small effect size. Furthermore, the association between negative symptoms and S100B levels was not replicated in the Maastricht sample, although size and direction of the association were similar. Patients in Amsterdam had higher negative symptom levels than patients in Maastricht, possibly due to the higher proportion of men in the Amsterdam sample. The negative symptom level in the Maastricht patient sample was also quite low compared to other studies that did find an association using the PANSS.

A recent review (Yelmo-Cruz et al., 2013) expressed the opinion that S100B studies have been tainted by bias due to selection of patients with high negative symptom levels. Nevertheless, certain explanations for a true positive association between S100B and negative symptoms could be conceived. First, neuroimaging work (Streitburger et al., 2012) has confirmed white matter tracts as sites of profuse $5100 \mathrm{~B}$ expression (by glia and oligodendrocytes) and white matter abnormalities have been associated with negative symptoms (Makris et al., 2010; Nakamura et al., 2012; Szeszko et al., 2008; Voineskos et al., 2013; Wolkin et al., 2003). Second, S100B may reflect a compensatory mechanism instead of a causative process, cueing in recovery at a stage of illness when positive symptoms have begun to remit and negative symptoms become more prominent.

\section{AP medication and S100B}

Previously reported effects of AP on S100B have been inconsistent. A decline in S100B after several weeks of AP treatment has been reported (Rothermundt et al., 2001; Schroeter et al., 2003), and in vitro work by Steiner (Steiner et al., 2010c) also suggests that S100B levels are normalized by AP. However, a longitudinal study showed that S100B remained increased after 24 weeks of treatment (Rothermundt et al., 2004b). In other studies, medication-free patients with schizophrenia had higher serum S100B than healthy controls (Lara et al., 2001) and drug-naïve first-episode patients had significantly higher serum S100B levels than chronic patients on long-term AP (Zhang et al., 2010), with the latter group showing S100B elevation compared to healthy controls. These studies show that S100B elevation cannot be ascribed to AP. A recent meta-analysis did not find an association between S100B in schizophrenia and AP treatment (Schroeter et al., 2009), in line with the current findings.

\section{Methodological considerations}

The strength of the present study was that it comprised two large patient-sibling-control samples, allowing for independent replication. As an additional validation, we repeated the analyses combining the Amsterdam and Maastricht samples to increase power. In the combined sample, the absence of a significant positive association between S100B and group was upheld. The association between negative symptoms and S100B in patients (which was only present in the Amsterdam sample) was not significant ( $p=0.081$ ) in the combined sample (results available on request).

Certain limitations should be addressed. There is debate about the specificity of S100B as a marker of CNS pathology as S100B is expressed in other cells and tissues in physiological circumstances, e.g. 
adipocytes, chondrocytes, lymphocytes and melanocytes (Donato et al., 2013; Donato et al., 2009; Steiner et al., 2010b), as well as in pathological conditions, e.g. cardiomyocytes after infarction (Donato et al., 2013; Donato and Heizmann, 2010; Donato et al., 2009). One way to circumvent this issue is to obtain S100B levels in the CSF. In a comparative serum/CSF study, serum S100B was found to mirror CSF concentrations (Rothermundt et al., 2004a). Corroborating evidence comes from a study reporting that extracerebral sources of S100B did not alter S100B serum levels (Pham et al., 2010). Thus, despite the diverse origins of serum S100B, evidence suggests that serum S100B is correlated with CSF S100B. As Steiner and colleagues found that S100B is associated with BMI, we adjusted for BMI (Steiner et al., 2010b). In contrast, Pham and colleagues did not find an association between BMI and S100B (Pham et al., 2010). The pattern of our findings was unaltered when BMI was removed as a covariate from the analysis. To clarify a potential link to insulin resistance (Steiner et al., 2010a; Steiner et al., 2010d), it may be useful to measure $\mathrm{HbA1c}$ in future studies.

\section{Conclusions}

Our findings suggest the following: i) S100B is not an intermediate phenotype of psychotic disorder; and ii) S100B is not elevated in the "general population" of individuals with psychotic disorder. 


\section{REFERENCES}

Andreasen, N.C., Flaum, M., Arndt, S., 1992. The Comprehensive Assessment of Symptoms and History (CASH). An instrument for assessing diagnosis and psychopathology. Arch Gen Psychiatry 49(8), 615-623.

American Psychiatric Association, 2000. Diagnostic and statistical manual of mental disorders, 4 ed. American Psychiatric Press, Washington, DC.

Camchong, J., Lim, K.O., Sponheim, S.R., Macdonald, A.W., 2009. Frontal white matter integrity as an endophenotype for schizophrenia: diffusion tensor imaging in monozygotic twins and patients' nonpsychotic relatives. Front Hum Neurosci 3, 35.

Clark, K.A., Nuechterlein, K.H., Asarnow, R.F., Hamilton, L.S., Phillips, O.R., Hageman, N.S., Woods, R.P., Alger, J.R., Toga, A.W., Narr, K.L., 2011. Mean diffusivity and fractional anisotropy as indicators of disease and genetic liability to schizophrenia. J Psychiatr Res 45(7), 980-988.

Clayton, D., Hills, M., 1993. Statistical Models in Epidemiology. Oxford University Press, Oxford.

Donato, R., Cannon, B.R., Sorci, G., Riuzzi, F., Hsu, K., Weber, D.J., Geczy, C.L., 2013. Functions of S100 proteins. Curr Mol Med 13(1), 24-57.

Donato, R., Heizmann, C.W., 2010. S100B Protein in the Nervous System and Cardiovascular Apparatus in Normal and Pathological Conditions. Cardiovasc Psychiatry Neurol 2010, 929712.

Donato, R., Sorci, G., Riuzzi, F., Arcuri, C., Bianchi, R., Brozzi, F., Tubaro, C., Giambanco, I., 2009. S100B's double life: intracellular regulator and extracellular signal. Biochim Biophys Acta 1793(6), 1008-1022.

Fernandez-Egea, E., Bernardo, M., Parellada, E., Justicia, A., Garcia-Rizo, C., Esmatjes, E., Conget, I., Kirkpatrick, B., 2008a. Glucose abnormalities in the siblings of people with schizophrenia. Schizophr Res 103(1-3), 110-113.

Fernandez-Egea, E., Miller, B., Bernardo, M., Donner, T., Kirkpatrick, B., 2008b. Parental history of type 2 diabetes in patients with nonaffective psychosis. Schizophr Res 98(1-3), 302-306.

Gattaz, W.F., Lara, D.R., Elkis, H., Portela, L.V., Goncalves, C.A., Tort, A.B., Henna, J., Souza, D.O., 2000. Decreased S100beta protein in schizophrenia: preliminary evidence. Schizophr Res 43(2-3), 91-95.

Goldstein, H., 1987. Multilevel Models in Educational and Social Research. Griffin, London.

Gos, T., Schroeter, M.L., Lessel, W., Bernstein, H.G., Dobrowolny, H., Schiltz, K., Bogerts, B., Steiner, J., 2013. S100Bimmunopositive astrocytes and oligodendrocytes in the hippocampus are differentially afflicted in unipolar and bipolar depression: A postmortem study. J Psychiatr Res.

Guest, P.C., Wang, L., Harris, L.W., Burling, K., Levin, Y., Ernst, A., Wayland, M.T., Umrania, Y., Herberth, M., Koethe, D., van Beveren, J.M., Rothermundt, M., McAllister, G., Leweke, F.M., Steiner, J., Bahn, S., 2010. Increased levels of circulating insulin-related peptides in first-onset, antipsychotic naive schizophrenia patients. Mol Psychiatry 15(2), 118-119.

Habets, P., Marcelis, M., Gronenschild, E., Drukker, M., van Os, J., 2011. Reduced cortical thickness as an outcome of differential sensitivity to environmental risks in schizophrenia. Biol Psychiatry 69(5), 487-494.

Hao, Y., Yan, Q., Liu, H., Xu, L., Xue, Z., Song, X., Kaneko, Y., Jiang, T., Liu, Z., Shan, B., 2009. Schizophrenia patients and their healthy siblings share disruption of white matter integrity in the left prefrontal cortex and the hippocampus but not the anterior cingulate cortex. Schizophr Res 114(1-3), 128-135.

loannidis, J.P., 2011. Excess significance bias in the literature on brain volume abnormalities. Arch Gen Psychiatry 68(8), 773-780.

Kay, S.R., Fiszbein, A., Opler, L.A., 1987. The positive and negative syndrome scale (PANSS) for schizophrenia. Schizophr Bull 13(2), 261-276.

Knochel, C., Oertel-Knochel, V., Schonmeyer, R., Rotarska-Jagiela, A., van de Ven, V., Prvulovic, D., Haenschel, C., Uhlhaas, P., Pantel, J., Hampel, H., Linden, D.E., 2012. Interhemispheric hypoconnectivity in schizophrenia: fiber integrity and volume differences of the corpus callosum in patients and unaffected relatives. Neuroimage 59(2), 926-934. 
Korver, N., Quee, P.J., Boos, H.B., Simons, C.J., de Haan, L., 2012. Genetic Risk and Outcome of Psychosis (GROUP), a multi-site longitudinal cohort study focused on gene-environment interaction: objectives, sample characteristics, recruitment and assessment methods. International journal of methods in psychiatric research 21(3), 205-221.

Lara, D.R., Gama, C.S., Belmonte-de-Abreu, P., Portela, L.V., Goncalves, C.A., Fonseca, M., Hauck, S., Souza, D.O., 2001. Increased serum S100B protein in schizophrenia: a study in medication-free patients. J Psychiatr Res 35(1), 11-14.

Ling, S.H., Tang, Y.L., Jiang, F., Wiste, A., Guo, S.S., Weng, Y.Z., Yang, T.S., 2007. Plasma S-100B protein in Chinese patients with schizophrenia: comparison with healthy controls and effect of antipsychotics treatment. J Psychiatr Res 41(1-2), 36-42.

Liu, J., Shi, Y., Tang, J., Guo, T., Li, X., Yang, Y., Chen, Q., Zhao, X., He, G., Feng, G., Gu, N., Zhu, S., Liu, H., He, L., 2005. SNPs and haplotypes in the S100B gene reveal association with schizophrenia. Biochem Biophys Res Commun 328(1), 335-341.

Makris, N., Seidman, L.J., Ahern, T., Kennedy, D.N., Caviness, V.S., Tsuang, M.T., Goldstein, J.M., 2010. White matter volume abnormalities and associations with symptomatology in schizophrenia. Psychiatry Res 183(1), 21-29.

Michetti, F., Corvino, V., Geloso, M.C., Lattanzi, W., Bernardini, C., Serpero, L., Gazzolo, D., 2012. The S100B protein in biological fluids: more than a lifelong biomarker of brain distress. J Neurochem 120(5), 644-659.

Mukherjee, S., Schnur, D.B., Reddy, R., 1989. Family history of type 2 diabetes in schizophrenic patients. Lancet $1(8636), 495$.

Nakamura, K., Kawasaki, Y., Takahashi, T., Furuichi, A., Noguchi, K., Seto, H., Suzuki, M., 2012. Reduced white matter fractional anisotropy and clinical symptoms in schizophrenia: a voxel-based diffusion tensor imaging study. Psychiatry Res 202(3), 233-238.

Pham, N., Fazio, V., Cucullo, L., Teng, Q., Biberthaler, P., Bazarian, J.J., Janigro, D., 2010. Extracranial sources of S100B do not affect serum levels. PLoS One 5(9).

Pillai, A., Buckley, P.F., 2012. Reliable biomarkers and predictors of schizophrenia and its treatment. Psychiatr Clin North Am 35(3), 645-659.

Rothermundt, M., Ahn, J.N., Jorgens, S., 2009. S100B in schizophrenia: an update. Gen Physiol Biophys 28 Spec No Focus, F76-81.

Rothermundt, M., Falkai, P., Ponath, G., Abel, S., Burkle, H., Diedrich, M., Hetzel, G., Peters, M., Siegmund, A., Pedersen, A., Maier, W., Schramm, J., Suslow, T., Ohrmann, P., Arolt, V., 2004a. Glial cell dysfunction in schizophrenia indicated by increased S100B in the CSF. Mol Psychiatry 9(10), 897-899.

Rothermundt, M., Missler, U., Arolt, V., Peters, M., Leadbeater, J., Wiesmann, M., Rudolf, S., Wandinger, K.P., Kirchner H., 2001. Increased S100B blood levels in unmedicated and treated schizophrenic patients are correlated with negative symptomatology. Mol Psychiatry 6(4), 445-449.

Rothermundt, M., Ohrmann, P., Abel, S., Siegmund, A., Pedersen, A., Ponath, G., Suslow, T., Peters, M., Kaestner, F., Heindel, W., Arolt, V., Pfleiderer, B., 2007. Glial cell activation in a subgroup of patients with schizophrenia indicated by increased S100B serum concentrations and elevated myo-inositol. Prog Neuropsychopharmacol Biol Psychiatry 31(2), 361-364.

Rothermundt, M., Ponath, G., Glaser, T., Hetzel, G., Arolt, V., 2004b. S100B serum levels and long-term improvement of negative symptoms in patients with schizophrenia. Neuropsychopharmacology 29(5), 1004-1011.

Schroeter, M.L., Abdul-Khaliq, H., Fruhauf, S., Hohne, R., Schick, G., Diefenbacher, A., Blasig, I.E., 2003. Serum S100B is increased during early treatment with antipsychotics and in deficit schizophrenia. Schizophr Res 62(3), 231236.

Schroeter, M.L., Abdul-Khaliq, H., Krebs, M., Diefenbacher, A., Blasig, I.E., 2009. Neuron-specific enolase is unaltered whereas S100B is elevated in serum of patients with schizophrenia--original research and meta-analysis. Psychiatry Res 167(1-2), 66-72.

Schroeter, M.L., Steiner, J., 2009. Elevated serum levels of the glial marker protein S100B are not specific for schizophrenia or mood disorders. Mol Psychiatry 14(3), 235-237. 
Sen, J., Belli, A., 2007. S100B in neuropathologic states: the CRP of the brain? J Neurosci Res 85(7), 1373-1380.

Smit, L.H., Korse, C.M., Bonfrer, J.M., 2005. Comparison of four different assays for determination of serum S-100B. Int J Biol Markers 20(1), 34-42.

Steiner, J., Bernstein, H.G., Bielau, H., Farkas, N., Winter, J., Dobrowolny, H., Brisch, R., Gos, T., Mawrin, C., Myint, A.M., Bogerts, B., 2008. S100B-immunopositive glia is elevated in paranoid as compared to residual schizophrenia: a morphometric study. J Psychiatr Res 42(10), 868-876.

Steiner, J., Bielau, H., Bernstein, H.G., Bogerts, B., Wunderlich, M.T., 2006. Increased cerebrospinal fluid and serum levels of S100B in first-onset schizophrenia are not related to a degenerative release of glial fibrillar acidic protein, myelin basic protein and neurone-specific enolase from glia or neurones. J Neurol Neurosurg Psychiatry 77(11), 1284-1287.

Steiner, J., Myint, A.M., Schiltz, K., Westphal, S., Bernstein, H.G., Walter, M., Schroeter, M.L., Schwarz, M.J., Bogerts, B., 2010a. S100B Serum Levels in Schizophrenia Are Presumably Related to Visceral Obesity and Insulin Resistance. Cardiovasc Psychiatry Neurol 2010, 480707.

Steiner, J., Schiltz, K., Walter, M., Wunderlich, M.T., Keilhoff, G., Brisch, R., Bielau, H., Bernstein, H.G., Bogerts, B., Schroeter, M.L., Westphal, S., 2010b. S100B serum levels are closely correlated with body mass index: an important caveat in neuropsychiatric research. Psychoneuroendocrinology 35(2), 321-324.

Steiner, J., Schroeter, M.L., Schiltz, K., Bernstein, H.G., Muller, U.J., Richter-Landsberg, C., Muller, W.E., Walter, M., Gos, T., Bogerts, B., Keilhoff, G., 2010c. Haloperidol and clozapine decrease S100B release from glial cells. Neuroscience 167(4), 1025-1031.

Steiner, J., Walter, M., Guest, P., Myint, A.M., Schiltz, K., Panteli, B., Brauner, M., Bernstein, H.G., Gos, T., Herberth, M., Schroeter, M.L., Schwarz, M.J., Westphal, S., Bahn, S., Bogerts, B., 2010d. Elevated S100B levels in schizophrenia are associated with insulin resistance. Mol Psychiatry 15(1), 3-4.

Streitburger, D.P., Arelin, K., Kratzsch, J., Thiery, J., Steiner, J., Villringer, A., Mueller, K., Schroeter, M.L., 2012. Validating Serum S100B and Neuron-Specific Enolase as Biomarkers for the Human Brain - A Combined Serum, Gene Expression and MRI Study. PLoS One 7(8), e43284.

Szeszko, P.R., Robinson, D.G., Ashtari, M., Vogel, J., Betensky, J., Sevy, S., Ardekani, B.A., Lencz, T., Malhotra, A.K., McCormack, J., Miller, R., Lim, K.O., Gunduz-Bruce, H., Kane, J.M., Bilder, R.M., 2008. Clinical and neuropsychological correlates of white matter abnormalities in recent onset schizophrenia. Neuropsychopharmacology 33(5), 976-984.

van Beveren, N.J., Hoogendijk, W.J., 2011. Clinical utility of serum biomarkers for major psychiatric disorders. Int Rev Neurobiol 101, 351-374.

van Beveren, N.J., van der Spelt, J.J., de Haan, L., Fekkes, D., 2006. Schizophrenia-associated neural growth factors in peripheral blood. A review. Eur Neuropsychopharmacol 16(7), 469-480.

van Nimwegen, L.J., Storosum, J.G., Blumer, R.M., Allick, G., Venema, H.W., de Haan, L., Becker, H., van Amelsvoort, T., Ackermans, M.T., Fliers, E., Serlie, M.J., Sauerwein, H.P., 2008. Hepatic insulin resistance in antipsychotic naive schizophrenic patients: stable isotope studies of glucose metabolism. J Clin Endocrinol Metab 93(2), 572-577.

Voineskos, A.N., Foussias, G., Lerch, J., Felsky, D., Remington, G., Rajji, T.K., Lobaugh, N., Pollock, B.G., Mulsant, B.H., 2013. Neuroimaging evidence for the deficit subtype of schizophrenia. JAMA Psychiatry 70(5), 472-480.

Wiesmann, M., Wandinger, K.P., Missler, U., Eckhoff, D., Rothermundt, M., Arolt, V., Kirchner, H., 1999. Elevated plasma levels of S-100b protein in schizophrenic patients. Biol Psychiatry 45(11), 1508-1511.

Wolkin, A., Choi, S.J., Szilagyi, S., Sanfilipo, M., Rotrosen, J.P., Lim, K.O., 2003. Inferior frontal white matter anisotropy and negative symptoms of schizophrenia: a diffusion tensor imaging study. Am J Psychiatry 160(3), 572-574.

Yelmo-Cruz, S., Morera-Fumero, A.L., Abreu-Gonzalez, P., 2013. S100B and schizophrenia. Psychiatry Clin Neurosci 67(2), 67-75.

Zhai, J., Cheng, L., Dong, J., Shen, Q., Zhang, Q., Chen, M., Gao, L., Chen, X., Wang, K., Deng, X., Xu, Z., Ji, F., Liu, C., Li, J., Dong, Q., Chen, C., 2012. S100B gene polymorphisms predict prefrontal spatial function in both schizophrenia patients and healthy individuals. Schizophr Res 134(1), 89-94.

Zhang, X.Y., Xiu, M.H., Song, C., Chen da, C., Wu, G.Y., Haile, C.N., Kosten, T.A., Kosten, T.R., 2010. Increased serum S100B in never-medicated and medicated schizophrenic patients. J Psychiatr Res 44(16), 1236-1240. 
a Departments of Psychiatry and Psychology, Maastricht University Medical Centre, PO Box 616 (Vijv1), 6200 MD, Maastricht, the Netherlands

$b$ Departments of Neurology and Laboratory Medicine, Radboud University Medical Centre, Nijmegen, the Netherlands

c Donders Institute for Brain, Cognition and Behaviour, Nijmegen, the Netherlands

d Central Diagnostic Laboratory, Maastricht University Medical Centre, Maastricht, the Netherlands

e King's College London, King's Health Partners, Department of Psychosis Studies, Institute of Psychiatry, London, UK

f Institute for Mental Health Care Eindhoven (GGzE), Eindhoven, the Netherlands 


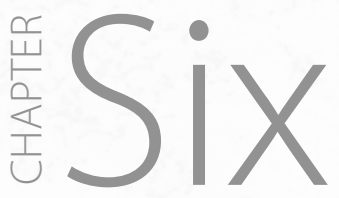

Replicated evidence of absence of association between serum S100B and (risk of) psychotic disorder

\author{
C. van der Leeuwa MD \\ S. Peeters ${ }^{\mathrm{a}} \mathrm{MSC}$ \\ E. Gronenschild PhDa \\ S. Michielse MSC ${ }^{\mathrm{a}}$ \\ M.M. Verbeek ${ }^{b, c}$ PhD MSc \\ P. Menheered PhD \\ J. van $\mathrm{Os}^{\mathrm{a}, \mathrm{e}} \mathrm{MD} \mathrm{PhD}$ \\ M. Marcelis ${ }^{\mathrm{a}, \mathrm{f}} \mathrm{MD}$ PhD; for G.R.O.U.P.
}




\section{ABSTRACT}

Background $\mathrm{S100B}$ is a protein with dose-dependent neurotrophic and neurotoxic effects. Whether S100B in psychotic disorder mirrors pathophysiological mechanisms (which elicit exacerbation of disease) or compensatory action is unclear, as is its validity as a proxy marker for brain status. Insight may be gained by examining associations between serum S100B and indices of grey matter (cortical thickness (CT)) and white matter (fractional anisotropy (FA)), in relation to the absence or presence of (increased risk of) psychotic disorder.

Methods Blood samples and cerebral magnetic resonance imaging (MRI) scans were acquired in 32 patients with a psychotic disorder, 44 non-psychotic siblings of patients with a psychotic disorder and 26 controls. Interactions between S100B and group were examined in separate models of CT and FA measures with multilevel regression analyses. All analyses were adjusted for age, body mass index (BMI), scan sequence and sex.

Results Neither CT nor FA were associated with S100B. There were no significant S100B $\times$ group interactions ( $\left.C T: x^{2}=0.59, p=0.743 ; F A: X 3.72=2, p=0.156\right)$.

Conclusions No evidence was present for S100B as a proxy marker of grey or white matter status. The association between S100B and brain measures was not moderated by psychosis risk. 


\section{INTRODUCTION}

S100B is a protein with dose-dependent neurotrophic and neurotoxic effects. Previously, we investigated serum protein S100B as a marker of familial risk of psychotic disorder. We did not find S100B elevation in unaffected siblings of patients with a psychotic disorder. Nor was S100B elevated in patients with a psychotic disorder in our samples (including patients generally not in an acute stage of the disorder, with a mean illness duration < 10 years) (van der Leeuw et al., 2013). The absence of S100B elevation in patients and siblings in our study, in combination with the previously reported positive findings in predominantly hospitalized patients (with more severe psychopathology), suggests that S100B elevations may reflect non-remitted illness phase processes in certain patient populations. A recent systematic review and meta-analysis (Aleksovska et al., 2014), which included our study, showed a significant increase in S100B in patients with schizophrenia which remained present after subgroup analyses to account for clinical and methodological heterogeneity (although heterogeneity remained high in the subgroup analyses). Whether S100B in psychotic disorder mirrors pathophysiological mechanisms (which elicit exacerbation of disease) or compensatory action is unclear.

Aleksovska and colleagues suggest that $\mathrm{S100B}$ is a candidate biomarker of neuroplasticity although there is little literature to illustrate this. One study associated S100B overexpression in transgenic mice with neurogenesis in the hippocampus in stressful conditions (Buschert et al., 2013). In other animal studies, posttraumatic intraventricular infusion of S100B has been shown to benefit hippocampal neurogenesis and cognitive function (Kleindienst et al., 2004; Kleindienst et al., 2005). Human studies are at an earlier stage. Streitbürger and colleagues (Streitburger et al., 2012) were the first to test the validity of $\mathrm{S100B}$ as a peripheral marker for the human brain. Their study demonstrated that $\mathrm{S} 100 \mathrm{~B}$ was negatively correlated with fractional anisotropy (FA) and positively correlated with radial diffusivity in healthy females, possibly signaling diminished fiber directionality and demyelination. No associations between S100B and grey matter indices were found.

To date, the validity of S100B as a proxy marker for white and grey matter status has not been investigated in patients with a psychotic disorder or other psychiatric morbidity. We studied the potential association between serum S100B and neuroimaging markers in patients with psychotic disorder, their non-psychotic siblings and controls. Our aim was to assess S100B-associated alterations in brain structure in the context of increased risk (patients and siblings) and disease-effects (patients) pertaining to psychotic disorder. In other words, we tested whether a potential association between $\mathrm{S} 100 \mathrm{~B}$ and brain measures was moderated by psychosis risk. 


\section{METHODS AND MATERIALS}

\section{Participants}

Data was collected in the context of a longitudinal study in the Netherlands (Habets et al., 2011; Korver et al., 2012), specifically in Maastricht and surroundings (including representative parts of Belgium). Patients with a minimum age of 16 years with a diagnosis of non-affective psychosis were included. They were recruited through the mental health services where they were treated, either as outpatients (majority of the sample) or inpatients. Diagnosis was based on DSM-IV criteria (APA, 2000), assessed with the Comprehensive Assessment of Symptoms and History (CASH) interview (Andreasen et al., 1992). Siblings were sampled through participating patients. Control subjects were recruited from the same area as described above, using random mailings in nearby municipalities and through advertisement in newspapers. The CASH was also used to confirm the absence of a diagnosis of non-affective psychosis in the siblings, and absence of lifetime diagnosis of psychotic disorder in the control subjects. For the control subjects, the occurrence of any psychotic disorder in either the subject or any first-degree family member, assessed using the Family Interview for Genetic Studies, constituted an exclusion criterion. Sufficient command of the Dutch language was an additional criterion for inclusion.

Additional exclusion criteria constituted oncologic processes, autoimmune disease, current infectious disease, cardiovascular disease and neurological disease.

The cohort consisted of 32 patients with a psychotic disorder, 44 siblings and 26 controls. Of note, the present study population is a subsample of the Maastricht cohort from our original S100B study (van der Leeuw et al., 2013). The subsample comprised all participants of whom both blood serum and magnetic resonance imaging (MRI) scans were available. Therefore, this sample is a random result of logistics, i.e. no specific selection was made from the original sample based on demographic characteristics.

Of the patients, 21 were diagnosed with schizophrenia and 9 were diagnosed with schizoaffective disorder. One patient was diagnosed with psychotic disorder not otherwise specified (NOS), and one patient had a diagnosis of substance-induced psychotic disorder. As non-psychotic psychiatric morbidity was not an exclusion criterion for siblings and controls, psychiatric diagnoses were present in these groups. The sample thus included 17 siblings and 5 controls with a history of major depressive disorder (MDD).

A total of 14 families participated in the study. Nine families contributed one patient and one sibling and one family contributed one patient and two siblings. Three families contributed two siblings, but no patients. One family contributed three siblings, but no patients. Twenty-two independent patients, 24 independent siblings and 26 independent controls participated, i.e. these individuals had no family ties within the sample. 


\section{Blood sample acquisition and processing}

Blood serum samples were acquired by venipuncture and were centrifuged within 24 hours and frozen until analysis. S100B was analyzed using a Liaison automated chemiluminescence analyzer according to the manufacturer's instructions (Diasorin). The lowest concentration of detection was 0.02 microgram per liter. Receiver operator curves showed best accuracy for the Liaison Sangtec 100 assay (Smit et al., 2005).

\section{MRI acquisition and processing}

MRI scans were obtained at Maastricht University, the Netherlands, using an Allegra syngo MR A30 (Siemens, Erlangen, Germany) operating at 3.0 Tesla. The following anatomical scan parameters were used: Modified Driven Equilibrium Fourier Transform (MDEFT) sequence; 176 slices, $1 \mathrm{~mm}$ isotropic voxel size, echo time $2.4 \mathrm{msec}$, repetition time $7.92 \mathrm{msec}$, inversion time $910 \mathrm{msec}$, flip angle 15 acquisition time 12 min $51 \mathrm{sec}$; Magnetization Prepared Rapid Acquisition Gradient-Echo (MPRAGE; Alzheimer's Disease Neuroimaging Initiative (ADNI)) sequence 192 slices, $1 \mathrm{~mm}$ isotropic voxel size, echo time $2.6 \mathrm{msec}$, repetition time $2250 \mathrm{msec}$, inversion time $900 \mathrm{msec}$, flip angle $9^{\circ}$, total acquisition time $7 \mathrm{~min} 23 \mathrm{sec}$. The matrix size was $256 \times 256$ and field of view was $256 \times 256 \mathrm{~mm}^{2}$. The number of excitations was one. Due to a scanner update during data collection nine participants (3 patients, 5 siblings and one control) underwent the MDEFT sequence, while for the majority the ADNI sequence was used.

Microstructural white matter anatomy was examined using diffusion tensor imaging (DTI) with an echo-planar-imaging sequence (field of view $230 \times 230$ mm², TR 10800 ms, TE 84 ms, voxel size 1.8 $\times 1.8 \times 1.8 \mathrm{~mm}^{3}$, b-value $1000 \mathrm{~s} / \mathrm{mm}^{2}$, noise level 40, 85 slices, no overlap). As a result of the scanner update, two DTI sequences were used: one with 76 directions (of which 4 T2-weighted (B0) and 72 diffusion-weighted (B)) and one with 81 directions ( $8 \times \mathrm{B} 0$ and 73xB). Only ten participants (3 patients, 6 siblings and 1 control) underwent the sequence with 76 directions. Total acquisition time of the DTI sequence was 15 minutes. Two male siblings did not complete the DTI sequence and were excluded from the analysis.

\section{Cortical thickness (CT) measurement}

Scans were processed and analyzed using Freesurfer stable release v5.3.0, http://surfer.nmr.mgh. harvard.edu (Dale et al., 1999; Fischl and Dale, 2000; Fischl et al., 2002; Fischl et al., 1999; Segonne et al., 2004). To measure $C T$, the cerebral cortex was parcellated into units based on gyral and sulcal structure (Desikan et al., 2006; Fischl et al., 2004). Furthermore, a variety of surface-based data was created including maps of curvature and sulcal depth. This method used both intensity and continuity information from the entire three dimensional MR volume in segmentation and deformation procedures to produce representations of $\mathrm{CT}$, calculated as the closest distance from the grey/white matter boundary to the grey matter/CSF boundary at each vertex on the tessellated surface (Fischl 
and Dale, 2000). The maps were created using spatial intensity gradients across tissue classes and were not restricted to the voxel resolution of the original data, thus were capable of detecting submillimeter differences between groups. CT measurement procedures have been validated against histological analysis (Rosas et al., 2002) and manual measurements (Kuperberg et al., 2003; Salat et al., 2004). Individual CT values for each predefined region of interest (hereafter: ROl; adapted from the Desikan atlas (Desikan et al., 2006), 34 ROls per hemisphere) were derived by FreeSurfer and exported to STATA version 13 (StataCorp). Thus, every individual had 68 CT measurements over the predefined ROls.

\section{Fractional anisotropy (FA) measurement}

Processing of DTI data was effectuated using tract-based spatial statistics (TBSS) v1.2 in FSL 4.1.6 (FMRIB Analysis Group, Oxford, UK, http://www.fmrib.ox.ac.uk/analysis/research/tbss). First, standard Siemens DICOM files were transformed into NIFTI format using a custom built in-house software package named GIANT (General Image ANalysis Tools developed by EHBMG). Raw data were corrected for head movement and eddy currents invoked during scanning. The BO volume was skull-stripped using FSL's Brain Extraction Tool (Smith, 2002) and this served as a brain mask for all B volumes. The next step was fitting a diffusion tensor model at each voxel using data output from the brain extraction, diffusion weighted data and gradient directions following a general linear model (FreeSurfer v5.3.0). After tensor fitting the process continued working on FA volumes, eroding them slightly.

Nonlinear registration aligned each FA volume to $1 \times 1 \times 1 \mathrm{~mm}$ standard FMRIB58_FA space. The standard FMRIB58_FA contains a template derived from high-resolution images of 58 participants in a well-aligned population (both males and females ranging between 20 and 50 years of age) (Smith et al., 2006). After nonlinear transformation of the FA volumes into standard space, a mean FA skeleton from all participants per group was derived. The mean FA skeleton follows the major white matter tracts in each individual participant (normalized in MNI152 space) and provides a way to compare between (groups of) participants. The final step of the processing was setting the FA threshold using visual inspection of the FA skeleton, in the present study at a level of 0.25 , to include major white matter tracts whilst removing small peripheral tracts that would cause excess interparticipant variability. In addition, this threshold setting avoided inclusion of regions that are likely to be composed of multiple tissue types or fiber orientations.

The Johns Hopkins University International Consortium for Brain Mapping (JHU ICBM)-DTI-81 white matter atlas labels (Mori et al., 2008) were used to assign specific tract names. From all $38 \mathrm{JHU}$ labeled white matter tracts, mean FA values were extracted and exported to STATA version 13 (StataCorp).

The study was approved by the standing ethics committee. All subjects provided written informed consent in accordance with the committee's guidelines. 


\section{Statistical analyses}

Conform previous work in this sample (Habets et al., 2011), datasets were transformed from a wide to long format resulting in a hierarchical structure, with 68 regional CT and 38 DTI measures (level 1) nested in respectively 102 and 100 participants (level 2). Given the clustering of brain measures within participants, and participants within families, multilevel random regression models were fitted (Goldstein, 1987) using the XTMIXED command in STATA (StataCorp, version 13), to account for compromised statistical independence of the observations.

Group $\times$ S100B interaction terms were evaluated using the Wald test (Clayton and Hills, 1993). A priori planned stratified analyses per group were conducted using the STATA margin command. In order to increase power, sensitivity analyses were conducted in healthy individuals (combining controls and siblings) and individuals with (increased risk of) psychotic disorder (combining siblings and patients). All analyses were adjusted for age, body mass index (BMI), scan sequence and sex.

\section{RESULTS}

\section{Descriptive analyses}

There was an uneven sex distribution in the patient group, with fewer females (28\%) than males. Controls were slightly older than siblings and patients, although not significantly so (Table 1). Patients $(P)$ had a higher mean BMI than controls $(C)$ and siblings $(S)$, the latter at trend-level ( $P$ vs. $C: B=2.331$, $95 \% \mathrm{Cl} 0.188$ to $4.475, \mathrm{p}=0.033$; $\mathrm{P}$ vs. S: $1.835,95 \% \mathrm{Cl}-0.051$ to $3.720, p=0.056$ ), whereas siblings and controls did not differ significantly ( $\mathrm{S}$ vs. C: $\mathrm{B}=0.497,95 \% \mathrm{Cl}-1.511$ to $2.505, \mathrm{p}=0.625$ ).

Table 1. Demographic characteristics

\begin{tabular}{llll}
\hline & Controls & Siblings & Patients \\
\hline Number of participants & 26 & 44 & 32 \\
Sex $($ male / female) & $11 / 15$ & $24 / 20$ & $25 / 7$ \\
S100B $(\mu \mathrm{g} / \mathrm{l})$ & $0.083 \pm 0.031$ & $0.087 \pm 0.044$ & $0.067 \pm 0.029$ \\
CT & $2.488 \pm 0.086$ & $2.499 \pm 0.092$ & $2.446 \pm 0.113$ \\
FA & $0.588 \pm 0.081$ & $0.575 \pm 0.082$ & $0.572 \pm 0.085$ \\
Age & $33.77 \pm 11.16$ & $30.39 \pm 7.96$ & $30.78 \pm 6.49$ \\
BMI & $23.65 \pm 3.36$ & $24.15 \pm 4.38$ & $25.99 \pm 4.21$ \\
Present use of AP (yes / no) & & & $27 / 5$ \\
Type of AP (FGA / SGA \& TGA) & & & $2 / 25$ \\
\hline
\end{tabular}

Means \pm standard deviations reported.

FGA: first generation antipsychotic medication (AP); SGA: second generation AP; TGA: third generation AP. 
Twenty-seven out of 32 patients reported current use of antipsychotic (AP) medication. Nine patients used risperidone, four used olanzapine, three used aripiprazole, two used clozapine, two quetiapine and two used amisulpride. There was a single case of bromperidol use. One person used a combination of risperidone and paliperidone, one used clozapine and aripiprazole, one person used aripiprazole and olanzapine, and one person used haloperidol and pipamperone.

\section{Mean S100B, CT and FA}

Mean S100B levels appeared lower in patients than in controls and siblings, with siblings having the highest values (Table 1). Regression analyses corrected for potential confounders (sex, age and BMI) showed that there was no significant difference in S100B serum levels between patients and controls ( $P$ vs. $C: B=-0.013,95 \% \mathrm{Cl}-0.033$ to $0.007, p=0.208$ ), in line with our first $\mathrm{S100B}$ study (van der Leeuw et al., 2013). However, patients did have lower S100B levels than siblings ( $P$ vs. S: $B=-0.019,95 \% C l$ -0.036 to $-0.002, p=0.031)$. There was no significant difference between $\mathrm{S} 100 \mathrm{~B}$ in siblings and controls ( $B=0.006,95 \% \mathrm{Cl}-0.012$ to $0.024, \mathrm{p}=0.529$ ).

Mean CT was significantly lower in patients than in controls and siblings when corrected for sex, age, scan sequence, handedness and highest level of completed education ( $P$ vs. C: $B=-0.051,95 \%$ $\mathrm{Cl}-0.096$ to $-0.006, \mathrm{p}=0.027$; $\mathrm{P}$ vs. $\mathrm{S}: \mathrm{B}=-0.042,95 \% \mathrm{Cl}-0.082$ to $-0.003, \mathrm{p}=0.034)$, with no significant difference between siblings and controls $(B=-0.009,95 \% \mathrm{Cl}-0.048$ to $0.031, p=0.670)$.

Mean FA was significantly lower in patients and siblings compared to controls, when controlled for sex, age, scan sequence, handedness and highest level of completed education ( $P$ vs. $C$ : $B=-0.020$, $95 \% \mathrm{Cl}-0.030$ to $-0.010, p=0.000 ; \mathrm{S}$ vs. $\mathrm{C}: \mathrm{B}=-0.015,95 \% \mathrm{Cl}-0.024$ to $-0.005, p=0.002 ; \mathrm{P}$ vs. $\mathrm{S}: \mathrm{B}=-0.005$, $95 \% \mathrm{Cl}-0.014$ to $0.003, p=0.233)$.

\section{Associations between S100B and brain structure}

No significant associations between S100B and $C T(B=0.378,95 \% C l-0.068$ to $0.825, p=0.097)$ and $\mathrm{S} 100 \mathrm{~B}$ and $\mathrm{FA}(\mathrm{B}=-0.006,95 \% \mathrm{Cl}-0.105$ to $0.094, \mathrm{p}=0.908)$ were observed in the total sample.

\section{Interactions}

Neither the group $\times$ S100B in the CT model $\left(x^{2}=0.59, p=0.743\right)$, nor the group $\times$ S100B interaction in the FA model ( $\left.x^{2}=3.72, p=0.156\right)$ were significant, confirmed by stratified analyses (Table 2 ).

Table 2. Associations between S100B and cerebral structural measures

\begin{tabular}{llll}
\hline & Controls & Siblings & Patients \\
\cline { 2 - 4 } & B, p-value & B, p-value & B, p-value \\
\hline Cortical thickness & $0.101(0.850)$ & $0.434(0.135)$ & $0.045(0.930)$ \\
Fractional anisotropy & $-0.148(0.192)$ & $-0.030(0.621)$ & $0.143(0.173)$ \\
\hline
\end{tabular}

B's and p-values reported. B represents the regression coefficient of the multilevel regression analyses. NA: not available. 


\section{Sensitivity analyses}

Combining controls and siblings into one "healthy" group $(n=70)$ showed no associations between S100B and brain measures (CT: $B=0.318,95 \% \mathrm{Cl}-0.108$ to $0.745, p=0.144$; FA:

$\mathrm{B}=-0.079,95 \% \mathrm{Cl}-0.189$ to $0.031, \mathrm{p}=0.161)$. Nor did the combination of siblings and patients into an (at risk of) psychosis group ( $\mathrm{n}=76$ ) result in significant findings (CT: $\mathrm{B}=0.474,95 \% \mathrm{Cl}-0.042$ to 0.991 , $p=0.072 ; F A: B=0.029,95 \% C l-0.073$ to $0.131, p=0.575)$.

\section{DISCUSSION}

We investigated the potential relationship between serum S100B and structural cerebral measures in both healthy subjects (in replication of previous work) and individuals with (increased risk of) psychotic disorder (not examined previously). No significant associations between serum S100B and cerebral structural measures were found in health or in the presence of (increased risk of) psychotic disorder.

\section{Findings}

Our first S100B study showed no association between (risk of) psychotic disorder and S100B (van der Leeuw et al., 2013). We now report no association between S100B and brain structure in the presence of (increased risk of) psychotic disorder in the same sample. Unfortunately these results cannot be surveyed in the broader context of schizophrenia research as there are no analogous studies (yet) to compare it with.

Furthermore, we do not find that CT and FA are consistently associated with S100B in controls and healthy siblings. The only previous study to explore the association between S100B and brain structure using neuroimaging was performed in healthy controls (Streitburger et al., 2012). This study suggested S100B-related demyelination in women (negative correlation with FA and a positive correlation with radial diffusivity). The analyses conducted by Streitbürger and colleagues were a priori stratified per sex, although they proceed to conclude that true sex-related differential expression patterns of S100B in the brain were not anticipated. Indeed, we did not explore sex differences in the present study as our original study did not show significant group $\times$ sex interactions in two large independent samples (van der Leeuw et al., 2013).

We did not find any significant associations between S100B and grey matter status represented by cortical thickness. This is in line with Streitbürger's finding of absence of association between S100B and grey matter density (Streitburger et al., 2012).

Due to the absence of association between serum S100B and brain structure in the current study's patient group, we are unable to make any deductions about S100B's cerebral actions in schizophrenia. S100B has dual effects: at physiological intracellular levels and nanomolar extracellular concentrations it is neurotrophic (Donato et al., 2013); at micromolar levels it becomes neurotoxic (Donato et al., 2013; Sen and Belli, 2007; van Beveren et al., 2006). As Streitbürger (Streitburger et al., 2012) and Schmitt 
(Schmitt et al., 2005) have pointed out, S100B elevation in schizophrenia is within the nanomolar range. That is to say that the micromolar S100B concentrations seen in clear neuronal damage (e.g. after traumatic brain injury or infarction) do not seem applicable to schizophrenia. S100B elevation in the nanomolar range is compatible with neurotrophic action. This makes a neurodegenerative process improbable and regenerative action more likely (Schmitt et al., 2005; Streitburger et al., 2012). The interpretation of our results may be complicated by the fact that mean S100B was lowest in patients, significantly so compared to siblings. Noteworthy is the fact that mean S100B was highest in the patient groups in the original study (van der Leeuw et al., 2013), although there were no significant differences in S100B levels between groups in the analyses. As the present sample (a subsample from the original study) is a result of logistics and not of specific selection criteria, we have no explanation for the low S100B levels in the present patient group and therefore conclude that these likely represent chance.

\section{Methodological considerations}

There are several limitations in the present study that deserve discussion.

First, scanner updates took place at an early stage during the data collection period. This led to an uneven distribution of scan types (within and between groups). The majority of participants underwent the ADNI sequence and DTI acquisition with 81 directions, though 9 MDEFT sequence scans and 10 DTI scans with 76 directions were obtained. To account for potential confounding by uneven distribution over the groups, scan type was included as a covariate in all analyses. As an extra test, we repeated analyses excluding the MDEFT sequence and the DTI scans with 76 directions. This did not alter the associations between S100B and CT and FA respectively (results not shown).

Second, there were 17 siblings and 5 controls with a history of MDD in this study sample. The findings from our original study indicated that there were no differences in serum S100B between these groups and that the inclusion of MDD and other psychiatric comorbidity in these groups did not alter the pattern of negative findings. As the original study (van der Leeuw et al., 2013) consisted of two independent samples of ample size (allowing for independent replication of our findings) we presume that the findings in controls and siblings were not influenced by MDD. We also base the combination of controls and siblings into a "healthy" group on the premise of absence of associations between S100B and group in the original study.

Third, mean CT and FA are coarse measures of brain structure. Taking this into consideration, in combination with small sample size, the present study may best be considered as hypothesis-generating. As this is the first study to investigate an association between S100B and cerebral structural measures in psychotic disorder, no comparison can be made to previous work. Naturally, replication of our study in a larger cohort is required to either corroborate or refute findings.

\section{Conclusions}

No evidence was present for S100B as a proxy marker of grey or white matter status. The association between S100B and brain measures was not moderated by psychosis risk. 


\section{REFERENCES}

Aleksovska, K., Leoncini, E., Bonassi, S., Cesario, A., Boccia, S., Frustaci, A., 2014. Systematic Review and Meta-Analysis of Circulating S100B Blood Levels in Schizophrenia. PLoS One 9(9), e106342.

Andreasen, N.C., Flaum, M., Arndt, S., 1992. The Comprehensive Assessment of Symptoms and History (CASH). An instrument for assessing diagnosis and psychopathology. Arch Gen Psychiatry 49(8), 615-623.

Buschert, J., Hohoff, C., Touma, C., Palme, R., Rothermundt, M., Arolt, V., Zhang, W., Ambree, O., 2013. S100B overexpression increases behavioral and neural plasticity in response to the social environment during adolescence. J Psychiatr Res 47(11), 1791-1799.

Clayton, D., Hills, M., 1993. Statistical Models in Epidemiology. Oxford University Press, Oxford.

Dale, A.M., Fischl, B., Sereno, M.I., 1999. Cortical surface-based analysis. I. Segmentation and surface reconstruction. Neuroimage 9(2), 179-194.

Desikan, R.S., Segonne, F., Fischl, B., Quinn, B.T., Dickerson, B.C., Blacker, D., Buckner, R.L., Dale, A.M., Maguire, R.P., Hyman, B.T., Albert, M.S., Killiany, R.J., 2006. An automated labeling system for subdividing the human cerebral cortex on MRI scans into gyral based regions of interest. Neuroimage 31(3), 968-980.

Donato, R., Cannon, B.R., Sorci, G., Riuzzi, F., Hsu, K., Weber, D.J., Geczy, C.L., 2013. Functions of S100 proteins. Curr Mol Med 13(1), 24-57.

Fischl, B., Dale, A.M., 2000. Measuring the thickness of the human cerebral cortex from magnetic resonance images. Proc Natl Acad Sci U S A 97(20), 11050-11055.

Fischl, B., Salat, D.H., Busa, E., Albert, M., Dieterich, M., Haselgrove, C., van der Kouwe, A., Killiany, R., Kennedy, D., Klaveness, S., Montillo, A., Makris, N., Rosen, B., Dale, A.M., 2002. Whole brain segmentation: automated labeling of neuroanatomical structures in the human brain. Neuron 33(3), 341-355.

Fischl, B., Sereno, M.I., Dale, A.M., 1999. Cortical surface-based analysis. II: Inflation, flattening, and a surface-based coordinate system. Neuroimage 9(2), 195-207.

Fischl, B., van der Kouwe, A., Destrieux, C., Halgren, E., Segonne, F., Salat, D.H., Busa, E., Seidman, L.J., Goldstein, J., Kennedy, D., Caviness, V., Makris, N., Rosen, B., Dale, A.M., 2004. Automatically parcellating the human cerebral cortex. Cereb Cortex 14(1), 11-22.

Goldstein, H., 1987. Multilevel Models in Educational and Social Research. Griffin, London.

Habets, P., Marcelis, M., Gronenschild, E., Drukker, M., van Os, J., 2011. Reduced cortical thickness as an outcome of differential sensitivity to environmental risks in schizophrenia. Biological psychiatry 69(5), 487-494.

Kleindienst, A., Harvey, H.B., Rice, A.C., Muller, C., Hamm, R.J., Gaab, M.R., Bullock, M.R., 2004. Intraventricular infusion of the neurotrophic protein $\mathrm{S100B}$ improves cognitive recovery after fluid percussion injury in the rat. Journal of neurotrauma 21(5), 541-547.

Kleindienst, A., McGinn, M.J., Harvey, H.B., Colello, R.J., Hamm, R.J., Bullock, M.R., 2005. Enhanced hippocampal neurogenesis by intraventricular $\mathrm{S100B}$ infusion is associated with improved cognitive recovery after traumatic brain injury. Journal of neurotrauma 22(6), 645-655.

Korver, N., Quee, P.J., Boos, H.B., Simons, C.J., de Haan, L., 2012. Genetic Risk and Outcome of Psychosis (GROUP), a multi site longitudinal cohort study focused on gene-environment interaction: objectives, sample characteristics, recruitment and assessment methods. Int J Methods Psychiatr Res.

Kuperberg, G.R., Broome, M.R., McGuire, P.K., David, A.S., Eddy, M., Ozawa, F., Goff, D., West, W.C., Williams, S.C., van der Kouwe, A.J., Salat, D.H., Dale, A.M., Fischl, B., 2003. Regionally localized thinning of the cerebral cortex in schizophrenia. Arch Gen Psychiatry 60(9), 878-888.

Mori, S., Oishi, K., Jiang, H., Jiang, L., Li, X., Akhter, K., Hua, K., Faria, A.V., Mahmood, A., Woods, R., Toga, A.W., Pike, G.B., Neto, P.R., Evans, A., Zhang, J., Huang, H., Miller, M.I., van Zijl, P., Mazziotta, J., 2008. Stereotaxic white matter atlas based on diffusion tensor imaging in an ICBM template. Neurolmage 40(2), 570-582. 
Rosas, H.D., Liu, A.K., Hersch, S., Glessner, M., Ferrante, R.J., Salat, D.H., van der Kouwe, A., Jenkins, B.G., Dale, A.M., Fischl, B., 2002. Regional and progressive thinning of the cortical ribbon in Huntington's disease. Neurology 58(5), 695-701.

Salat, D.H., Buckner, R.L., Snyder, A.Z., Greve, D.N., Desikan, R.S., Busa, E., Morris, J.C., Dale, A.M., Fischl, B., 2004. Thinning of the cerebral cortex in aging. Cereb Cortex 14(7), 721-730.

Schmitt, A., Bertsch, T., Henning, U., Tost, H., Klimke, A., Henn, F.A., Falkai, P., 2005. Increased serum S100B in elderly, chronic schizophrenic patients: negative correlation with deficit symptoms. Schizophr Res 80(2-3), 305-313.

Segonne, F., Dale, A.M., Busa, E., Glessner, M., Salat, D., Hahn, H.K., Fischl, B., 2004. A hybrid approach to the skull stripping problem in MRI. Neuroimage 22(3), 1060-1075.

Sen, J., Belli, A., 2007. S100B in neuropathologic states: the CRP of the brain? J Neurosci Res 85(7), 1373-1380.

Smit, L.H., Korse, C.M., Bonfrer, J.M., 2005. Comparison of four different assays for determination of serum S-100B. Int J Biol Markers 20(1), 34-42.

Smith, S.M., 2002. Fast robust automated brain extraction. Human brain mapping 17(3), 143-155.

Smith, S.M., Jenkinson, M., Johansen-Berg, H., Rueckert, D., Nichols, T.E., Mackay, C.E., Watkins, K.E., Ciccarelli, O., Cader, M.Z., Matthews, P.M., Behrens, T.E., 2006. Tract-based spatial statistics: voxelwise analysis of multi-subject diffusion data. Neurolmage 31(4), 1487-1505.

Streitburger, D.P., Arelin, K., Kratzsch, J., Thiery, J., Steiner, J., Villringer, A., Mueller, K., Schroeter, M.L., 2012. Validating Serum S100B and Neuron-Specific Enolase as Biomarkers for the Human Brain - A Combined Serum, Gene Expression and MRI Study. PLoS One 7(8), e43284.

van Beveren, N.J., van der Spelt, J.J., de Haan, L., Fekkes, D., 2006. Schizophrenia-associated neural growth factors in peripheral blood. A review. Eur Neuropsychopharmacol 16(7), 469-480.

van der Leeuw, C., Marcelis, M., Peeters, S.C., Verbeek, M.M., Menheere, P.P., de Haan, L., van Os, J., van Beveren, N.J., 2013. Replicated Evidence of Absence of Association between Serum S100B and (Risk of) Psychotic Disorder. PLoS One 8(12), e82535. 



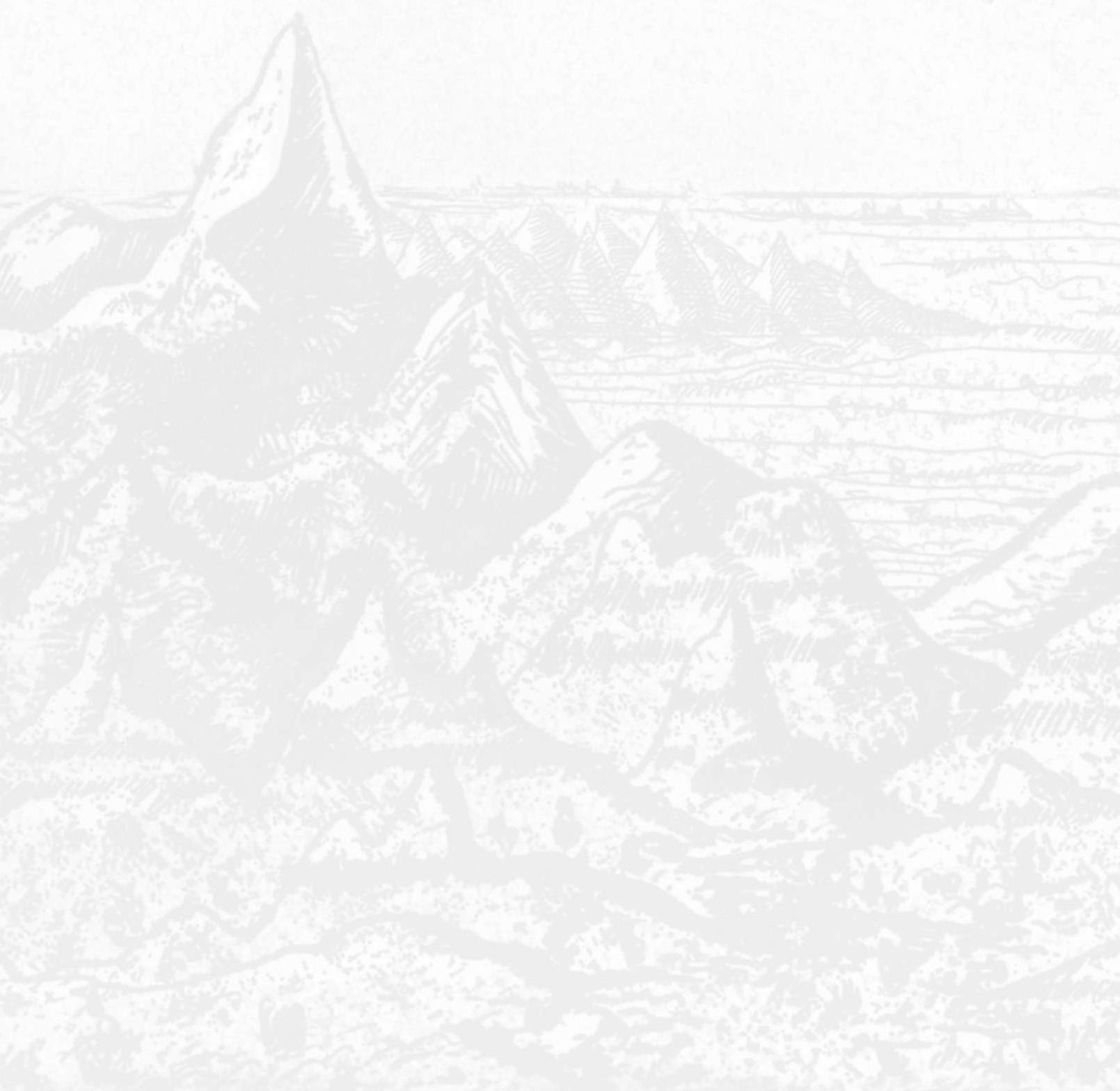


Seven

General discussion 

The studies described in this thesis examined potential biological endophenotypes in relation to structural cerebral measures in psychotic disorder. Bone mineral density (BMD) and the protein S100B were investigated. To validate these peripheral markers as indicators of cerebral status, associations with indices of cerebral structure (i.e. grey and white matter status represented by cortical thickness (CT) and fractional anisotropy (FA) respectively) were assessed.

This discussion aims to integrate findings and view them in the broader context of present concepts applied in psychiatric research.

\section{Bone mineral density}

Altered estrogen-induced neuroprotection (reflected by reduced BMD) may be an etiological factor in schizophrenia (Maric et al., 2005). We found that reduced femoral BMD was associated with being female and having a psychotic disorder; and that BMD reduction could be partially, but not fully, ascribed to the use of prolactin (PRL)-raising antipsychotics (AP) (chapter 2). Environmental factors, in addition to primary low estrogen levels in women, may impact the risk of developing psychosis (van der Leeuw et al., 2013a). In line with our findings, women with schizophrenia have previously been identified as at risk of reduced BMD (Meaney and O'Keane, 2007) and schizophrenia has been proposed as an independent determinant of poor skeletal status in women, after adjusting for AP use (among other disease-related factors) (Partti et al., 2010).

BMD was not reduced in unaffected siblings of patients with psychotic disorder, and therefore this data suggests it is not an endophenotypic marker of psychotic disorder (chapter 2). BMD had not previously been examined as an endophenotype.

To our knowledge, the study described in chapter 3 is the first to examine associations between cumulative estrogen exposure (to the extent that it is reflected by femoral BMD) and brain structure in psychotic disorder. Our findings suggest that higher cumulative exposure to estrogen is associated with increased cerebral cortical thickness in women with psychotic disorder. There was no evidence of association between lifetime estrogen exposure and white matter integrity (van der Leeuw et al., 2013b). Interpretation of these findings is complicated by the cross-sectional nature of the data, as well as the fact that no previous studies have been conducted combining (markers) of estrogen exposure and structural brain alterations in psychiatrically ill individuals. Nevertheless it is biologically plausible that estrogen, through its impact on brain morphology, contributes to the risk of - and sex differences - in psychotic disorder.

A major distinction of the BMD studies in this thesis is that they are unique in their focus on a possible etiological mechanism, i.e. primary low estrogen levels. The findings of the first two studies (chapters 2 and 3) support both aspects (deficiency and protection) of the estrogen hypothesis (Riecher-Rossler, 2002; Riecher-Rossler and Hafner, 1993). Low BMD is possibly indicative of estrogen deficiency and BMD is positively associated with CT (neuroprotection).

There was no evidence of ongoing estrogen deficiency in psychotic disorder as there was no excessive bone loss over a 3-year period in patients using AP (chapter 4). In effect, femoral BMD measures 
decreased significantly less in patients compared to controls. As age was the only moderator of bone mass loss that survives Bonferroni correction in a recent meta-analysis (Stubbs et al., 2014), our patients were possibly too young at 11 to show disproportionate BMD loss compared to older controls (3 years senior to patients at a mean age of 35). Furthermore, in line with the results of the study described in chapter 2, siblings did not differ from controls at follow-up in the cross-sectional analysis nor did they show accelerated bone loss. This confirms that BMD does not appear to be an endophenotype of psychotic disorder.

Larger longitudinal studies are necessary. However, designing a study to elucidate a primary etiological effect for estrogen in psychotic disorder remains challenging. BMD is a proxy marker of lifetime estrogen exposure which is influenced by many (non-) hormonal factors, and is, at best, an indirect marker of estrogen-mediated neuroprotection. The absence of estrogen and prolactin levels is a caveat in our studies. Though given our primary interest in cumulative estrogen exposure, BMD was considered the best outcome measure. Also, interpretation of estrogen levels is complicated as its measurement is a momentary assessment of physiologically fluctuating concentrations. Notwithstanding, although estrogen and prolactin levels do not represent lifetime exposure, they would serve to confirm the presence of hypoestrogenism and hyperprolactinemia and may have contributed to the understanding of our findings.

\section{Effects of antipsychotic medication}

A recent review concluded that "schizophrenia is associated with reduced bone mineral density and fracture risk" (Kishimoto et al., 2012), possibly resulting from a high prevalence of risk factors for osteoporosis in this population (Halbreich, 2007). Another recent review reported that BMD is reduced in patients who use AP medication (Crews and Howes, 2012), with first generation and other prolactinraising APs being particularly implicated (Abraham et al., 2003; Bilici et al., 2002; Haddad and Wieck, 2004; Howard et al., 2007; Meaney and O'Keane, 2007; Meaney et al., 2004). Stubbs and colleagues conducted the first meta-analysis examining low bone mass in schizophrenia and concluded that patients with schizophrenia were more than twice as likely to have osteoporosis in comparison to age and sex matched controls (Stubbs et al., 2014).

Although the current use of a PRL-raising AP was found to be negatively associated with lumbar BMD in women at baseline (chapter 2), no conclusive proof of BMD alterations due to PRL-raising AP or other disease-related factors was found in the longitudinal data reported in chapter 4. Other longitudinal studies do find diminished BMD or altered bone metabolism due to AP-induced hyperprolactinemia. In total, four other studies have been carried out to date. Wang and colleagues found that hyperprolactinemia resulted in decreased total lumbar BMD in a group of male and female patients with schizophrenia who had been treated with PRL-raising AP for 12 months (Wang et al., 2014). An observational cohort study (Takahashi et al., 2013) examined longitudinal BMD changes in patients, comparing patients taking PRL-raising AP and PRL-sparing AP at first DEXA measurement. There was no significant BMD difference between these patient groups in any of the measurements, 
over a mean follow-up period of 3 years. However, a significant AP type by time interaction was found, possibly indicating a negative effect of prolactin-raising AP. Two studies examined AP effects on BMD specifically in women. Meaney and O'Keane (Meaney and O'Keane, 2007) followed premenopausal female patients with schizophrenia over the course of one year and compared lumbar and femoral BMD changes between those taking prolactin-sparing AP and those taking prolactin-raising AP. The efficacy of interventions (such as weight-bearing exercise, nutritional supplements and sex hormone replacement therapy) to ameliorate BMD was examined. Without intervention, women on a PRLraising $\mathrm{AP}$ showed a decrease in lumbar BMD compared to women taking a PRL-sparing AP. With intervention, patients using PRL-sparing AP $(n=4)$ showed a significant increase in lumbar BMD, while patients using a PRL-raising AP ( $n=12)$ also showed an increase, though not significantly so (Meaney and O'Keane, 2007). No differences in femoral BMD were found between the AP or intervention groups. Over a period of 12 months, Abraham et al. found that hyperprolactinemia accelerated bone metabolism, but this did not result in bone mass loss (Abraham et al., 2003). The follow-up duration of this study was possibly inadequate.

Optimistically, our findings may be reassuring for patients who take prolactin-raising AP. However, given the scarcity of longitudinal work and inconsistencies in outcome, it deserves recommendation to remain aware of sequelae of hyperprolactinemia such as hypogonadism and BMD loss, and to assess side-effects of prolactin-raising AP in each individual patient.

\section{S100B}

In two large independent groups, no statistically significant association between group and S100B was found. Serum S100B levels in patients and siblings were not different from controls (chapter 5). The absence of $\mathrm{S100B}$ elevation in siblings and relatively stable patients (with a mean illness duration of less than ten years) in our study, in combination with the previously reported positive findings in predominantly hospitalized patients (suggesting higher levels of symptomatology), suggest that S100B elevations may reflect active disease processes in certain patient populations, i.e. it is "state" dependent.

Other negative findings in S100B research are limited. One study found unaltered S100B levels in unmedicated patients compared to controls, though AP treatment was associated with S100B elevation (Schroeter et al., 2003). Another study showed S100B elevation in cerebrospinal fluid but not serum (Steiner et al., 2006). And there is one study that reported S100B reduction in schizophrenia (Gattaz et al., 2000).

Three meta-analyses have been carried out to date (Aleksovska et al., 2014; Schroeter et al., 2009; Schroeter and Steiner, 2009), all indicating S100B elevation in schizophrenia. The two publications by Schroeter are from the same year and the second meta-analysis includes one additional study to the first. The methodology reported in both publications is concise. The most recent systematic review and meta-analysis (Aleksovska et al., 2014), which included our study, is thorough and provides a detailed description of methodology. Twenty studies were included and critically appraised. Furthermore, 
funnel plots were examined and ruled out publication bias. This exhaustive study showed a significant increase in S100B in schizophrenia patients (meta mean ratio of 1.76, 95\% confidence interval 1.44 to $2.15, p<0.001)$. Stratified sensitivity analysis showed similar outcomes, regardless of medication status or disease stage. Although Aleksovska and colleagues carried out subgroup analyses heterogeneity remained high, warranting discretion in the interpretation of results.

To date, the validity of S100B as a proxy marker for grey and white matter status has not been investigated in patients with a psychotic disorder or other psychiatric morbidity. We studied the potential association between serum S100B and neuroimaging indices (CT and FA) in patients with psychotic disorder, their non-psychotic siblings and controls. Our aim was to assess S100B-associated altered brain structure in the context of increased risk (patients and siblings) and disease-effects (patients) of psychotic disorder.

There was no evidence that CT and FA are associated with S100B in controls and healthy siblings (chapter 6). The only previous study to explore the association between S100B and brain structure using neuroimaging was performed in healthy controls (Streitburger et al., 2012). This study suggested S100B-related demyelination in women. The analyses conducted by Streitburger and colleagues were a priori stratified per sex, although they proceed to conclude that true sex-related differential expression patterns of S100B in the brain were not anticipated (Streitburger et al., 2012). Indeed, there is no strong a priori hypothesis on S100B-related sex differences. Moreover, we did not explore sex differences in the present study as our original study did not show significant group $\times$ sex interactions in two large independent samples (van der Leeuw et al., 2013c).

We found no association between $\mathrm{S100B}$ and brain structure in the presence of psychotic disorder. Unfortunately these results cannot be surveyed in the broader context of schizophrenia research as there are no analogous studies (yet) to compare it with. Due to the absence of association between serum S100B and brain structure in the current study's patient group, we are unable to make any deductions about S100B's cerebral actions in schizophrenia.

S100B has dual effects: at physiological intracellular levels and nanomolar extracellular concentrations it is neurotrophic (Donato et al., 2013); at micromolar levels it becomes neurotoxic (Donato et al., 2013; Sen and Belli, 2007; van Beveren et al., 2006). As Streitbürger (Streitburger et al., 2012) and Schmitt (Schmitt et al., 2005) point out, S100B elevation in schizophrenia is within the nanomolar range. That is to say that the micromolar S100B concentrations seen in clear neuronal damage (e.g. after traumatic brain injury or infarction) do not seem applicable to schizophrenia. S100B elevation in the nanomolar range is compatible with neurotrophic action. This makes a neurodegenerative process improbable and if any S100B-action does occur, regeneration is more likely (Schmitt et al., 2005; Streitburger et al., 2012). 


\section{The endophenotype concept in psychiatry: how is it helpful?}

As Gottesman and Gould point out, "the brain is the most complex of all organs" (Gottesman and Gould, 2003). Unfortunately, the brain is also the organ with the least "access". Psychiatrists are unable to biopsy and examine the brains of their living patients under the microscope. This poses a complication unknown to other medical fields. Therefore, knowledge of endophenotypes is essential to be able to make deductions about the pathophysiology of psychiatric disorders and to further the development of treatment strategies. In addition, endophenotype studies can discriminate between etiological mechanisms and secondary effects of disease and treatment. Secondary disease effects may then receive adequate awareness and proper management.

For instance, our studies showed that bone mineral density and S100B elevation are not endophenotypes of psychotic disorder. In turn, we showed that estrogen deficiency, mirrored by reduced $B M D$, is a possible primary mechanism in the development of psychotic disorder in women. This provides an etiological basis for intervention studies that show beneficial effects of estrogen augmentation and the use of selective estrogen receptor modulators (SERMs) (Begemann et al., 2012; Kulkarni et al., 2014; Kulkarni et al., 2010) in women with schizophrenia. Theoretically, the effects of estrogen on both brain and bone are not specific to women: androgens are aromatized focally and reach biologically effective concentrations at these tissue sites in men (Boerma et al., 2010). Treatment studies in men (Kulkarni et al., 2011; Kulkarni et al., 2013) support this theory.

Furthermore, reduced BMD due to secondary disease factors (such as lifestyle and iatrogenic effects of PRL-raising AP) is an important issue. The use of prolactin-raising AP explained part of the variance in our cross-sectional study that found reduced femoral BMD in female patients (van der Leeuw et al., 2013a), but this was not confirmed in the longitudinal study. Vigilance of increased risk of osteoporosis and inherent fracture risk is warranted in the comprehensive treatment of all health issues in patients with psychotic disorder (Crews and Howes, 2012; Kishimoto et al., 2012; Stubbs et al., 2014).

\section{Biomarkers in psychiatry}

The term biological marker or biomarker refers to a characteristic that can be objectively measured and evaluated as an indicator of normal and pathogenic biological processes (van Beveren and Hoogendijk, 2011; van der Leeuw et al., 2013c). As is the case with endophenotypes, their identification may offer insight into etiological mechanisms of disease and lead to new treatment perspectives. In clinical practice, valid biomarkers assist in the diagnostic process and enable monitoring of disease course and treatment response (Allen et al., 2009; Pillai and Buckley, 2012; van Beveren and Hoogendijk, 2011). Biological markers may be primarily environmental, (patho) physiological or multifactorial. They can be state or trait markers. Unlike endophenotypes there is no necessary genetic underpinning. Currently, psychiatric diagnoses are based on clinical observation and course of symptoms. This can be an extended process that requires time, while time is an important determinant in prognosis. Fast and correct diagnosis is paramount. An etiological diagnosis would be superior, more reliable with less room for error, and may enable individual treatment and prediction of outcome (Lakhan et 
al., 2010; Singh and Rose, 2009). At present, no biomarkers have been identified that are specific to psychotic disorder or its prodromal stage (Bender et al., 2007).

$\mathrm{S} 100 \mathrm{~B}$ is a potential candidate marker. It has been coined the C-reactive protein (CRP) of the brain (Sen and Belli, 2007) as it is a potential indicator of neurological and psychiatric pathology from birth until elderly age (van der Leeuw et al., 2013c). From this description, one can make out that it is not a specific marker. S100B elevation is perhaps merely indicative of mental activity and/or stress. S100B elevation has been shown in physicians on duty, activity which may indeed be stressful but not quite pathological (Gazzolo et al., 2010). In schizophrenia, it appears to be predominantly a state marker of a psychotic phase rather than a trait marker. It remains unknown whether S100B is raised in the prodromal phase preceding the onset of frank psychosis (van Beveren et al., 2006).

The meta-analysis by Aleksovska and colleagues, indicating S100B elevation in schizophrenia, provides hope that useful markers can be identified (Aleksovska et al., 2014). As a single biomarker for psychotic disorder is highly unlikely to meet all the requirements described above, identification of a set of biomarkers for disorder subtypes, based on their underlying biological pathways, would be ideal (Sokolowska et al., 2013). Besides proteomics, (epi)genetics and neuroimaging can provide clinically useful biomarkers (Lakhan et al., 2010).

But how do we implement and utilize these techniques in clinical practice once their validity has been proven? Are we to draw blood from patients and subject them to lumbar punctures and MRIs during acute psychosis? These are patients who frequently don't allow physical examination and toxicology screening in urine, while these are non-invasive procedures. Informed consent for invasive diagnostic tests may very well not be possible in severe mental states. Do we then make use of sedation to be able to proceed with diagnostic assays? This would profoundly change our approach to acutely ill patients. Gaining their trust and building a constructive therapeutic relationship would not be prioritized initially. Ideally, we will eventually be able to identify vulnerable individuals during the prodromal phase, prior to the onset of frank psychosis.

Scientific advancement enables the better understanding of biological mechanisms in psychiatry. Optimistically, novel treatment options will not be far behind.

For now, many psychiatrists may feel they don't necessarily need a biological substrate to confirm or treat a clinical syndrome such as psychosis. This mentality will hold psychiatry back, lagging a hundred years behind other medical specialties. We should try to catch up. 


\section{REFERENCES}

Abraham, G., Paing, W.W., Kaminski, J., Joseph, A., Kohegyi, E., Josiassen, R.C., 2003. Effects of elevated serum prolactin on bone mineral density and bone metabolism in female patients with schizophrenia: a prospective study. Am J Psychiatry 160, 1618-1620.

Aleksovska, K., Leoncini, E., Bonassi, S., Cesario, A., Boccia, S., Frustaci, A., 2014. Systematic Review and Meta-Analysis of Circulating S100B Blood Levels in Schizophrenia. PLoS One 9, e106342.

Allen, A.J., Griss, M.E., Folley, B.S., Hawkins, K.A., Pearlson, G.D., 2009. Endophenotypes in schizophrenia: a selective review. Schizophrenia research 109, 24-37.

Begemann, M.J., Dekker, C.F., van Lunenburg, M., Sommer, I.E., 2012. Estrogen augmentation in schizophrenia: a quantitative review of current evidence. Schizophrenia research 141, 179-184.

Bender, S., Weisbrod, M., Resch, F., 2007. Which perspectives can endophenotypes and biological markers offer in the early recognition of schizophrenia? Journal of neural transmission 114, 1199-1215.

Bilici, M., Cakirbay, H., Guler, M., Tosun, M., Ulgen, M., Tan, U., 2002. Classical and atypical neuroleptics, and bone mineral density, in patients with schizophrenia. Int J Neurosci 112, 817-828.

Boerma, M.A., van der Stel, J.C., van Amelsvoort, T., Linszen, D.H., de Haan, L., 2010. [Women, schizophrenia and oestrogen; neurobiological hypotheses and hormonetherapy studies]. Tijdschrift voor psychiatrie 52, $235-244$.

Crews, M.P., Howes, O.D., 2012. Is antipsychotic treatment linked to low bone mineral density and osteoporosis? A review of the evidence and the clinical implications. Hum Psychopharmacol 27, 15-23.

Donato, R., Cannon, B.R., Sorci, G., Riuzzi, F., Hsu, K., Weber, D.J., Geczy, C.L., 2013. Functions of S100 proteins. Curr Mol Med 13, 24-57.

Gattaz, W.F., Lara, D.R., Elkis, H., Portela, L.V., Goncalves, C.A., Tort, A.B., Henna, J., Souza, D.O., 2000. Decreased S100beta protein in schizophrenia: preliminary evidence. Schizophr Res 43, 91-95.

Gazzolo, D., Florio, P., Zullino, E., Giovannini, L., Scopesi, F., Bellini, C., Peri, V., Mezzano, P., Petraglia, F., Michetti, F., 2010. $\mathrm{S} 100 \mathrm{~B}$ protein increases in human blood and urine during stressful activity. Clinical chemistry and laboratory medicine : CCLM / FESCC 48, 1363-1365.

Gottesman, II, Gould, T.D., 2003. The endophenotype concept in psychiatry: etymology and strategic intentions. The American journal of psychiatry 160, 636-645.

Haddad, P.M., Wieck, A., 2004. Antipsychotic-induced hyperprolactinaemia: mechanisms, clinical features and management. Drugs 64, 2291-2314.

Halbreich, U., 2007. Osteoporosis, schizophrenia and antipsychotics: the need for a comprehensive multifactorial evaluation. CNS Drugs 21, 641-657.

Howard, L., Kirkwood, G., Leese, M., 2007. Risk of hip fracture in patients with a history of schizophrenia. Br J Psychiatry 190, 129-134.

Kishimoto, T., De Hert, M., Carlson, H.E., Manu, P., Correll, C.U., 2012. Osteoporosis and fracture risk in people with schizophrenia. Curr Opin Psychiatry 25, 415-429.

Kulkarni, J., de Castella, A., Headey, B., Marston, N., Sinclair, K., Lee, S., Gurvich, C., Fitzgerald, P.B., Burger, H., 2011. Estrogens and men with schizophrenia: is there a case for adjunctive therapy? Schizophrenia research 125, 278-283.

Kulkarni, J., Gavrilidis, E., Wang, W., Worsley, R., Fitzgerald, P.B., Gurvich, C., Van Rheenen, T., Berk, M., Burger, H., 2014. Estradiol for treatment-resistant schizophrenia: a large-scale randomized-controlled trial in women of childbearing age. Molecular psychiatry.

Kulkarni, J., Gavrilidis, E., Worsley, R., Van Rheenen, T., Hayes, E., 2013. The role of estrogen in the treatment of men with schizophrenia. International journal of endocrinology and metabolism 11, 129-136.

Kulkarni, J., Gurvich, C., Lee, S.J., Gilbert, H., Gavrilidis, E., de Castella, A., Berk, M., Dodd, S., Fitzgerald, P.B., Davis, S.R., 2010. Piloting the effective therapeutic dose of adjunctive selective estrogen receptor modulator treatment in postmenopausal women with schizophrenia. Psychoneuroendocrinology 35, 1142-1147. 
Lakhan, S.E., Vieira, K., Hamlat, E., 2010. Biomarkers in psychiatry: drawbacks and potential for misuse. International archives of medicine 3,1

Maric, N., Popovic, V., Jasovic-Gasic, M., Pilipovic, N., van Os, J., 2005. Cumulative exposure to estrogen and psychosis: a peak bone mass, case-control study in first-episode psychosis. Schizophrenia research 73, 351-355.

Meaney, A.M., O'Keane, V., 2007. Bone mineral density changes over a year in young females with schizophrenia: relationship to medication and endocrine variables. Schizophrenia research 93, 136-143.

Meaney, A.M., Smith, S., Howes, O.D., O'Brien, M., Murray, R.M., O'Keane, V., 2004. Effects of long-term prolactinraising antipsychotic medication on bone mineral density in patients with schizophrenia. Br J Psychiatry 184, 503-508.

Partti, K., Heliovaara, M., Impivaara, O., Perala, J., Saarni, S.I., Lonnqvist, J., Suvisaari, J.M., 2010. Skeletal status in psychotic disorders: a population-based study. Psychosomatic medicine 72, 933-940.

Pillai, A., Buckley, P.F., 2012. Reliable biomarkers and predictors of schizophrenia and its treatment. Psychiatr Clin North Am 35, 645-659.

Riecher-Rossler, A., 2002. Oestrogen effects in schizophrenia and their potential therapeutic implications--review. Archives of women's mental health 5, 111-118.

Riecher-Rossler, A., Hafner, H., 1993. Schizophrenia and oestrogens--is there an association? European archives of psychiatry and clinical neuroscience 242, 323-328.

Schmitt, A., Bertsch, T., Henning, U., Tost, H., Klimke, A., Henn, F.A., Falkai, P., 2005. Increased serum S100B in elderly, chronic schizophrenic patients: negative correlation with deficit symptoms. Schizophr Res 80, 305-313.

Schroeter, M.L., Abdul-Khaliq, H., Fruhauf, S., Hohne, R., Schick, G., Diefenbacher, A., Blasig, I.E., 2003. Serum S100B is increased during early treatment with antipsychotics and in deficit schizophrenia. Schizophr Res 62, 231-236.

Schroeter, M.L., Abdul-Khaliq, H., Krebs, M., Diefenbacher, A., Blasig, I.E., 2009. Neuron-specific enolase is unaltered whereas S100B is elevated in serum of patients with schizophrenia--original research and meta-analysis. Psychiatry Res 167, 66-72.

Schroeter, M.L., Steiner, J., 2009. Elevated serum levels of the glial marker protein S100B are not specific for schizophrenia or mood disorders. Mol Psychiatry 14, 235-237.

Sen, J., Belli, A., 2007. S100B in neuropathologic states: the CRP of the brain? J Neurosci Res 85, 1373-1380.

Singh, l., Rose, N., 2009. Biomarkers in psychiatry. Nature 460, 202-207.

Sokolowska, I., Ngounou Wetie, A.G., Wormwood, K., Thome, J., Darie, C.C., Woods, A.G., 2013. The potential of biomarkers in psychiatry: focus on proteomics. Journal of neural transmission.

Steiner, J., Bielau, H., Bernstein, H.G., Bogerts, B., Wunderlich, M.T., 2006. Increased cerebrospinal fluid and serum levels of S100B in first-onset schizophrenia are not related to a degenerative release of glial fibrillar acidic protein, myelin basic protein and neurone-specific enolase from glia or neurones. J Neurol Neurosurg Psychiatry 77, 1284-1287

Streitburger, D.P., Arelin, K., Kratzsch, J., Thiery, J., Steiner, J., Villringer, A., Mueller, K., Schroeter, M.L., 2012. Validating Serum S100B and Neuron-Specific Enolase as Biomarkers for the Human Brain - A Combined Serum, Gene Expression and MRI Study. PLoS One 7, e43284.

Stubbs, B., De Hert, M., Sepehry, A.A., Correll, C.U., Mitchell, A.J., Soundy, A., Detraux, J., Vancampfort, D., 2014. A meta-analysis of prevalence estimates and moderators of low bone mass in people with schizophrenia. Acta psychiatrica Scandinavica 130, 470-486.

Takahashi, T., Uchida, H., John, M., Hirano, J., Watanabe, K., Mimura, M., Correll, C.U., Kishimoto, T., 2013. The impact of prolactin-raising antipsychotics on bone mineral density in patients with schizophrenia: findings from a longitudinal observational cohort. Schizophrenia research 147, 383-386.

van Beveren, N.J., Hoogendijk, W.J., 2011. Clinical utility of serum biomarkers for major psychiatric disorders. Int Rev Neurobiol 101, 351-374.

van Beveren, N.J., van der Spelt, J.J., de Haan, L., Fekkes, D., 2006. Schizophrenia-associated neural growth factors in peripheral blood. A review. Eur Neuropsychopharmacol 16, 469-480. 
van der Leeuw, C., Habets, P., Domen, P., van Kroonenburgh, M., van Os, J., Marcelis, M., 2013a. Bone mineral density as a marker of cumulative endogenous estrogen exposure: relationship to background genetic risk of psychotic disorder. Schizophrenia research 143, 25-31.

van der Leeuw, C., Habets, P., Gronenschild, E., Domen, P., Michielse, S., van Kroonenburgh, M., van Os, J., Marcelis, M., 2013b. Testing the estrogen hypothesis of schizophrenia: associations between cumulative estrogen exposure and cerebral structural measures. Schizophrenia research 150, 114-120.

van der Leeuw, C., Marcelis, M., Peeters, S.C., Verbeek, M.M., Menheere, P.P., de Haan, L., van Os, J., van Beveren, N.J., 2013c. Replicated Evidence of Absence of Association between Serum S100B and (Risk of) Psychotic Disorder. PLoS One 8, e82535.

Wang, M., Hou, R., Jian, J., Mi, G., Qiu, H., Cao, B., Tang, M., 2014. Effects of antipsychotics on bone mineral density and prolactin levels in patients with schizophrenia: a 12-month prospective study. Human psychopharmacology 29, 183-189. 


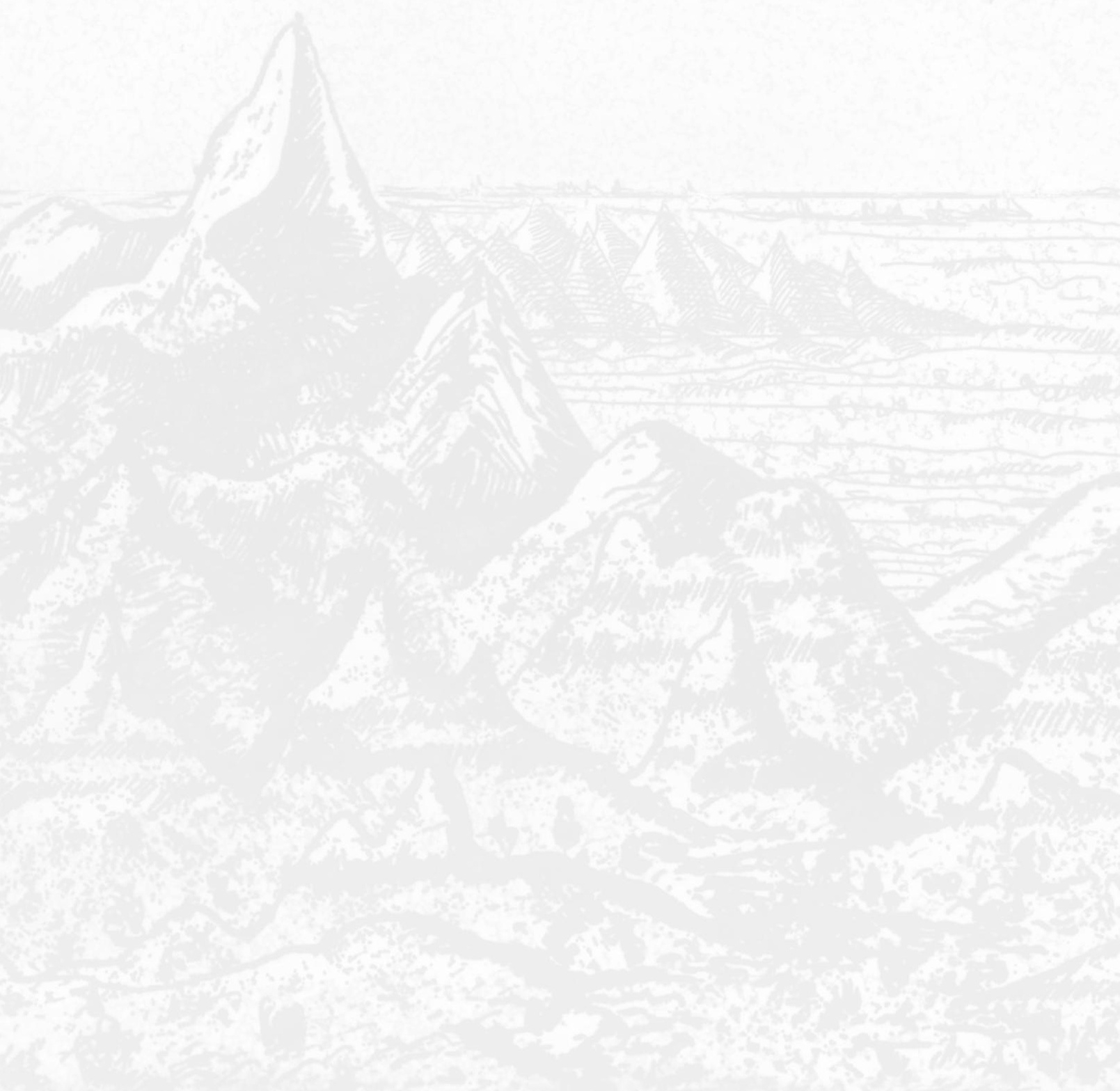


Eight

Summary 

Psychotic disorders, of which schizophrenia is perhaps the most prominent, are characterized by the presence of altered sensory perception (hallucinations) and/or disturbed thought content (delusions). Reality testing is inherently compromised. The lifetime prevalence rate of schizophrenia is said to be $1 \%$. The exact etiology of psychotic disorder is still unknown. Genetic and environmental risks interact and are thought to converge on a final common pathway to psychotic disorder, on which increased mesolimbic dopamine reactivity leads to hallucinations and delusions. Multiple biological mechanisms may influence or be influenced by dopaminergic function, while the role of other neurotranmitters should not be neglected.

Endophenotypes or intermediate phenotypes are discrete, genetically determined, variables that present at the subclinical level between genotype (genetic vulnerability for disease) and phenotype (clinical entity of disease). They may be indicative of underlying pathogenic processes. Endophenotypes are not discernible by clinical observation. Their identification requires objective quantitative methods (e.g. laboratory-based tests). As trait markers they exist independently, irrespective of the manifestation of disease.

The aim of this thesis was to examine two potential peripheral biological endophenotypes in psychotic disorder, namely cumulative estrogen exposure (reflected by bone mineral density (BMD)) and serum $\mathrm{S} 100 \mathrm{~B}$, in relation to structural cerebral measures. This was done by comparing both patients with psychotic disorder and their non-psychotic siblings to controls. To validate these proxy markers as indicators of cerebral status, associations between each peripheral marker and indices of cerebral structure (i.e. grey and white matter status represented by cortical thickness (CT) and fractional anisotropy (FA) respectively) were assessed.

In chapter 2, bone mineral density (BMD) was used as an indicator of lifetime estrogen exposure to elucidate alterations in its neuroprotective effects in relation to varying levels of genetic risk of psychotic disorder. We found that familial risk of psychotic disorder was not associated with BMD. Therefore, reduced BMD is not an endophenotype of psychotic disorder. Femoral BMD was decreased in female patients, potentially reflecting disease effects and non-familial risk associated with low cumulative estrogen exposure.

In chapter 3, the association between cumulative (lifetime) estrogen exposure and cerebral structural measures ( $C T$ and FA) was explored in female patients. There was no evidence of association between lifetime estrogen exposure and white matter integrity (represented by FA). However, higher cumulative exposure to estrogen was associated with increased $\mathrm{CT}$ in women with psychotic disorder, indicative of the neuroprotective effects of estrogen. 
After a follow-up period of 3 years, BMD was reassessed in the original sample (chapter 4). There was no evidence of continuous low estrogen levels in psychotic disorder, as there was no disproportionate BMD loss over a 3-year period in patients using AP medication. Optimistically, our findings may be reassuring for patients who take prolactin-raising AP. However, given the scarcity of longitudinal work and inconsistencies in outcome, it deserves recommendation to remain aware of sequelae of hyperprolactinemia such as hypogonadism and BMD loss, and to assess side-effects of prolactinraising AP in each individual patient.

For the first time, S100B was investigated as an endophenotype of psychotic disorder in the study delineated in chapter 5. We inspected whether S100B levels were associated with (familial risk of) psychotic disorder in two large and independent samples. Serum S100B levels in patients and siblings were not different from controls. The absence of S100B elevation in (relatively stable) patients and their siblings in our study, in combination with previously reported positive findings in predominantly hospitalized patients (suggesting higher levels of symptomatology), suggests that S100B elevations may reflect fluctuating disease processes in certain patient populations, i.e. it is "state" dependent.

To date, the validity of $\mathrm{S100B}$ as a proxy marker of grey and white matter status has not been established in psychotic disorder. Furthermore, it remains unclear whether S100B exhibits neurotrophic or neurotoxic effects in individuals affected by psychosis. We studied the potential association between serum S100B and neuroimaging markers in patients with a psychotic disorder, their non-psychotic siblings and controls, in order to assess S100B-associated altered brain structure in the context of increased risk (patients and siblings) and disease-effects (patients) of psychotic disorder (chapter 6). No evidence was present for S100B as a proxy marker of grey or white matter status in healthy individuals or patients with psychotic disorder. As in our first S100B study that found no association between (risk of) psychotic disorder and S100B, we found no associations between S100B and grey or white matter markers in health or psychotic disorder.

Biomarkers may complement endophenotypes as indicators of vulnerability. In clinical practice, valid biomarkers assist in the diagnostic process and enable monitoring of disease course and treatment response. At present, no biomarkers have been identified that are specific to psychotic disorder or its prodromal stage. Their identification may offer insight into etiological mechanisms of disease and offer new treatment perspectives.

Currently, psychiatric diagnoses are based on clinical observation and course of symptoms. In practice this can be an extended process that requires time while time is an important determinant in prognosis. Fast and correct diagnosis is paramount. An etiological diagnosis would be superior, more reliable with less room for error and may enable individually tailored treatment and prediction of outcome. 



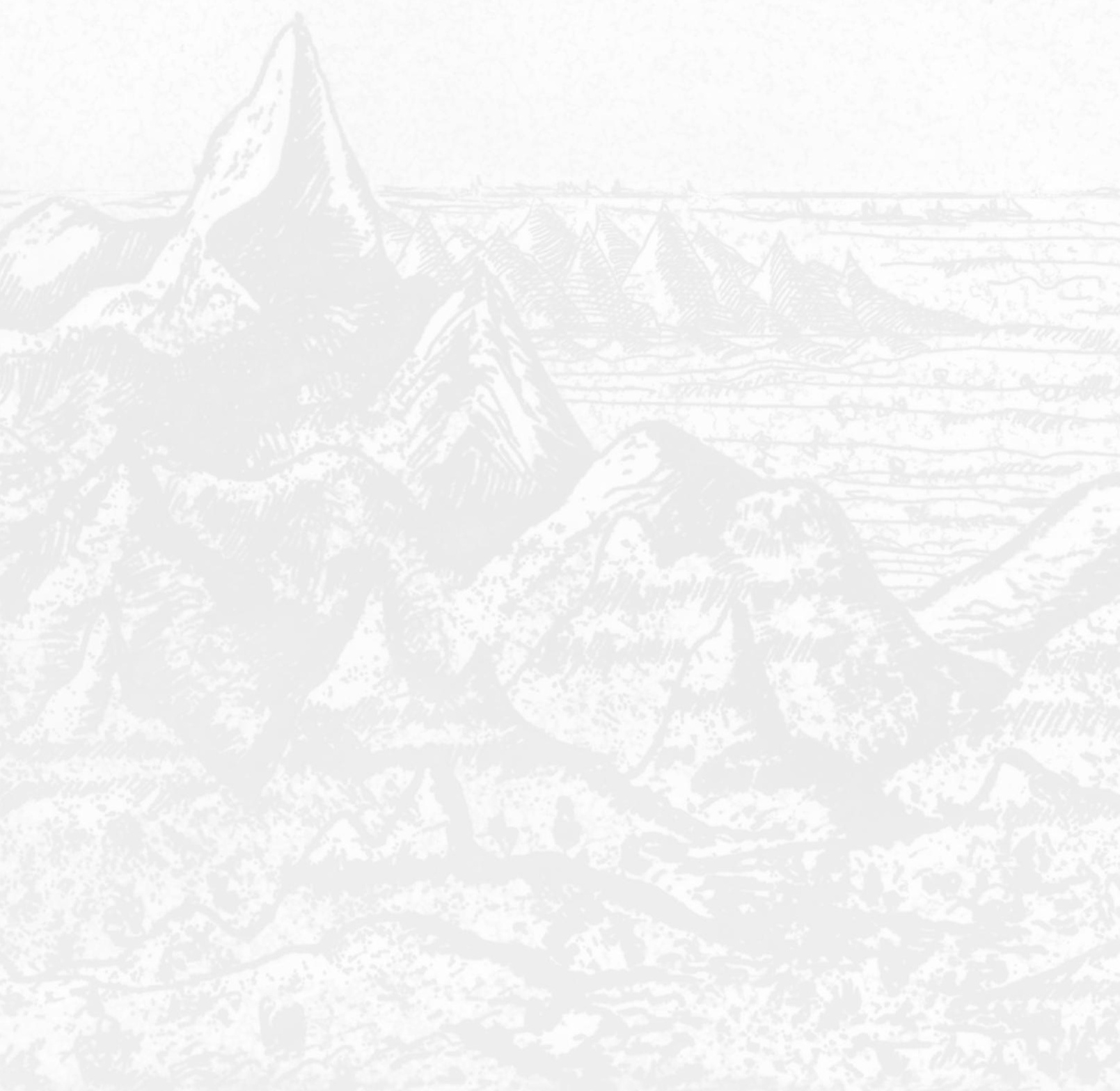


Nine

Samenvatting 

Psychotische stoornissen, waarvan schizofrenie wellicht de meest bekende is, zijn gekenmerkt door de aanwezigheid van zintuiglijke waarnemingen zonder externe stimuli (hallucinaties) en/of gestoorde denkinhoud (wanen). De realiteitstoetsing is niet langer intact. De prevalentie van schizofrenie bedraagt circa 1\%. De etiologie is veelal onbekend. Erfelijkheids- en omgevingsfactoren interacteren en komen samen op een "final common pathway" waarop toegenomen dopaminerge activiteit in het mesolimbisch systeem leidt tot hallucinaties en wanen.

Vele biologische mechanismen beïnvloeden of worden beïnvloed door dopamine, waarbij de rol van andere neutrotransmitters niet genegeerd moet worden.

Endofenotypen of intermediaire fenotypen zijn discrete, genetisch bepaalde, variabelen die aanwezig zijn op subklinisch niveau tussen genotype (erfelijke kwetsbaarheid voor ziekte) en fenotype (klinisch vastgestelde ziekte). Ze duiden mogelijk op onderliggende pathologische processen. Endofenotypen zijn niet door klinische observatie waarneembaar. Het aantonen van endofenotypen vereist objectieve kwantitatieve methoden (bijvoorbeeld door middel van laboratoriumbepaling). Als "trait" marker zijn zij constant, ongeacht de aanwezigheid van ziekte.

Het doel van de studies in dit proefschrift was om twee potentiële endofenotypen van psychotische stoornis te onderzoeken, namelijk cumulatieve oestrogeenblootstelling (weergegeven door minerale botdichtheid (bone mineral density, BMD)) en het eiwit S100B in serum, in relatie tot structurele breinmaten. Dit werd uitgevoerd door patiënten met een psychotische stoornis en hun nietaangedane broers en zussen te vergelijken met controles. Associaties met indicatoren van grijze stof (corticale dikte) en witte stof (fractionele anisotropie (FA)) werden onderzocht om hun bruikbaarheid als perifere markers voor het brein te toetsen.

In hoofdstuk 2 werd BMD gebruikt als maat voor cumulatieve oestrogeenblootstelling om de mate van neuroprotectie te onderzoeken bij verschillende niveaus van erfelijk risico voor psychotische stoornissen. Familiale belasting voor psychotische stoornissen was niet geassocieerd met BMD en dus is BMD geen endofenotype voor psychotische stoornissen. BMD gemeten in de femur van vrouwelijke patiënten bleek verlaagd, wijzend op zowel effecten van ziekte en behandeling als primaire lage cumulatieve oestrogeenblootstelling.

In hoofdstuk 3 werd het verband tussen cumulatieve oestrogeenblootstelling (met BMD als indirecte maat) en structurele breinmaten (corticale dikte en FA) onderzocht in vrouwelijke patiënten. Er was geen bewijs voor associatie tussen oestrogeenblootstelling en witte stof integriteit (FA). Echter, hogere BMD (en dus hogere oestrogeenblootstelling) werd geassocieerd met grotere corticale dikte in vrouwen met een psychotische stoornis, mogelijk representatief voor de neuroprotectieve effecten van oestrogenen. 
Na drie jaar werd BMD opnieuw bepaald in hetzelfde sample (hoofdstuk 4). Er werd geen bewijs gevonden voor continue lage oestrogeenblootstelling, aangezien BMD niet disproportioneel was gedaald in patiënten met psychotische stoornis die antipsychotica (AP) gebruikten. In het meest gunstige geval, zijn deze bevindingen geruststellend voor patiënten die prolactine-verhogende AP gebruiken. Echter, gezien het gebrek aan longitudinaal BMD onderzoek en de inconsistente bevindingen in de spaarzame follow-up studies die tot heden zijn uitgevoerd, is het aan te raden alert te blijven op iatrogene gevolgen van prolactine-verhogende antipsychotica zoals hypogonadisme en osteoporose in elke individuele patiënt.

S100B werd voor het eerst onderzocht als endofenotype voor psychotische stoornissen in de studie beschreven in hoofdstuk 5. Het verband tussen serum S100B en (familiair risico op) psychotische stoornissen werd onderzocht in twee grote, onafhankelijke samples. Serum S100B waarden bleken niet verhoogd in patiënten en hun broers en zussen vergeleken met controles. Het feit dat S100B niet verhoogd is gebleken in de relatief stabiele patiënten in onze studie, gecombineerd met eerder beschreven S100B verhoging in klinische patiënten in de literatuur, suggereert dat fluctuerende ziekteprocessen ten grondslag liggen aan S100B verhoging in bepaalde patiëntenpopulaties.

Tot op heden is de validiteit van S100B als perifere marker voor grijze en witte stof niet aangetoond bij psychotische stoornissen. Daarnaast is het vooralsnog niet opgehelderd of S100B neurotrofe of neurotoxische effecten uitoefent bij psychose. Het verband tussen serum S100B en structurele breinmaten (corticale dikte en FA) werd onderzocht zoals beschreven in hoofdstuk 6. Patiënten, hun gezonde broers/zussen en controles werden vergeleken om onderscheid te maken tussen S100Bgeassocieerde afwijking in breinmaten ten gevolge van verhoogd risico (patiënten en broers/zussen) en effecten van ziekte (patiënten). Er was geen bewijs voor de validiteit van serum S100B als proxy marker voor de breinmaten corticale dikte en FA in patiënten met een psychotische stoornis, hun broers/zussen, noch gezonde controles. Deze bevindingen liggen in dezelfde lijn als die van de eerste S100B studie (hoofdstuk 5), die evenmin verschillen aantoonde tussen de drie groepen.

Biologische markers of biomarkers kunnen endofenotypen aanvullen als indicatoren van kwetsbaarheid voor ziekte. In de klinische praktijk ondersteunen biomarkers diagnostiek en de monitoring van behandeleffecten en beloop. Tot op heden zijn er geen specifieke biomarkers bekend voor psychotische stoornissen of de prodromale fase die aan de stoornis voorafgaat. Het identificeren van biomarkers zou inzicht bieden in de etiologie en de ontwikkeling van nieuwe behandelopties faciliteren. Tot de dag van vandaag worden psychiatrische diagnosen echter gesteld op basis van klinische observatie en het beloop van symptomen. In de praktijk kan dit een tijdsintensief proces zijn terwijl tijd juist een belangrijke determinant is voor prognose. Een snelle en correcte diagnose is van groot belang. Diagnostiek en classificatie van stoornissen op basis van etiologie zou superieur en meer betrouwbaar zijn met een betere individuele behandeling en voorspelling van beloop. 



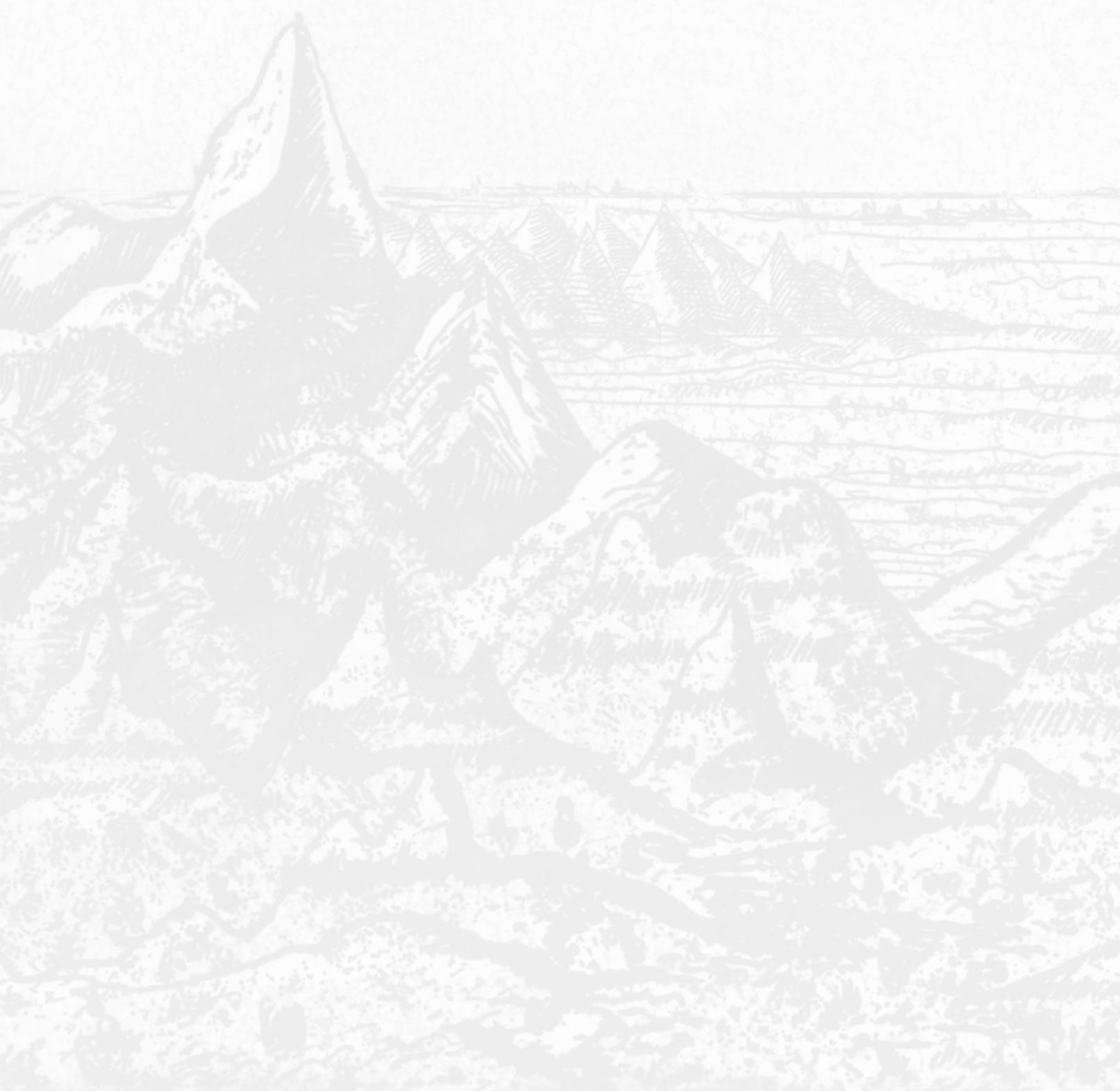




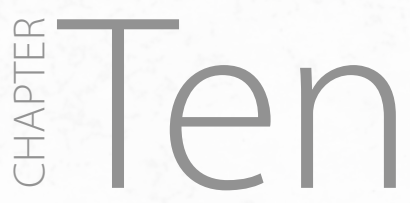

Valorization 

The cost of schizophrenia and other psychotic disorders is high. Not only do patients pay by the disease burden they experience in daily life, their lives are cut short by more than ten years compared to the unaffected population. Increased mortality risk may only be ascribed to suicide for a small part; the better part is due to less access to health care, lifestyle factors and possible iatrogenic effects of treatment, e.g. metabolic syndrome due to second generation antipsychotic medication (Laursen et al., 2013; Saha et al., 2007; van Os and Kapur, 2009). The economic cost for society is accordingly high (Kennedy et al., 2014).

Despite these grim statistics, schizophrenia is not necessarily the debilitating and deteriorating disease it was once thought to be. Prospective studies show that outcome varies greatly a year after diagnosis. Roughly a third of patients show good outcome, another third has poor outcome, with the remaining patients at an intermediate level (van Os and Kapur, 2009). Treatment generally constitutes the prescription of antipsychotic medication combined with application of supportive interventions in the patient's psychological and social context. Generally speaking, pharmacological treatment is still a one-trick pony, i.e. dopamine receptor antagonism. With advancing insights into etiological mechanisms, new treatment strategies will develop and outcome stands to improve with personal gain for patients, as well as their families, and society as a whole.

Optimistically, these developments may also alter the perception of psychotic disorder and mental illness by the general public. Individuals with psychotic disorder or any other mental illness are not to be feared or avoided. It should be understood that they are people struggling with a medical condition no different from a patient with a physical diagnosis.

Insight in etiological mechanisms in psychotic disorder can be gained by investigating endophenotypes. The studies included in this thesis aimed to examine and differentiate between potential peripheral biological endophenotypes and patient-specific characteristics, in relation to structural cerebral measures. Although no endophenotypes were identified, the patient-specific findings described in this thesis can certainly benefit individuals with psychotic disorder.

Our work has provided new evidence for the estrogen hypothesis of schizophrenia with support for both of its aspects, i.e. estrogen deficiency due to gonadal dysfunction and estrogen-mediated neuroprotection providing a relative defense against psychotic disorder. BMD was found to be reduced in female patients and is possibly indicative of estrogen deficiency. Higher cumulative exposure to estrogen was associated with increased cerebral cortical thickness in women with psychotic disorder. The longitudinal data provided no evidence of continuous low estrogen levels (reflected by excessive loss of bone mass) in psychotic disorder. 
Our studies provide a theoretical basis to explain the efficacy of augmentation of estrogen and selective estrogen receptor modulators (SERMs) to antipsychotic treatment. To date, clinical trials were performed on the premises of epidemiological sex differences while studies with a primary biological perspective were lacking.

Reduced BMD due to secondary disease factors (such as lifestyle and iatrogenic effects of PRLraising AP) deserves to be recognized as an important issue. Awareness of increased prevalence of osteoporosis and fracture risk is warranted in the comprehensive treatment of all health concerns in patients with psychotic disorder.

Serum S100B has been heralded as a promising candidate marker in psychotic disorder. However, in our studies, serum S100B was not elevated in individuals with (increased risk of) psychotic disorder in two large independent samples. Nor did we find proof of the validity of S100B as a proxy marker for grey and white matter status in healthy individuals or patients with psychotic disorder. From the literature, it appears that S100B is possibly too sensitive and too indiscriminate to be useful. S100B elevation is perhaps merely indicative of mental activity and/or stress as it is has been shown in physicians on duty, activity which may indeed be stressful but not quite pathological (Gazzolo et al., 2010).

In general, it should be remembered that negative findings make important scientific contributions and are crucial in marking directions for future research. This will ultimately benefit patients, the file drawer effect will not.

The concept of linking peripheral endophenotypes to cerebral structure (or function) is a potentially useful tool for future research. It may be applicable in investigating therapeutic efficacy and identifying regions of interest in the brain in experimental treatment studies, by acquiring neuroimaging data pre- and post-intervention. 


\section{REFERENCES}

Gazzolo, D., Florio, P., Zullino, E., Giovannini, L., Scopesi, F., Bellini, C., Peri, V., Mezzano, P., Petraglia, F., Michetti, F., 2010. $\mathrm{S} 100 \mathrm{~B}$ protein increases in human blood and urine during stressful activity. Clinical chemistry and laboratory medicine : CCLM / FESCC 48, 1363-1365.

Kennedy, J.L., Altar, C.A., Taylor, D.L., Degtiar, I., Hornberger, J.C., 2014. The social and economic burden of treatmentresistant schizophrenia: a systematic literature review. International clinical psychopharmacology 29, 63-76.

Laursen, T.M., Wahlbeck, K., Hallgren, J., Westman, J., Osby, U., Alinaghizadeh, H., Gissler, M., Nordentoft, M., 2013. Life expectancy and death by diseases of the circulatory system in patients with bipolar disorder or schizophrenia in the Nordic countries. PloS one 8, e67133.

Saha, S., Chant, D., McGrath, J., 2007. A systematic review of mortality in schizophrenia: is the differential mortality gap worsening over time? Archives of general psychiatry 64, 1123-1131.

van Os, J., Kapur, S., 2009. Schizophrenia. Lancet 374, 635-645. 


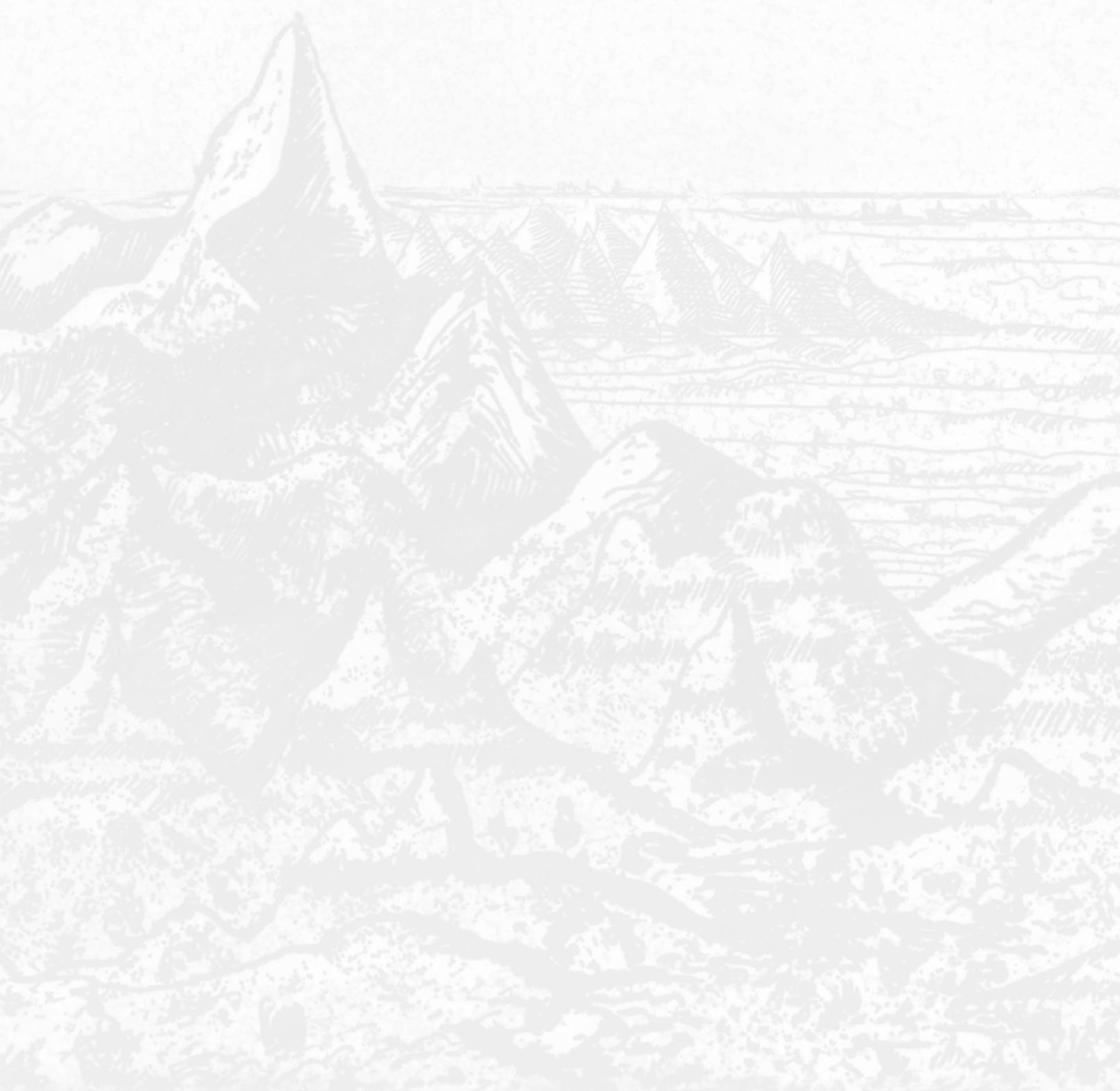


Curriculum vitae

List of publications

Dankwoord 



\section{CURRICULUM VITAE}

Christine van der Leeuw werd geboren op 25 juli 1982. Zij groeide op in Geldrop, Hammondsport (New York, VS) en Waalre.

Na het VWO aan het Sint-Joriscollege te Eindhoven, ging zij geneeskunde studeren aan de Universiteit Maastricht in 2001. Tijdens haar studie bezocht zij voor keuzeonderwijs en coschappen meermaals het buitenland: Engeland (keuzeblok International Health Care Systems), Argentinië (keuzecoschap Neonatologie) en lerland (coschap Neurologie). In 2007 behaalde zij haar artsexamen.

Vervolgens werkte zij als basisarts in een Functional Assertive Community Treatment team van het voormalige PMS Vijverdal te Maastricht. In 2008 begon zij aan de Universitaire Opleiding Psychiatrie Zuid-Limburg. In 2011 ging haar promotietraject van start, waarvoor de specialisatie gedurende een jaar onderbroken werd. Zij voltooide de opleiding tot psychiater in 2013.

Sindsdien is zij als universitair docent verbonden aan de UM. Het eerste jaar als psychiater was zij werkzaam op de spoedzorg van Mondriaan. Sinds februari 2015 werkt zij bij Virenze Riagg Maastricht op de afdelingen stemmings- en angststoornissen en ouderenzorg. 


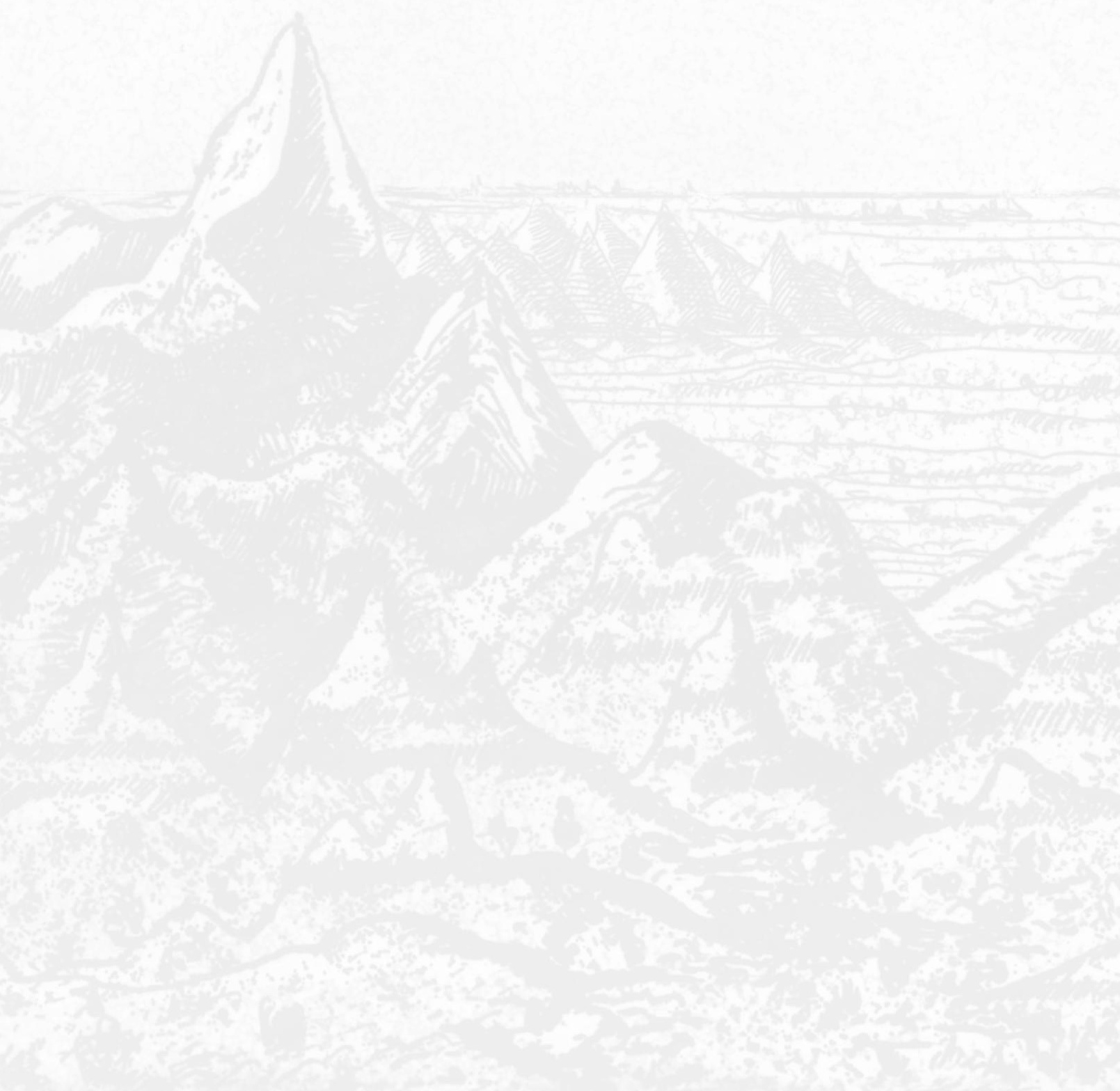




\section{List of publications}





\section{LIST OF PUBLICATIONS}

Van der Leeuw, C., Habets, P., Domen, P., van Kroonenburgh, M., van Os, J., Marcelis, M.; for G.R.O.U.P., 2013. Bone mineral density as a marker of cumulative endogenous estrogen exposure: Relationship to background genetic risk of psychotic disorder. Schizophr Res 143 (1), 25-31.

Van der Leeuw, C., Domen, P., Gronenschild, E., Habets, P., Michielse, S., Van Kroonenburgh, M., Van Os, J., Marcelis, M.; for G.R.O.U.P., 2013. Testing the estrogen hypothesis of schizophrenia: Associations between cumulative estrogen exposure and cerebral structural measures. Schizophr Res 150 (1), 114-120.

Van der Leeuw, C., Marcelis, M., Peeters, S., Verbeek, M., Menheere, P., de Haan, L., van Os, J., van Beveren, N., for G.R.O.U.P., 2013. Replicated evidence of absence of association between serum S100B and (risk of) psychotic disorder. PLoS One 8(12), e82535.

Van der Leeuw, C., Peeters, S., Domen, P., van Kroonenburgh, M., van Os, J., Marcelis, M.; for G.R.O.U.P., 2015. Bone mineral density as a marker of cumulative estrogen exposure in psychotic disorder: a 3 year follow-up study. Submitted.

Van der Leeuw, C., Peeters, S., Gronenschild, E., Michielse, S., Verbeek, M., Menheere, P., van Os, J., Marcelis, M.; for G.R.O.U.P., 2015. Serum S100B: a proxy marker for grey and white matter status in the absence and presence of (increased risk of) psychotic disorder? Submitted. 


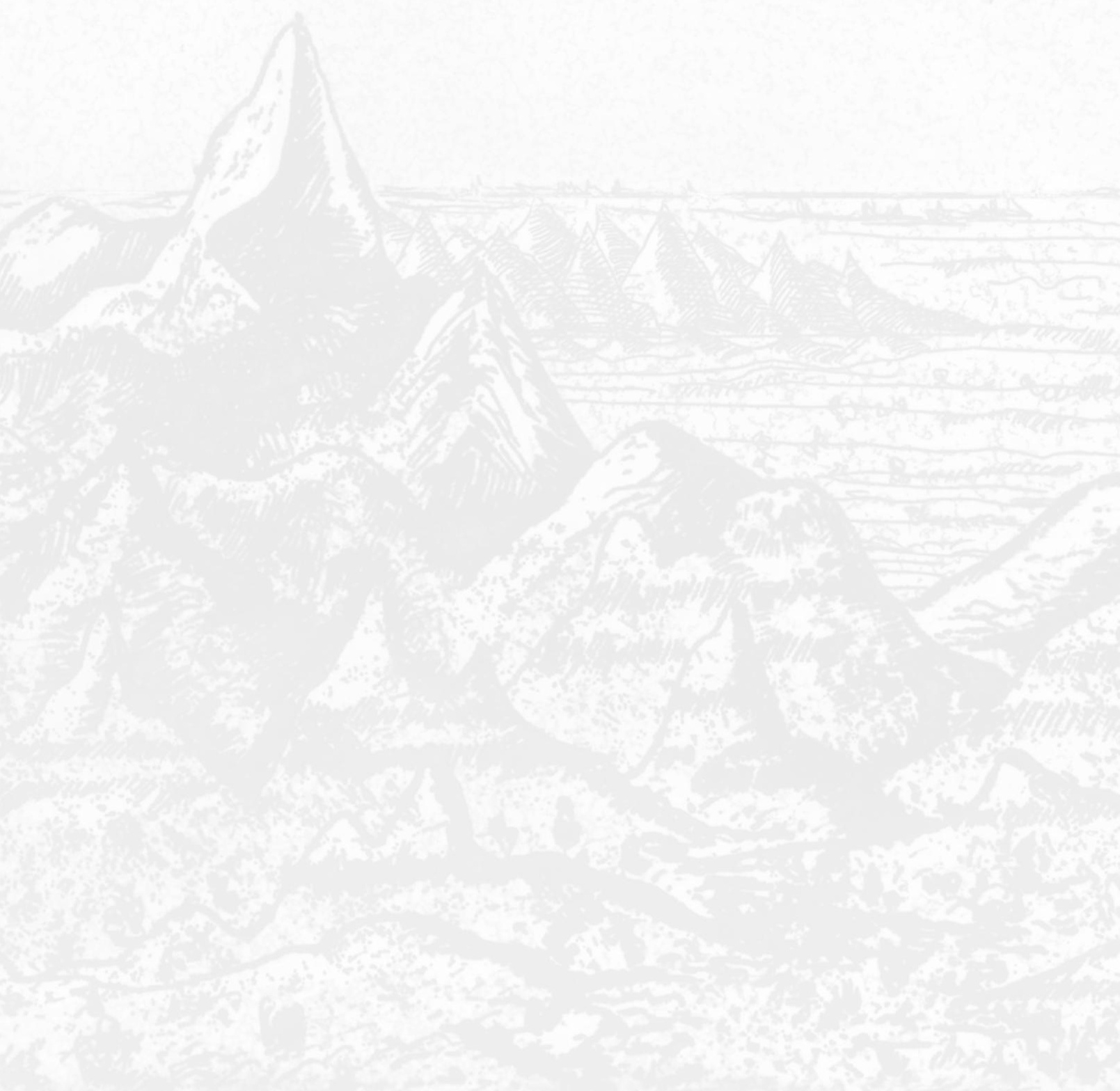


Curriculum vitae

List of publications

Dankwoord 



\section{DANKWOORD}

Jim en Machteld, ik waardeer de samenwerking, begeleiding en alle geboden kansen zeer.

Daarnaast gaat mijn dank uit naar:

Alle lieve collega's aan de universiteit, in het bijzonder de huisgenootjes in Florence: Sanne, Martine, Mayke, Aleida, Feikje \& Dennis. Kamergenoten Ger \& Nicole. Alle co-auteurs, met name "de vaste": Sanne, Patrick, Petra, Ed \& Stijn. Truda, len, Jolanda en Trees: voor jullie geduldige behulpzaamheid.

Oud-collega's Mondriaan, vooral van de spoedzorg natuurlijk. Alle psychiaters, arts-assistenten, verpleegkundigen en secretaresses, teveel om allemaal bij naam te noemen. Collega's Riagg, bedankt voor het warme welkom!

Irene \& Marlous, met jullie is het altijd véél te gezellig. Ook met jullie grote en kleine mannen: Sander, Timon, Steve \& Robin.

Marijke. Samen zijn we heel goed in klagen, veel eten en wijn drinken. Zo hou je me al sinds dag 1 geneeskunde op de been ;-)

Marian, ik mis de heilige woensdagavond nog steeds...

Marlou, onze vriendschap is mijn oudste, op naar de volgende 20 jaar!

Jesse, Pauline, Bart, Simone, Marianne \& Rachel, voor alle gezelligheid en fietstochten.

Dank je Mamma, voor je onvoorwaardelijk vertrouwen in mijn kunnen.

Lisa \& Wilbert, laten we altijd dezelfde sterke verhalen blijven vertellen en elke kerst Home Alone kijken.

En Rein, ik hou van ons. 
